Aus der Poliklinik für Präventive Zahnmedizin, Parodontologie und Kariologie

(Prof. Dr. med. dent. A. Wiegand)

im Zentrum für Zahn-, Mund- und Kieferheilkunde

der Medizinischen Fakultät der Universität Göttingen

\title{
Die Auswirkungen der
}

zahnärztlichen Behandlung in

Intubationsnarkose auf die

mundgesundheitsbezogene

Lebensqualität von Patienten mit

kognitiver Einschränkung

\author{
INAUGURAL-DISSERTATION \\ zur Erlangung des Doktorgrades \\ für Zahnheilkunde \\ der Medizinischen Fakultät der \\ Georg-August-Universität zu Göttingen
}

vorgelegt von

Anna-Lena Hillebrecht

aus

Kassel

Göttingen 2019 
Dekan:

\section{Betreuungsausschuss}

Betreuerin:

Ko-Betreuerin:

\section{Prüfungskommission}

Referent/in:

Ko-Referent/in:

Drittreferent/in:

Datum der mündlichen Prüfung:
Prof. Dr. rer. nat. H. K. Kroemer

Prof. Dr. med. dent. A. Wiegand

Prof. Dr. med. I. Zerr

Prof. Dr. med. dent. A. Wiegand

Prof. Dr. med. I. Zerr

Prof. Dr. Th. Meyer

26.03.2020 
Hiermit erkläre ich, die Dissertation mit dem Titel „Die Auswirkungen der zahnärztlichen Behandlung in Intubationsnarkose auf die mundgesundheitsbezogene Lebensqualität von Patienten mit kognitiver Einschränkung" eigenständig angefertigt und keine anderen als die von mir angegebenen Quellen und Hilfsmittel verwendet zu haben.

Göttingen, den 17.07.2019

(Anna-Lena Hillebrecht) 


\section{Inhaltsverzeichnis}

Abbildungsverzeichnis ........................................................................... III

Tabellenverzeichnis ..................................................................................

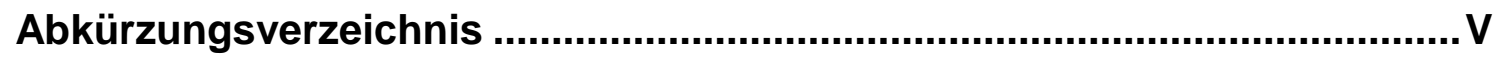

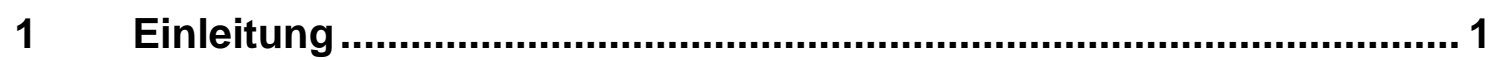

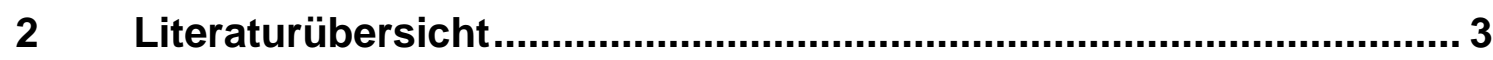

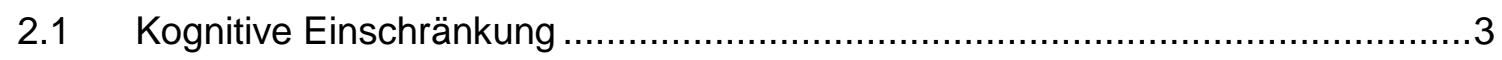

2.2 Ursachen für eine reduzierte Mundgesundheit bei Personen mit kognitiver Einschränkung ...............................................................................

2.3 Mundgesundheitszustand von Personen mit kognitiver Einschränkung ..............8

2.4 Zahnärztliche Therapie von Personen mit kognitiver Einschränkung ….............10

2.5 Mundgesundheitsbezogene Lebensqualität (MLQ) .....................................13

2.6 Oral Health Impact Profile (OHIP) .........................................................17

2.7 Mundgesundheitsbezogene Lebensqualität von Patienten mit kognitiver

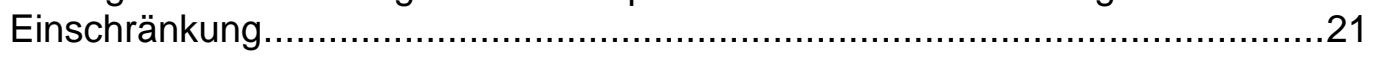

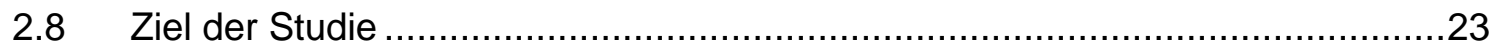

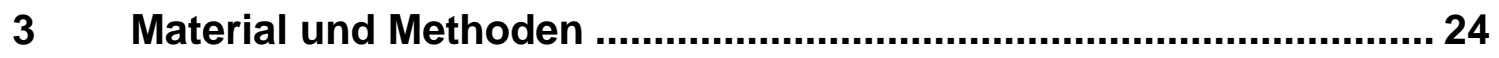

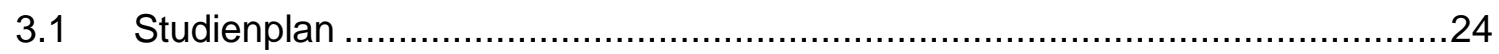

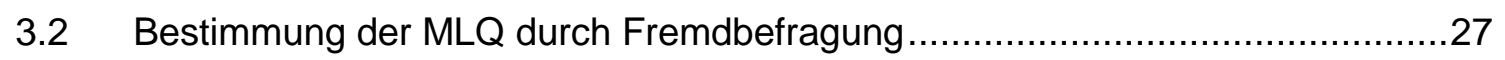

3.3 Bestimmung der MLQ durch Selbstbefragung ...............................................29

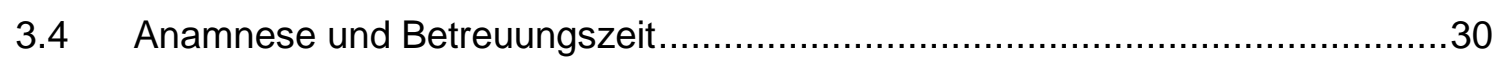

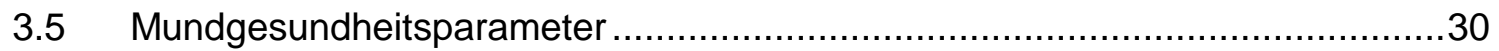

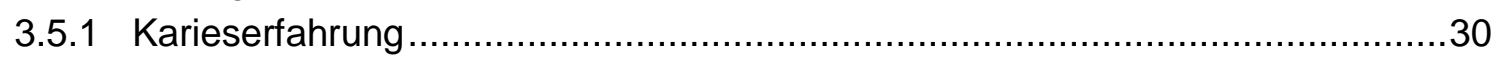

3.5.2 Parodontale Schädigung ………………………..................................

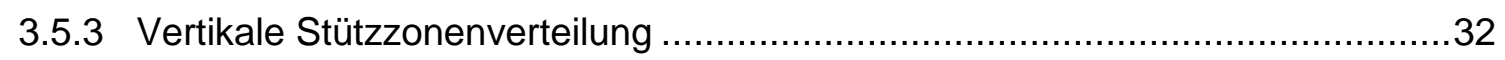

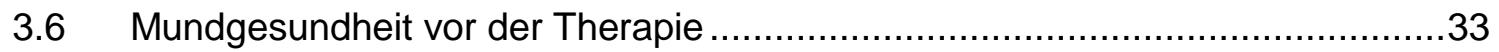

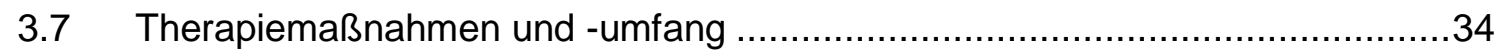

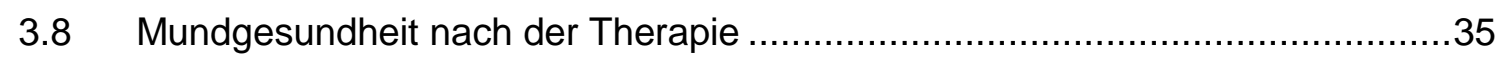

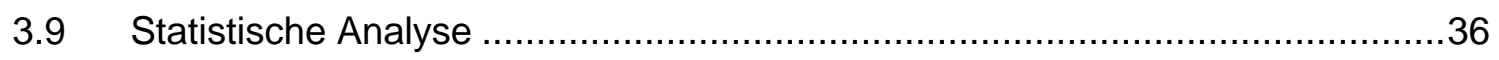

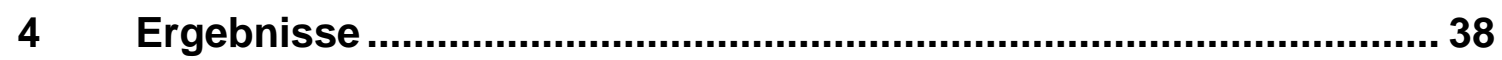

4.1 Mundgesundheitsbezogene Lebensqualität.................................................39

4.1.1 Antwortenverteilung der Fremdbefragung ..................................................

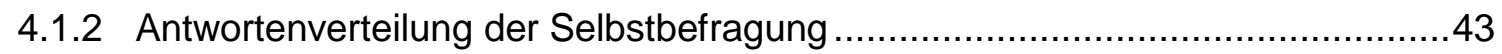

4.2 Korrelation von Selbst- und Fremdbefragung ………....................................

4.3 Patientenkollektiv .................................................................................. 
$4.4 \quad$ Mundgesundheit vor und nach der Therapie ................................................45

4.5 Umfang der zahnmedizinischen Therapiemaßnahmen in ITN ........................48

4.6 Signifikante Einflussfaktoren auf die MLQ vor der Therapie.............................49

4.7 Signifikante Einflussfaktoren auf die MLQ im Verlauf der Studie.......................50

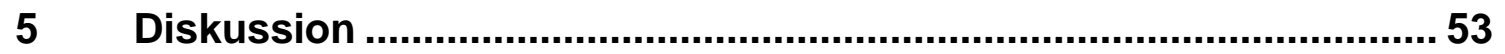

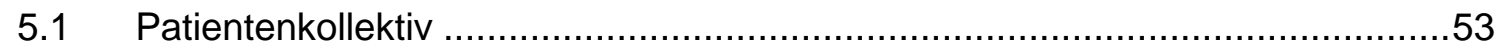

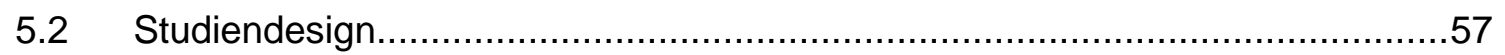

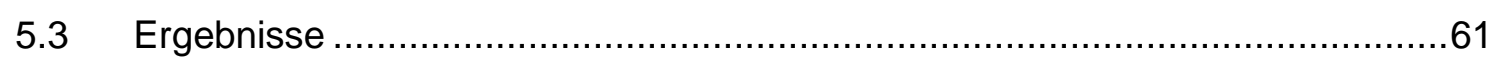

5.3.1 Bewertung der MLQ durch die Fremdbefragung .........................................61

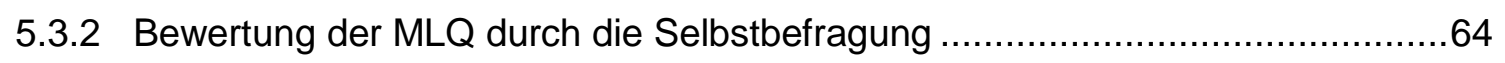

5.3.3 Korrelation von Betreuer- und Patientenbefragung ....................................66

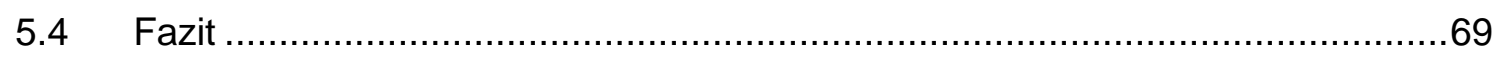

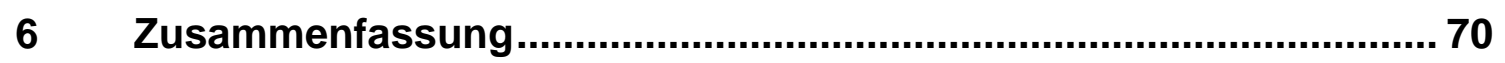

$7 \quad$ Literaturverzeichnis .................................................................. 71 


\section{Abbildungsverzeichnis}

Abbildung 2-1: Intraorale Fotoaufnahme eines männlichen Patienten mit angeborener Behinderung unklarer Genese

Abbildung 2-2: Mundgesundheitsbezogene Lebensqualität als Teilbereich der gesundheitsbezogenen und allgemeinen Lebensqualität nach John und Micheelis.....13

Abbildung 2-3: Theoretisches Modell zur MLQ nach Locker ......................................15

Abbildung 2-4: Theoretisches Modell zur MLQ nach Sischo und Border.....................16

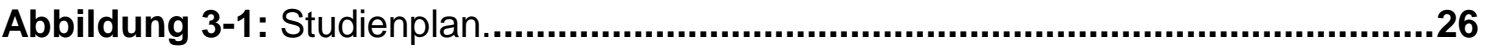

Abbildung 4-1: Studienablauf und Anzahl teilnehmender Patienten bzw. Betreuer ....39

Abbildung 4-2: Summenwerte des OHIP-G14 ........................................................ 40

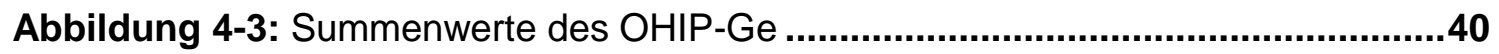

Abbildung 4-4: Summenwerte des OHIP-G14 von Patienten mit Selbstbefragung und Patienten ohne Selbstbefragung ...................................................................................... 41 


\section{Tabellenverzeichnis}

Tabelle 2-1: Zahnmedizinische funktionelle Kapazität..................................................12

Tabelle 2-2: Oral-Health-Impact-Profile-German-14 (OHIP-G14)................................19

Tabelle 2-3: Oral Health-Impact-Profile-German-5 (OHIP-G5) ....................................20

Tabelle 3-1: Angepasster OHIP-G14-Fragebogen zur Fremdbefragung der

Betreuungspersonen.

Tabelle 3-2: Angepasster OHIP-G5-Fragebogen zur Selbstbefragung der Patienten in Leichter Sprache.

Tabelle 3-3: Erfassung potenzieller Einflussfaktoren auf die MLQ aus der Anamnese 30

Tabelle 3-4: Parodontaler Screening Index (PSI) ......................................................31

Tabelle 3-5: Vertikale Stützzonenverteilung nach Eichner ...........................................32

Tabelle 3-6: Mundgesundheitsparameter zur Beschreibung des

Mundgesundheitszustandes vor der Therapie

Tabelle 3-7: Zahnmedizinische Therapiemaßnahmen und Therapieumfang

Tabelle 3-8: Mundgesundheitsparameter zur Beschreibung des

Mundgesundheitszustandes nach der Therapie

Tabelle 4-1: Antworten der Fremdbefragung (OHIP-G14).........................................42

Tabelle 4-2: Antworten der Selbtbefragung (OHIP-Ge)..............................................43

Tabelle 4-3: Spearman Korrelationskoeffizienten der Betreuer- und

Patientenbefragung.

Tabelle 4-4: Demografische Merkmale der Patientenklientel

Tabelle 4-5: Karieserfahrung (DMF-T) vor und nach der Therapie in ITN. ..................46

Tabelle 4-6: Zahnanzahl der Patienten vor und nach der Therapie in ITN ...................47

Tabelle 4-7: Vertikale Stützzonenverteilung nach Eichner vor und nach der Therapie.48

Tabelle 4-8: Therapiemaßnahmen während der ITN

Tabelle 4-9: Signifikante Einflussfaktoren auf den OHIP-G14 zu T0

Tabelle 4-10: Signifikante Einflussfaktoren auf die Summenwertänderung des OHIP-

Tabelle 4-11: Signifikante Einflussfaktoren auf die Summenwertänderung des OHIP-

Tabelle 5-1: Vergleich der Ergebnisse zur Karieserfahrung mit anderen nationalen kariesepidemiologischen Studien 


\section{Abkürzungsverzeichnis}

DMF-T Zahnbezogener Kariesindex, Anzahl der kariösen (decayed), fehlenden (missing), gefüllten (filled) permanenten Zähne (teeth)

ITN Intubationsnarkose

MLQ Mundgesundheitsbezogene Lebensqualität

OHRQoL Oral Health Related Quality of Life (Mundgesundheitsbezogene Lebensqualität)

OHIP Oral Health Impact Profile

OHIP-14 Oral Health Impact Profile - englische Version, 14 Fragen

OHIP-G14 Oral Health Impact Profile German - deutsche Version, 14 Fragen

OHIP-Ge Oral Health Impact Profile German easy - deutsche Version in Leichter

Sprache

PSI

Parodontaler Screeningindex

WHO

World Health Organization (Weltgesundheitsorganisation) 


\section{Einleitung}

Patienten mit kognitiven Einschränkungen weisen im Vergleich zur Durchschnittsbevölkerung eine schlechtere Mundhygiene und häufig einen umfangreichen zahnärztlichen Therapiebedarf auf (Pieper et al. 1986; Shaw et al. 1990; Winter et al. 2008; Anders und Davis 2010; Schulte et al. 2011; Schulte et al. 2013; Hempel et al. 2015; Naseem et al. 2016; Kothari et al. 2017). Der hohe Sanierungsbedarf in Kombination mit mangelnder Kooperation macht häufig die zahnmedizinische Rehabilitation im Rahmen einer Vollnarkose (Intubationsnarkose, ITN) erforderlich (Manley et al. 2000; Dougherty 2009; Mallineni und Yiu 2015; Lim und Borromeo 2017; Glassman 2009). Die zahnmedizinischen Maßnahmen im Rahmen der ITN richten sich nach dem individuellen Therapiebedarf des Patienten und beinhalten zumeist präventive (Zahnreinigungen), konservierende (Füllungstherapien) und oralchirurgische Maßnahmen (Zahnextraktionen) (Lim und Borromeo 2017). Um eine erneute Behandlung in ITN zu vermeiden, werden Zähne mit fraglicher Prognose (z. B. Zähne mit Verdacht auf eine Pulpitis oder Pulpanekrose sowie Zähne mit starker parodontaler Schädigung) i. d. R. entfernt. Ziel der Behandlung ist die Schmerzbeseitigung sowie die Wiederherstellung von Mundgesundheit bei maximalem Kaukomfort. Bislang ist nicht bekannt, wie sich diese umfassende zahnmedizinische Rehabilitation auf die Lebensqualität der betroffenen Patienten auswirkt. Das Konzept der mundgesundheitsbezogenen Lebensqualität (MLQ) beschreibt die Auswirkungen von Mundgesundheit auf das Wohlbefinden und die allgemeine Lebensqualität (Cushing et al. 1986; Locker 1988; Allen 2003; Sischo und Broder 2011). Die MLQ kann mittels des Oral Health Impact Profile (OHIP), einem international anerkannten und validierten Fragebogen zur Bestimmung der MLQ von Einzelpersonen oder Patientengruppen, quantifiziert werden (Slade und Spencer 1994; Slade 1997; John et al. 2002; John et al. 2004; John et al. 2006). Der OHIP dokumentiert das Auftreten von Schmerzen sowie soziale, ästhetische und funktionelle Einschränkungen im Zusammenhang mit der Mundgesundheit. Die OHIP-Befragung von Patienten vor und nach zahnärztlicher Intervention hat sich wissenschaftlich als patientenzentrierter Maßstab für den zahnmedizinischen Therapieerfolg etabliert (Allen 2003). In verschiedenen Studien konnte eine Verbesserung der MLQ durch zahnerhaltende, protheti- 
sche oder kieferorthopädische Behandlungen festgestellt werden (Dugas et al. 2002; Heydecke et al. 2003; Szentpétery et al. 2006; Nickenig et al. 2008; Brauchle et al. 2013; Aarabi et al. 2015; Jenei et al. 2015; Kolenda et al. 2016; Mendez et al. 2017). Eine Reduktion der Zahnanzahl wirkte sich dagegen eher negativ auf die MLQ aus (Brennan et al. 2008; Gerritsen et al. 2010; Adeyemo et al. 2012; Bortoluzzi et al. 2012).

Neben zahnmedizinischen Befunden beeinflussen patientengruppenbezogene Faktoren, wie Alter oder Sozialisierung, den individuellen Anspruch und die Bewertung der MLQ (Steele et al. 2004). Um diese subjektiven Faktoren zu erfassen, sollten Patienten die OHIP-Fragen selbstständig beantworten. Eine Befragung über Dritte in Form einer Fremdbefragung wird notwendig, wenn die Patienten, z. B. Kleinkinder, selbst keine oder nur eingeschränkt Aussagen zu ihrer MLQ machen können. Allerdings besteht bei einer Fremdbefragung das Risiko von Fehleinschätzungen (Eiser und Morse 2001; Wilson-Genderson et al. 2007; Lee et al. 2017). Bislang liegen zur MLQ von Patienten mit kognitiver Einschränkung nur wenige Daten vor (Loureiro et al. 2007; Jensen et al. 2008; Chang et al. 2014; Alves Nayara Silva et al. 2016; Ho et al. 2017; Couto et al. 2018), wobei die MLQ der betroffenen Patienten überwiegend über Fremdbefragungen ermittelt wurde (Loureiro et al. 2007; Jensen et al. 2008; Chang et al. 2014; Alves Nayara Silva et al. 2016).

Ziel dieser Untersuchung war es, die MLQ von Patienten mit kognitiver Einschränkung vor und nach zahnmedizinischer Therapie in ITN zu quantifizieren und zu vergleichen. Dazu sollten zum einen - im Hinblick auf einen personenzentrierten Ansatz - die Patienten selbst (sofern möglich) und zum anderen deren Betreuungspersonen befragt werden. Die Übereinstimmung der Bewertung von Patienten und Betreuungsperson sollte ebenfalls überprüft werden. Ferner sollte untersucht werden, ob die MLQ durch behinderungsassoziierte Faktoren, klinische Mundgesundheitsparameter sowie durch die im Rahmen der Narkose erfolgten Therapiemaßnahmen beeinflusst wird. 


\section{Literaturübersicht}

\subsection{Kognitive Einschränkung}

Das menschliche Gehirn verfügt über eine Reihe von kognitiven Fähigkeiten. Diese umfassen die Wahrnehmung von Signalen aus der Umwelt und deren Weiterverarbeitung. Bei einer kognitiven Einschränkung sind diese Fähigkeiten reduziert. Emotionale, körperliche, soziale und kommunikative Fähigkeiten können durch die kognitive Einschränkung in unterschiedlichem Ausmaß betroffen sein (Pörtner 2006; Janz und Terfloth 2009; Hirschberg 2014). Verschiedene theoretische Modelle definieren Standardanforderungen an die kognitiven Fähigkeiten einer Person. Personen mit kognitiver Einschränkung weichen von diesen (meist intellektuellen und gesellschaftlichen) Standards ab, ohne ihren Zustand selbstständig beeinflussen zu können. Die Standardanforderungen an die kognitiven Fähigkeiten einer Person unterscheiden sich je nach Fachdisziplin und Situation. Eine Homogenisierung und Deskription von Personengruppen nach ihrer kognitiven Kondition sollte daher stets entsprechend den fachspezifischen Anforderungen an das intellektuelle Leistungsvermögen erfolgen (Carroll 1993). Die Ursachen für eine kognitive Einschränkung sind nicht immer bekannt. Möglich sind chromosomale, genetische, neurologische Erkrankungen und/oder traumatische Ereignisse (Theunissen et al. 2013). Allgemein lässt sich sagen, dass bei einer relevanten kognitven Einschränkung, unabhängig von der Ätiologie, Diskrepanzen zwischen Anforderungen und Leistungsfähigkeit einer Person bestehen. Durch eine kognitive Einschränkung ist die Fähigkeit, neue oder komplexe Informationen zu verstehen und neue Fähigkeiten zu erlernen bzw. anzuwenden im Vergleich zur Durchschnittsbevölkerung reduziert (beeinträchtigte Intelligenz). Dadurch verringert sich die Fähigkeit, ein unabhängiges Leben zu führen (beeinträchtigte soziale Kompetenz). Eine kognitive Einschränkung ist kein isoliertes Krankheitsbild, sie kann allerdings direkte Auswirkungen auf die Gesundheit (körperliche Integrität) der betroffenen Person haben. In der UN-Behintertenrechtskonvention wird die Gruppe von Menschen mit Behinderung definiert: 
“Menschen die langfristige körperliche, seelische, geistige oder Sinnesbeeinträchtigungen haben, welche sie in Wechselwirkung mit verschiedenen Barrieren an der vollen, wirksamen und gleichberechtigten Teilhabe an der Gesellschaft hindern können." (Artikel 1, Satz 2 der UN-Behindertenrechtskonvention)

Die aktuelle Definition der Weltgesundheitsorganisation (WHO) bezieht neben der medizinischen Definition (beeinträchtigte Intelligenz: jemand ist behindert) auch gesellschaftliche Komponenten mit ein (Rahmenbedingungen: jemand wird behindert) (WHO 2011). Ob und in welchem Ausmaß aus der kognitiven Einschränkung eine Behinderung resultiert, wird folglich von dem kompensatorischen Verhalten des sozialen Umfeldes mit beeinflusst. Umweltbedingte Faktoren können die Alltagsaktivitäten für Personen mit kognitiver Einschränkung erleichtern oder erschweren (Verbrugge und Jette 1994). In vielen Bereichen des öffentlichen Interesses hat ein Anpassen von Informationsdokumenten für Personen mit reduzierter kognitiver Leistungsfähigkeit stattgefunden. Beispielsweise werden Nachrichten und Gesetzestexte in eine vereinfachte Sprache (Leichte Sprache) übersetzt. Die Leichte Sprache unterliegt festen Regeln. Komplizierte Formulierungen werden dabei vermieden und Inhalte verständlich präsentiert, so dass Informationen auch für Personen mit reduzierter kognitiver Leistungsfähigkeit zugänglich sind (Winter 2014; Bredel und Maass 2016).

Personen, die aufgrund ihrer kognitiven Einschränkung bei der Gestaltung ihres Altages auf Hilfe angewiesen sind, haben in Deutschland nach §1896 BGB ein Recht auf individuelle Hilfestellungen durch einen Betreuer (gesetzliche Vertretung). Diese gesetzliche Vertretung wird vom Betreuungsgericht für verschiedene Teilbereiche festgelegt. Kann eine Person aufgrund einer reduzierten kognitiven Leistungsfähigkeit die eigene Gesundheitsfürsorge nicht realitäts- oder sachgerecht erledigen und die körperliche Integrität nicht aufrechterhalten, ist es Aufgabe des gesetzlichen Vertreters für die Umsetzung einer adäquaten Gesundheitsfürsorge des Betreuten zu sorgen (§ 1896 BGB Voraussetzungen). Die UN-Behindertenrechtskonvention (2009) liefert darüber hinaus die Rechtsgrundlage für ein Höchstmaß an Gesundheit ohne Diskriminierung aufgrund von Behinderung: 
„Insbesondere bieten die Vertragsstaaten die Gesundheitsleistungen an, die von Menschen mit Behinderungen speziell wegen ihrer Behinderung benötigt werden, soweit angebracht, einschließlich Früherkennung und Frühintervention sowie Leistungen, durch die, auch bei Kindern und älteren Menschen, weitere Behinderungen möglichst gering gehalten oder vermieden werden sollen." (Artikel 25 der UN-Behindertenrechtskonvention)

Durch den allgemeinmedizinischen Fortschritt steigt die Lebenserwartung auch von Menschen mit angeborenen oder früh erworbenen kognitiven Einschränkungen. Hinzu kommt eine Zunahme von Personen, deren kognitive Leistungsfähigkeit (meist im höheren Lebensalter) aufgrund neurodegenerativer Erkrankungen abnimmt. In der Gesamtbevölkerung ist mit einer steigenden Anzahl an Personen zu rechnen, die aufgrund ihrer kognitiven Einschränkung spezielle Gesundheitsleistungen benötigen (Manton 1982; Mayer 2013; Nowossadeck 2013).

\subsection{Ursachen für eine reduzierte Mundgesundheit bei Personen mit kognitiver Einschränkung}

Die Mundgesundheit ist ein wesentlicher Bestandteil der Allgemeingesundheit. Der Weltverband der Zahnärzte (FDI, Federation Dentaire Internationale) definierte 2016 den Begriff der Mundgesundheit:

„Mundgesundheit ist vielgestaltig und beinhaltet die Fähigkeit, zu kauen, zu sprechen, zu lächeln, zu riechen, zu schmecken, zu berühren, zu schlucken und Emotionen über Gesichtsausdrücke mit Selbstvertrauen und ohne Schmerz oder Unbehagen sowie ohne Krankheit des kraniofazialen Komplexes zu übermitteln. " (Definition zur Mundgesundheit der FDI 2016, S.2)

Behinderungsassoziierte Faktoren können dazu führen, dass Personen mit kognitiver Einschränkung nicht kauen, nicht sprechen, nicht lächeln, nicht berühren und/oder nicht schlucken können. Auch das Vermitteln von Emotionen über Gesichtsausdrücke ist einigen dieser Personen nicht möglich. Zusätzlich stellen Menschen mit Behinderung laut Bundeszahnärztekammer (BZÄK) eine Hochrisikogruppe für Karies und Parodontitis dar (BZÄK 2017). Die Ursachen für Karies und Parodontitis sind-unabhängig von der Personengruppe - 
multifaktoriell (Carlos und Gittelsohn 1965; Petersen 1990; Sheiham und Nicolau 2005; Sisson 2007). Bei Personen mit kognitiver Einschränkung werden die allgemeinen Risikofaktoren für Karies und Parodontitis durch Folgen oder Begleiterscheinungen der Behinderung modelliert. Im folgenden Abschnitt werden häufig auftretende mundgesundheitsbezogene Krankheitsbilder bei Menschen mit kognitiver Einschränkung und deren behinderungsassoziierten Ursachen zusammengefasst:

Ist eine Hirnschädigung ursächlich für die reduzierte kognitive Leistungsfähigkeit, hat dies meist auch körperliche Folgen. Eine Hirnschädigung im Bereich des motorischen Kortex oder der Pyramidenbahn führt zu zerebralen Bewegungsstörungen und somit zu einem pathologisch veränderten Muskeltonus (Pinel und Pauli 2012). Besteht der pathologische Muskeltonus bereits während der Zahn- und Kieferentwicklung, führt dies zu skelettalen Wachstumsstörungen und dentalen Fehlstellungen (Pommerenke et al. 1988; Kiliaridis 1995; Thilander 1995; Winter et al. 2008; Vellappally et al. 2014). Bei Kindern mit einem reduzierten Muskeltonus und permanenter Mundatmung entwickeln sich die Oberkieferzähne eher protrudiert. Bei stark protrudierten Frontzähnen resultiert ein inkompletter Lippenschluss. Die dauerhafte Mundöffnung führt zu einem Austrocknen der Mundschleimhäute, was wiederum das Risiko für Gingivitis und Parodontitis erhöht (Schopf 1994; Mizutani et al. 2015). Bei protrudierten Oberkieferfrontzähnen besteht außerdem ein erhöhtes Risiko für dentale Traumata (Corrêa-Faria et al. 2016). Generell ist bei einer eingeschränkten Motorik und Selbstwahrnehmung von einem erhöhten Risiko für dentale Verletzungen durch eine erhöhte Sturz- und Selbstverletzungsgefahr auszugehen (Bagattoni et al. 2017). Werden die verletzten Zähne nicht zahnärztlich behandelt, besteht die akute Gefahr von Karies und anderen Infektionen. Aus einem behinderungsbedingt reduzierten Tonus der Speiseröhrenmuskulatur kann bei Patienten, die bei genereller Muskelhypotonie keine aufrechte Körperposition einnehmen können, ein oropharyngealer Reflux resultieren (Mazzoleni et al. 1991; Braegger 1998; Vandenplas und Hegar 2000; Bozkurt et al. 2004). Der Rückfluss von angedauter Nahrung und Magensäure in die Mundhöhle bewirkt neben der Zunahme des Kariesrisikos auch einen Riskoanstieg für einen säurebedingten Zahnhartsubstanzverlust (Erosionen). Ein reduzierter Tonus der Wangen- und Zungenmuskulatur führt zu einer verlängerten Persistenz von 
Nahrungsresten im Vestibulum. Ist ein physiologisches Kauen aufgrund eines oralen Hypotonus nicht möglich, erfolgt die Ernährung überwiegend durch weiche Kost. Um den erforderlichen Kalorienbedarf bei erschwerter Nahrungsaufnahme zu decken, werden einigen Personen vermehrt gesüßte und hochkariogene Lebensmittel angeboten. Die kariogene Ernährung, die verlängerte Retention von Nahrung und ein Reflux von Mageninhalt in die Mundhöhle erhöhen das Risiko für Karies (Idaira et al. 2008).

Bei Kindern mit spastisch erhöhtem Muskeltonus entwickelt sich die knöcherne Basis der Oberkiefer ebenfalls nicht physiologisch. Durch den Muskeldruck auf den Kiefer resultiert ein eher hoher, schmaler Gaumen. In Kombination mit einem dentalen Platzmangel (Zahnengstand) ist die Zahnpflege erschwert und die Retention von Plaque und Nahrungsresten begünstigt (Abbildung 2-1).
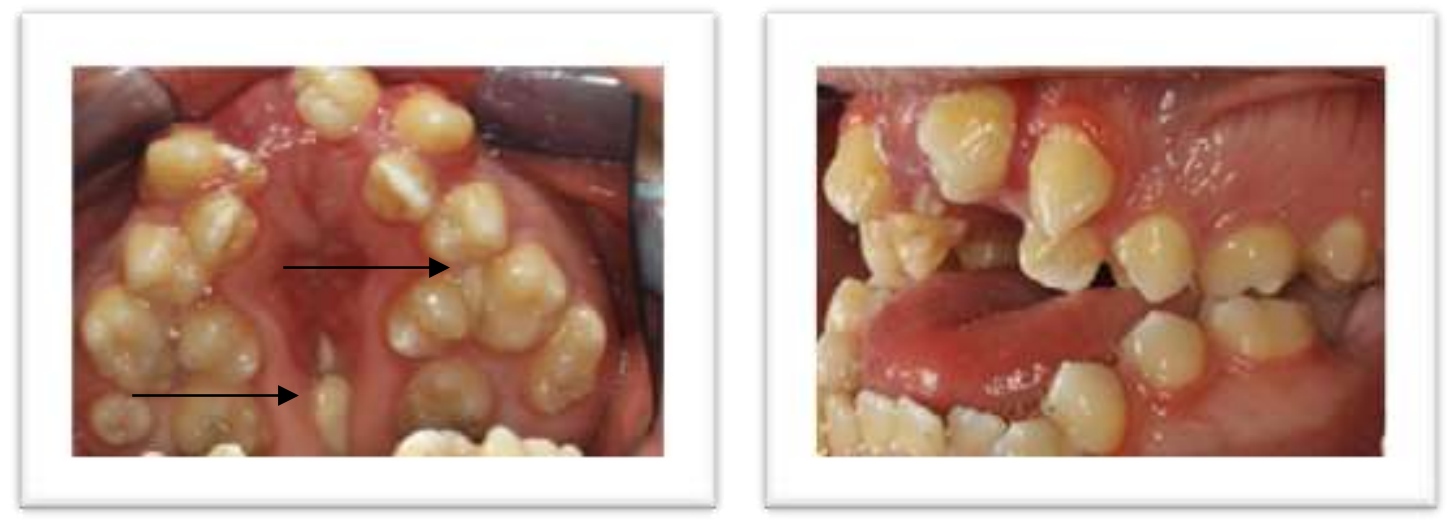

Abbildung 2-1: Intraorale Fotoaufnahme eines männlichen Patienten mit angeborener Behinderung unklarer Genese. Zahnengstand und ein schmaler, hoher Gaumen begünstigen die Plaqueretention. Quelle: Poliklinik für Präventive Zahnmedizin, Parodontologie und Kariologie, Göttingen

Eine mögliche Ursache für eine Schädigung der Hirnsubstanz stellt der Untergang von Nervenzellen durch eine Hypoxie im Rahmen eines epileptischen Krampfanfalles dar. Eine Epilepsie kann auch in Folge einer Hirnschädigung, unabhängig von deren Genese, auftreten. Die Therapie zur Vermeidung erneuter Krampfanfälle erfolgt mit Antikonvulsiva. Bei einigen dieser Medikamente (z. B. Orfiril) werden Gingivahyperplasien als unerwünschte Nebenwirkung beschrieben (Bickley 1978). Diese medikamenteninduzierten Parodontopathien erschweren die Durchführung einer adäquaten Zahnpflege. Bei Patienten mit Trisomie 21 besteht durch eine veränderte Immunreaktion eine genetische Dis- 
position für Parodontitits (Cutando-Soriano et al. 1998; Neuhäuser 2010; Frydman und Nowzari 2012; Molina-García et al. 2016; Scalioni et al. 2018). Zusätzlich resultiert aus einer orofazialen Muskelhypotonie häufig ein offenstehender Mund mit heraushängender Zunge (Pseudomakroglossie), ein lebenslang persistierendes, viszerales Schluckmuster sowie eine prognathe Kieferlage. Der Hartgaumen ist oft sagittal verkürzt und stark gewölbt (Gothischer Gaumen) (Arumugam et al. 2016).

Viele der beschriebenen Gebisssituationen begünstigen die Akkumulation von Nahrungsresten und pathogenen Mikroorganismen. Die Durchführung von Mundhygienemaßnahmen ist bei den beschriebenen Zahn- und Kieferfehlstellungen erschwert. Personen mit kognitiver Einschränkung sind oft nicht oder nicht mehr in der Lage, selbstständig für die Aufrechterhaltung ihres Mundgesundheitszustandes durch entsprechende Mundhygienemaßnahmen zu sorgen. Diese Personen sind zur Kompensation ihres behinderungsassoziierten Pflegedefizits auf ihr betreuendes Umfeld angewiesen. Ein unkooperatives oder sogar aggressives Verhalten der Personen mit kognitiver Einschränkung kann die Durchführung von Mundhygienemaßnahmen zusätzlich erschweren. Gelingt die Kompensation des Pflegedefizits durch das soziale Umfeld nicht, sind das Auftreten von Karies und/oder Parodontitis die Folge. Die Mundgesundheit von Personen mit kognitiver Einschränkung ist folglich von behinderungsassoziierten somatischen Faktoren und dem Kompensationsverhalten der Betreuungspersonen (Ernährungslenkung, Zahnpflege) abhängig.

\subsection{Mundgesundheitszustand von Personen mit kognitiver Einschränkung}

Die Heterogenität des Personenkreises mit kognitiver Einschränkung erschwert die Einbeziehung großer einheitlicher Stichproben. Außerdem erschweren uneinheitliche Bezeichnungen der Personengruppen eine einheitliche Übersicht der Studienergebnisse. In epidemiologischen Studien wird die Personengruppe mit kognitiver Einschränkung als „Special Health Care Needs“ oder „Special Needs Patients“, „handicapped“, „intellectual disabled“, „developmental disabled“ oder „persons with mental illness“ bezeichnet. Zahnmedizinische Studien, 
die den Mundgesundheitszustand von erwachsenen Personen mit kognitiver Einschränkung bestimmen, beziehen sich meist auf kleine Untergruppen: Patienten, die eine Universitätsklinik aufsuchten (Gabre 2000); Personen, die an einer speziellen Sportveranstaltung für Menschen mit Behinderung teilnahmen (Bissar et al. 2010; Schulte et al. 2011; Fernandez et al. 2015; Marks et al. 2015); Personen, die in einer speziellen Einrichtung für Menschen mit Behinderung leben oder arbeiten (Pieper et al. 1986; Pezzementi und Fisher 2005; Leroy und Declerck 2013; Schulte et al. 2013; Hempel et al. 2015) oder Personen mit einer einheitlichen Diagnose, wie z. B. Autismus, Trisomie 21 oder Hirnschädigung als Folge eines Schlaganfalls (Cheng et al. 2007; Jaber 2011; Frydman und Nowzari 2012; Deps et al. 2015; Kothari et al. 2017). Je nach Personenklientel variieren die Ergebnisse zum Mundgesundheitszustand stark. Die systematische Übersichtsarbeit von Anders und Davis (2010) zeigt, dass dem zahnmedizinischen Behandlungsbedarf bei Personen mit kognitiver Einschränkung schlechter entsprochen wird als dem Behandlungsbedarf von Vergleichs- oder Kontrollgruppen ohne kognitive Einschränkung. In den berücksichtigten Studien war die Kariesprävalenz trotz der einheitlich schlechten Mundhygiene bei Personen mit kognitiver Einschränkung im Vergleich zu Vergleichs- oder Kontrollgruppen gleich oder sogar geringer. Zu erklären ist die verminderte Kariesrate bei gleichzeitig schlechter Mundhygiene durch gering kariogene und reglementierte Essgewohnheiten. Die vergleichsweise wenigen kariösen Läsionen waren allerdings häufiger unbehandelt als in den Vergleichsoder Kontrollgruppen. Außerdem wiesen Patienten mit kognitiver Einschränkung eine höhere Anzahl an fehlenden Zähnen und eine geringere Anzahl an Füllungen auf. Die hohe Anzahl an fehlenden Zähnen und die hohe Anzahl unversorgter kariöser Läsionen sprechen für einen schlechten Mundgesundheitszustand und einen nicht erfüllten zahnmedizinischen Behandlungsbedarf von Personen mit kognitiver Einschränkung. Kothari et al. (2017) zeigen ebenfalls in einer systematischen Übersichtarbeit, dass erwachsene Personen mit Hirnschädigung häufig eine reduzierte Mundgesundheit durch plaqueinduzierte Gingivitiden und eine hohe Anzahl fehlendender Zähne haben. Auch Personen mit schweren psychischen Erkrankungen haben aufgrund einer meist inadäquaten Mundhygiene häufig einen durch Karies und Parodontitis reduzierten Mundgesundheitszustand (Matevosyan 2010; Torales et al. 2017). Bei der Un- 
tersuchung von 707 erwachsenen Personen mit unterschiedlichen Behinderungen in Belgien stellen Leroy und Declerck (2013) einen unerfüllten präventiven und kurrativen zahnmedizinischen Behandlungsbedarf fest. Schulte et al. (2013) untersuchten den Mundgesundheitszustand von erwachsenen Personen mit kognitiver Einschränkung aus zwei Tageseinrichtungen in Deutschland und stellen eine im Vergleich zur Durchschnittsbevölkerung erhöhte Kariesprävalenz und eine wohnortabhängige zahnärztliche Versorgung fest. Personen mit kognitiver Einschränkung werden in Deutschland nicht in allen Regionen gleich gut zahnmedizinisch betreut. Generell zählen Personen mit Pflegebedarf - mit oder ohne kognitiver Einschränkung - auch in Deutschland zu der Personengruppe mit dem schlechtesten Mundgesundheitszustand und dem größten zahnmedizinischen Therapiebedarf (Jordan et al. 2016).

\subsection{Zahnärztliche Therapie von Personen mit kognitiver Einschränkung}

Kariöse Läsionen oder parodontale Erkrankungen bleiben bei Personen mit kognitiver Einschränkung häufig unbehandelt (Gabre 2000; Tiller et al. 2001; Anders und Davis 2010; Matevosyan 2010; Kothari et al. 2017). Laut der Befragung von Leroy und Declerck (2013) suchen Patienten mit Behinderung häufig keinen Zahnarzt auf, weil Ängste vor einer zahnmedizinischen Therapie, Ängste vor anfallenden Kosten oder Probleme bei dem selbstständigen Aufsuchen einer Zahnarztpraxis bestehen. Sind die Patienten durch ihre kognitive Einschränkung nicht in der Lage, (zahn-)medizinische Therapieentscheidungen selbstständig und reflektiert zu treffen, ist es in Deutschland die Aufgabe des gesetzlichen Vertreters für die Umsetzung einer adäquaten Gesundheitsfürsorge des Betreuten zu sorgen. Dies bedeutet, dass der gesetzliche Vertreter Zahnarzttermine koordiniert und gemeinsam mit dem behandelnden Zahnarzt Therapieentscheidungen im mutmaßlichen Interesse des Patienten (Bestinterests standard) trifft. Personen, die aufgrund einer kognitiven Einschränkung nicht selbstständig für ihren Gesunderhalt sorgen können, stellen eine besonders vulnerable Patientengruppe dar (Groß 2012). Je nach kognitivem Entwicklungsalter verhalten sich die Personen oft übermäßig ängstlich und reagieren 
abwehrend auf zahnärztliche Therapiemaßnahmen (Rud und Kisling 1973; Gordon et al. 1998). Wenn durch eine kognitive Einschränkung die Therapiefähigkeit, die Mundhygienefähigkeit und/oder die Eigenverantwortlichkeit des Patienten beeinträchtig sind, sollte die zahnärztliche Betreuung auf die aus der kognitiven Einschränkung resultierenden Bedürfnisse dieser Patienten (reduzierte zahnmedizinische funktionale Kapazität) abgestimmt werden (Nitschke und Hopfenmüller 1991; Nitschke und Kaschke 2011). Es wurden Behandlungsstrategien entwickelt, die dem primär unkooperativen Patienten die zahnärztliche Therapiesitzung erleichtern sollen. Hierzu zählen spezielle Formen der Verhaltens- und Gesprächsführung bis hin zur Hypnose. Zusätzlich kann durch eine leichte physikalische Unterstützung (Halten des Kopfes oder der Hände durch die Bezugsperson) dass oft reflektorische Abwehrverhalten reduziert und der Patient beruhigt werden. Wenn diese Therapieunterstützungen scheitern, stellt eine zusätzliche, medikamentöse Sedierung (orale Prämedikation, Lachgassedierung) eine weitere Option dar (Festa et al. 1993; Glassman 2009; Lyons 2009). Scheitern auch diese Maßnahmen, ist die zahnmedizinische Therapie im Rahmen einer ITN (Intubationsnarkose) die letzte, weil invasivste, Möglichkeit zur Behandlung oraler Beschwerden (Nunn et al. 1995; Pohl et al. 1996; Ananthanarayan et al. 1998; Dougherty 2009; Glassman et al. 2009; Wang et al. 2012; Mallineni und Yiu 2015; Lim und Borromeo 2017). Um zahnärztliche Behandlungen individuell planen und entsprechend der (reduzierten) zahnmedizinischen funktionellen Kapazität gestalten zu können, summieren Nitschke und Hopfenmüller (1991) die Einschränkungen der Patienten entsprechend ihren Belastungsstufen mit dem in Tabelle 2-1 dargestellten Konzept. 
Tabelle 2-1: Zahnmedizinische funktionelle Kapazität nach Nitschke und Hopfenmüller (2011). Bei Personen mit Belastungsstufe 1 oder 2 sind, mit einer an die Einschränkungen des Patienten angepassten Behandlungsstrategie, kurze Behandlungen möglich. Bei Patienten der Belastungsstufe 3 oder 4 ist die Kooperationsfähigkeit so stark reduziert, dass komplexe zahnmedizinische Therapien meist nur im Rahmen einer Narkose möglich sind.

\begin{tabular}{|c|l|l|l|l|}
\hline Belastungsstufe & $\begin{array}{l}\text { Therapie- } \\
\text { Fähigkeit }\end{array}$ & $\begin{array}{l}\text { Mundhygiene- } \\
\text { fähigkeit }\end{array}$ & $\begin{array}{l}\text { Eigenverant- } \\
\text { Wortlichkeit }\end{array}$ & $\begin{array}{l}\text { Behandlungs- } \\
\text { strategie }\end{array}$ \\
\hline $\mathbf{1}$ & normal & normal & normal & \\
\hline $\mathbf{2}$ & leicht reduziert & leicht reduziert & normal & \\
\hline 3 & stark reduziert & stark reduziert & reduziert & evtl. in Narkose \\
\hline 4 & nicht vorhanden & nicht vorhanden & keine & evtl. in Narkose \\
\hline
\end{tabular}

Eine umfassende zahnmedizinische Therapie in ITN beinhaltet eine professionelle Zahnreinigung, bei Bedarf ein subgingivales Debridement (Deep Scaling), Füllungstherapien und/oder Zahnextraktionen. Endodontische Behandlungen werden seltener durchgeführt (Mallineni und Yiu 2015). Um eine erneute Behandlung in ITN zu vermeiden, werden Zähne mit fraglicher Prognose (Verdacht auf Pulpitis oder Pulpanekrose, starke parodontale Schädigung) entfernt. Ziel der Therapie in Narkose ist die Schmerzbeseitigung sowie die langfristige Wiederherstellung der Mundgesundheit (orale Rehabilitation) bei maximalem Kaukomfort. Bisher wurden allgemeine Auswirkungen der zahnmedizinischen Therapie in ITN auf die Personen mit kognitiver Einschränkung wenig untersucht (Limeres Posse et al. 2003; Messieha et al. 2007; Mallineni und Yiu 2015; Chang und Kim 2017). 


\subsection{Mundgesundheitsbezogene Lebensqualität (MLQ)}

Die Bewertung und Wahrnehmung von Lebensqualität ist geprägt von sozialisationsbedingten Standards (extrinsischen Faktoren) und dem individuellen Charakter (intrinsischen Faktoren) (Locker 1988; Allen 2003, John et al. 2003, John et al. 2004, Abbildung 2-2).

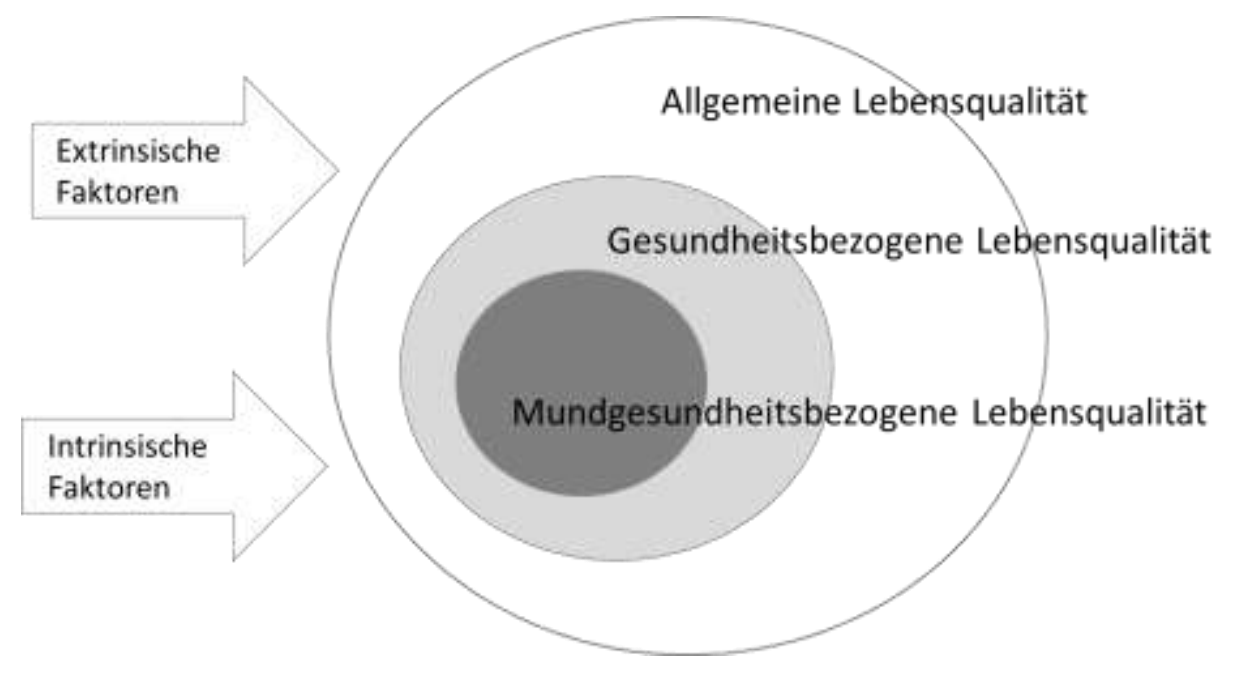

Abbildung 2-2: Mundgesundheitsbezogene Lebensqualität als Teilbereich der gesundheitsbezogenen und allgemeinen Lebensqualität nach John und Micheelis (2003). Sowohl intrinsische (personenbezogene) wie auch extrinsische (umweltbezogene) Faktoren beeinflussen die Ansprüche an die einzelnen Teilbereiche der Lebensqulität.

Die standardisierte Bestimmung der gesundheitsbezogenen Lebensqualität (Health-Related Quality of Life, HRQoL) ist ein patientenzentrierter Parameter zur Bestimmung des Patientenwohls im Verlauf medizinischer Therapien (Bullinger 1997; Bullinger 2002; Sousa und Kwok 2006). Die Instrumente zur Quantifizierung der gesundheitsbezogenen Lebensqualität kalibrieren sich auf die Definition der World Health Organization quality of life assessment (WHOQOL):

„Lebensqualität ist die subjektive Wahrnehmung einer Person über ihre Stellung im Leben in Relation zur Kultur und den Wertesystemen in denen sie lebt und in Bezug auf ihre Ziele, Erwartungen, Standards und Anliegen." (WHO 1995) 
Die gesundheitsbezogene Lebensqualität gilt als ein multidimensionales Konstrukt aus physischen, psychischen, mentalen, sozialen, funktionalen Dimensionen sowie einer Einschätzung über die Wahrnehmung des persönlichen Gesundheitszustandes durch den Patienten selbst (Bullinger 2002). Bei Patienten mit chronischen Allgemeinerkrankungen konnte gezeigt werden, dass ein Rückgang klinischer Symptome nicht automatisch ein verbessertes Wohlbefinden für den Patienten bedeutet (Osoba et al. 1994). Bei einer Befragung von erwachsenen Personen mit Hirnschädigung zeigte sich, dass die gesundheitsbezogene Lebensqualität positiv wahrgenommen wird, wenn durch die mit der Hirnschädigung einhergehenden reduzierten gesundheitlichen und kognitiven Fähigkeiten akzeptiert werden (Van Bost et al. 2017). Neben klinischen Symptomen bestimmen vor allem psychosoziale und kulturelle Einflussfaktoren die Wahrnehmung und Bewertung der eigenen Gesundheit (Wilson und Cleary 1995). Im Bereich der Zahnmedizin ist die Wahrnehmung von Mundgesundheit und die Bewertung des Mundgesundheitszustandes von Interesse (John 2007). Hierzu hat sich die mundgesundheitsbezogene Lebensqualität (MLQ, Oral Health-Related Quality of Life, OHRQoL) als psychosozialer Parameter im wissenschaftlichen Kontext etabliert (Strassburger et al. 2004; Naito et al. 2006). Locker (1988) beschreibt, mit dem in Abbildung 2-3 dargestellten Modell, die Ursachen für eine Reduktion der MLQ. Orale Erkrankungen können zu Schmerzen, Funktionseinschränkungen beim Essen und Sprechen, Scham in Bezug auf die dentale Ästhetik sowie zu einem reduzierten Selbstbewusstsein und damit zu einer reduzierten MLQ führen. Locker (1988) berücksichtigt keine psychosozialen und kulturellen Einflussfaktoren und leitet eine Reduktion der MLQ als eine hierarchische Folge oraler Erkrankung ab. Er geht nicht darauf ein, woraus sich die persönlichen Anforderungen an die MLQ zusammensetzten. 


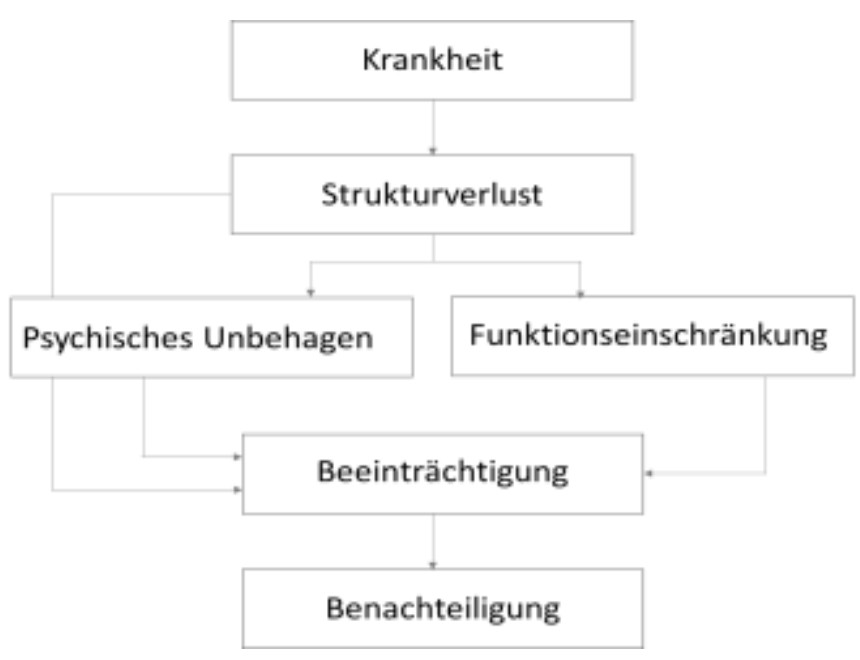

Abbildung 2-3: Theoretisches Modell zur MLQ nach Locker (1988). In einer hierarchischen Abfolge werden die Ursachen für eine reduzierte mundgesundheitsbezogene Lebensqualität aufgezählt.

Mittlerweile wurden komplexere theoretische Modelle mit einer unterschiedlichen Anzahl an Dimensionen zur Erklärung der MLQ entwickelt. In Anlehnung an das Verständinis zur gesundheitsbezogenen Lebensqualität beschreiben Sischo und Broder (2011) die individuelle Bewertung der MLQ im Kontext weiterer Einflussfaktoren (Abbildung 2-4). Sie nennen neben intrinsischen und extrinsischen Faktoren auch die Ätiologie einer eventuell vorhandenen Grunderkrankung als potenziellen Einflussfaktor auf die individuellen Ansprüche an die MLQ. 


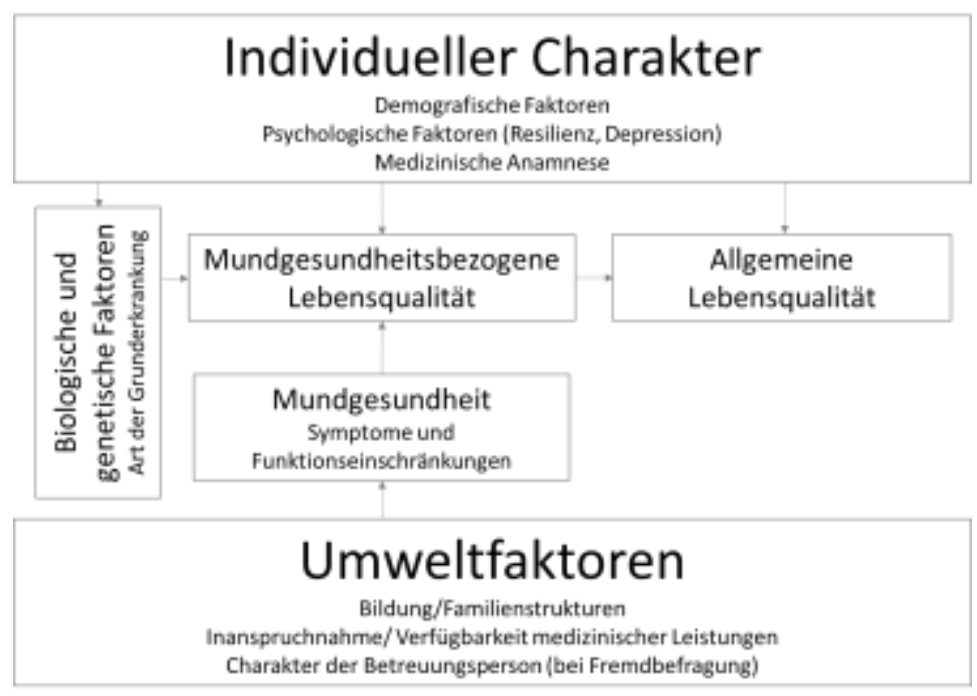

Abbildung 2-4: Theoretisches Modell nach Sischo und Border (2011) zur Beschreibung von mundgesundheitsbezogener und allgemeiner Lebensqualität als Resultat verschiedener Einflussfaktoren.

Das individuelle Niveau der Grundbedürfnisse der MLQ wird davon beeinflusst, wie wichtig dem Einzelnen die Gesundheit des orofazialen Systems ist und wie stark er Einschränkungen im Alltag durch den Mundgesundheitszustand empfindet bzw. wie stark seine Fähigkeiten zur Problembewältigung (Coping) ausgebildet sind (Allison et al. 1997). Davis et al. (2000) untersuchten den Einfluss psychosozialer Faktoren, indem sie Patienten mit identischen Befunden zu ihrer MLQ befragten. Alle Patienten waren komplett zahnlos, aber nur $42 \%$ beschrieben in diesem Zusammenhang negative Auswirkungen auf ihr Selbstbewusstsein und ihren Lebensalltag. Personen sind in ihrer MLQ benachteiligt, wenn sie beispielsweise aus Scham über Zahnlücken soziale Kontakte vermeiden oder aufgrund einer Siallorrhö (,feuchte Aussprache“ oder "Sabbern“) von ihrem sozialen Umfeld gemieden werden. Die Anspruchshaltung an die eigene Mundgesundheit wird von der individuellen sozialen und kulturellen Prägung modelliert (Cushing et al. 1986).

Steele et al. (2004) stellten fest, dass australische Patienten bereits bei vier fehlenden Zähnen Einschränkungen der MLQ aufwiesen, während britische Patienten erst bei elf fehlenden Zähnen in ihrer MLQ eingeschränkt waren. Es ist von einer sozialisationsspezifischen Bewertung der MLQ auszugehen. 
Idealerweise verbessert sich durch eine zahnmedizinische Therapie das subjektive Wohlbefinden und die mundgesundheitsbezogene Lebensqualität des Patienten. Nicht nur die medizinisch optimale Versorgung, sondern auch die Erwartungshaltung des Patienten an die zahnmedizinische Therapie hat einen wesentlichen Einfluss auf den gewünschten Zugewinn an MLQ. Allen et al. (2001) belegen, dass zahnlose Patienten, die sich eine Implantatversorgung wünschten, aber mit konventionellen Prothesen therapiert wurden, einen geringeren Zugewinn an MLQ hatten, als Patienten, die initial den Wunsch nach konventionellen Prothesen äußerten und diese Prothesen auch erhielten. Der objektiv beurteilbare klinische Befund und die subjektive Wahrnehmung der Patienten differieren häufig. Die Bewertung der MLQ generell sowie im Verlauf einer zahnmedizinischen Therapie ist ein dynamischer Prozess und wird von diversen patientenabhängigen Einflussfaktoren modelliert (Allen et al. 2001). Ursprünglich wurde der Erfolg einer zahnmedizinischen Therapie und die zahnmedizinische Versorgungssituation von Patienten- und Personengruppen ausschließlich über klinische Befunde erfassende Indizes beschrieben. Das Konzept der MLQ ermöglicht es, analog zu dem Konzept der gesundheitsbezogenen Lebensqualität, therapeutische Interventionen zur Verbesserung von Gesundheit und Lebensqualität auf Individuums- sowie Bevölkerungsebene zu evaluieren und gibt einen Überblick über die Versorgungssituation von Personen- oder Patientengruppen (Bullinger 1997; Corson et al. 1999; Bullinger 2000; Bullinger 2002; John et al. 2003; Locker et al. 2004; Brauchle et al. 2013; Jenei et al. 2015; Locker und Gibson 2006).

\subsection{Oral Health Impact Profile (OHIP)}

Instrumente der gesundheitsbezogenen Lebensqualität erfassen die vielschichtigen Dimensionen der MLQ nicht adäquat (Allen et al. 1999). Deshalb wurden spezielle Instrumente zur Bestimmung der MLQ entwickelt. Diese Fragebogeninstrumente unterscheiden sich in ihrer Anzahl an Fragen und in ihren Einsatzgebieten. Einige Messinstrumente quantifizieren einzelne Dimensionen der MLQ, beispielsweise Ästhetik oder soziale Folgen im Zusammenhang mit der MLQ. Andere Messinstrumente sind auf die Bedürfnisse und Ansprüche spezi- 
eller Personengruppen (Senioren, Kinder, verschiedene Nationalitäten) abgestimmt (Müller und Nitschke 2010).

Auf Basis des Modells von Locker (1988) wurde das Oral Health Impact Profile (OHIP) von Slade und Spencer (1994) konzipiert. Der OHIP-Fragebogen dient der Dokumentation von sozialen und funktionellen Einschränkungen im Zusammenhang mit der Mundgesundheit von Einzelpersonen und Patientengruppen sowie der Evaluation zahnärztlicher Therapiemaßnahmen. Der OHIP hat sich in verschiedenen Modifikationen und Versionen international als Fragebogeninstrument zur Quantifizierung der MLQ etabliert (Slade und Spencer 1994; Slade et al. 1996; Awad et al. 2000; John et al. 2002; Slade 2002; John et al. 2003; Hassel et al. 2008; Sierwald et al. 2011; Bettie et al. 2015; Malter et al. 2015). Die OHIP-Fragebögen quantifizieren die MLQ generisch und erfassen funktionelle Aspekte, Schmerzen, Einschränkungen der Ästhetik und des sozialen Lebens sowie psychologische Auffälligkeiten im Zusammenhang mit der Mundgesundheit. Die englische Originalversion (OHIP-E49) umfasst 49 Items. John et al. (2003) übersetzten den englischen Fragebogen in die deutsche Sprache. Um auch kulturelle Faktoren in der Übersetzung zu berücksichtigen, wurden vier Items ergänzt. Der deutsche Originalfragebogen (OHIP-G53) besteht deshalb aus 53 Items (John et al. 2003). Zur Integration der Befragungen in den klinischen Alltag wurden gekürzte Versionen entwickelt. Eine deutsche Version mit 14 Items entspricht der direkten Übersetzung der englischen Kurzversion (Slade 1997). Der OHIP-14 misst trotz der reduzierten Anzahl an Items ähnlich genau wie die Langversion (Awad et al. 2000; Allen 2003; John et al. 2003). In Tabelle 2-2 ist die deutsche Version mit 14 Fragen (OHIP-G14) dargestellt. 
Tabelle 2-2: Oral-Health-Impact-Profile-German-14 (OHIP-G14). Fragebogen zur Bestimmung der MLQ, deutsche Version mit 14 Items zur Bestimmung der MLQ. Die Auswertung erfolgt durch die Addition der einzelnen Itempunkte (0-4) zu einem Summenwert (0-56).

\begin{tabular}{|c|c|c|c|c|c|}
\hline $\begin{array}{l}\text { Hatten Sie im vergangenen Monat aufgrund von Problemen mit } \\
\text { Ihren Zähnen, im Mundbereich oder mit Ihrem Zahnersatz... }\end{array}$ & $\begin{array}{l}\text { sehr } \\
\text { oft }\end{array}$ & oft & $\begin{array}{l}\text { ab } \\
\text { und } \\
\text { zu }\end{array}$ & kaum & nie \\
\hline $\begin{array}{l}\text { Schwierigkeiten bestimmte Worte } \\
\text { auszusprechen? }\end{array}$ & 4 & 3 & 2 & 1 & 0 \\
\hline $\begin{array}{l}\text { das Gefühl, Ihr Geschmackssinn war } \\
\text { beeinträchtigt }\end{array}$ & 4 & 3 & 2 & 1 & 0 \\
\hline $\begin{array}{l}\text { den Eindruck, dass Ihr Leben ganz allgemein weniger zufriedenstel- } \\
\text { lend war }\end{array}$ & 4 & 3 & 2 & 1 & 0 \\
\hline Schwierigkeiten zu entspannen? & 4 & 3 & 2 & 1 & 0 \\
\hline $\begin{array}{l}\text { Ist es im vergangenen Monat aufgrund von Problemen mit Ihren } \\
\text { Zähnen, im Mundbereich oder mit Ihrem Zahnersatz vorgekom- } \\
\text { men, ... }\end{array}$ & $\begin{array}{l}\text { sehr } \\
\text { oft }\end{array}$ & oft & $\begin{array}{l}\text { ab } \\
\text { und } \\
\text { zu }\end{array}$ & kaum & nie \\
\hline dass Sie sich angespannt gefühlt haben? & 4 & 3 & 2 & 1 & 0 \\
\hline $\begin{array}{l}\text { dass Sie Ihre Mahlzeiten unterbrechen } \\
\text { mussten? }\end{array}$ & 4 & 3 & 2 & 1 & 0 \\
\hline $\begin{array}{l}\text { dass es Ihnen unangenehm war, bestimmte Nahrungsmittel zu } \\
\text { essen }\end{array}$ & 4 & 3 & 2 & 1 & 0 \\
\hline dass Sie anderen Menschen gegenüber eher reizbar gewesen sind? & 4 & 3 & 2 & 1 & 0 \\
\hline $\begin{array}{l}\text { dass es Ihnen schwergefallen ist, Ihren } \\
\text { alltäglichen Beschäftigungen nachzugehen? }\end{array}$ & 4 & 3 & 2 & 1 & 0 \\
\hline dass Sie vollkommen unfähig waren, etwas zu tun? & 4 & 3 & 2 & 1 & 0 \\
\hline $\begin{array}{l}\text { dass Sie sich ein wenig verlegen gefühlt } \\
\text { haben? }\end{array}$ & 4 & 3 & 2 & 1 & 0 \\
\hline dass Ihre Ernährung unbefriedigend gewesen ist? & 4 & 3 & 2 & 1 & 0 \\
\hline Hatten Sie im vergangenen Monat... & $\begin{array}{l}\text { sehr } \\
\text { oft }\end{array}$ & oft & $\begin{array}{l}\text { ab } \\
\text { und } \\
\text { zu }\end{array}$ & kaum & nie \\
\hline Schmerzen im Mundbereich? & 4 & 3 & 2 & 1 & 0 \\
\hline $\begin{array}{l}\text { ein Gefühl der Unsicherheit in Zusammenhang mit Ihren Zähnen, } \\
\text { Ihrem Mund oder Ihrem Zahnersatz }\end{array}$ & 4 & 3 & 2 & 1 & 0 \\
\hline
\end{tabular}


Der in Tabelle 2-3 dargestellte OHIP-G5 ist die auf fünf Items gekürzte maximale Kurzversion des OHIPs. Der OHIP-G5 erfasst trotz der stark reduzierten Anzahl an Antwortmöglichkeiten noch 90 \% der im Summenwert der Langversion enthaltenen Informationen und ist damit als Instrument zur Quantifikation der MLQ geeignet. Der OHIP-G5 wird aufgrund des geringen Zeitaufwandes besonders zur Integration der Befragung in den klinischen Alltag empfohlen (John 2004; Sierwald et al. 2011; John et al. 2014; Waller et al. 2016).

Tabelle 2-3: Oral Health-Impact-Profile-German-5 (OHIP-G5). Deutsche Version des OHIP mit fünf Fragen. Die Auswertung erfolgt durch die Addition der einzelnen Itempunkte (0-4) zu einem Summenwert (0-20).

\begin{tabular}{|c|c|c|c|c|c|}
\hline $\begin{array}{l}\text { Hatten Sie aufgrund von Problemen mit Ihren Zähnen, im } \\
\text { Mundbereich, Kiefer oder mit Ihrem Zahnersatz... }\end{array}$ & $\begin{array}{l}\text { sehr } \\
\text { oft }\end{array}$ & oft & $\begin{array}{l}\text { ab } \\
\text { und } \\
\text { zu }\end{array}$ & kaum & nie \\
\hline Schwierigkeiten beim Kauen bestimmter Lebensmittel? & 4 & 3 & 2 & 1 & 0 \\
\hline den Eindruck, Ihr Essen war geschmacklich weniger gut? & 4 & 3 & 2 & 1 & 0 \\
\hline $\begin{array}{l}\text { den Eindruck, dass Ihr Leben ganz allgemein weniger zufriedenstel- } \\
\text { lend war? }\end{array}$ & 4 & 3 & 2 & 1 & 0 \\
\hline Hatten Sie in den letzten 7 Tagen... & $\begin{array}{c}\text { sehr } \\
\text { oft }\end{array}$ & oft & $\begin{array}{c}\text { ab } \\
\text { und } \\
\text { zu }\end{array}$ & kaum & nie \\
\hline Schmerzen im Mundbereich? & 4 & 3 & 2 & 1 & 0 \\
\hline $\begin{array}{l}\text { wegen des Aussehens Ihrer Zähne oder Ihres Zahnersatzes sich } \\
\text { unwohl/unbehaglich gefühlt? }\end{array}$ & 4 & 3 & 2 & 1 & 0 \\
\hline $\begin{array}{l}\text { Ist es Ihnen in den letzten } 7 \text { Tagen aufgrund von Problemen mit } \\
\text { Ihren Zähnen, im Mundbereich, Kiefer oder mit Ihrem Zahner- } \\
\text { satz... }\end{array}$ & $\begin{array}{c}\text { sehr } \\
\text { oft }\end{array}$ & oft & $\begin{array}{l}\text { ab } \\
\text { und } \\
\text { zu }\end{array}$ & kaum & nie \\
\hline $\begin{array}{l}\text { schwergefallen, Ihren alltäglichen Beschäftigungen } \\
\text { nachzugehen? }\end{array}$ & 4 & 3 & 2 & 1 & 0 \\
\hline
\end{tabular}

Alle OHIP-Versionen messen die Reduktion der MLQ über die vom Patienten angegebenen Einschränkungen. Die einzelnen Items erfragen Folgen oraler Erkrankungen. Jedes Item soll vom Patienten entsprechend einer Fünfstufenskala beantwortet werden. Die Einstufung „nie“ entspricht dem Zahlenwert „null“ und die Einstufung „sehr oft“ entspricht dem Zahlenwert „vier“. Zur Auswertung des Fragebogens wird ein Summenwert (Score) gebildet. Der Summenwert spiegelt die Einschränkungen, die im Zusammenhang mit der Mundgesundheit empfunden werden, wider und erfasst so quantitativ die Reduktion der MLQ. Dies ermöglicht Interpretationen und Vergleiche von Auswirkungen einer veränderten Mundgesundheit auf die Lebensqualität von Patienten und Patientengruppen (Locker 1988; Locker und Slade 1994; Awad et al. 2000; Thomson et 
al. 2012; John et al. 2016). Der Maximalwert des jeweiligen Fragebogens (OHIP-G14: 56 Punkte; OHIP-G5: 20 Punkte) entspricht einer maximal eingeschränkten MLQ. Ein Score von null bedeutet, dass keinerlei Einschränkungen der MLQ angegeben wurden. Patienten mit identischem Summenwert haben eine identisch reduzierte $M L Q$, allerdings kann die Ursache für die reduzierte MLQ unterschiedlich sein. John et al. (2004) validierten den OHIP-G14 und den OHIP-G5 durch eine Stichprobenbefragung von 2050 Bundesbürgern im Alter von 16 bis 79 Jahren und stellten außerdem für beide Versionen Durchschnittswerte der deutschen Allgemeinbevölkerung vor. Neunzig Prozent der Befragten hatten einen OHIP-G5-Score von $\leq 4$ und einen OHIP-G14-Score $\leq$ 11. Personen mit einer reduzierten Zahnanzahl und Zahnersatz hatten generell höhere Scores, waren in ihrer MLQ also eingeschränkter als Personen mit eigenen Zähnen und ohne Zahnersatz. Eine Gewichtung der einzelnen Fragen wird für die deutsche Version nicht empfohlen. Der OHIP wurde bei unterschiedlichen Personengruppen, wie Senioren, Angstpatienten, Schwangeren, sowie zur Evaluation verschiedener zahnmedizinischer Therapieverfahren eingesetzt (John et al. 2003; Jensen et al. 2008; Schmidt et al. 2013; 2014; Fischer et al. 2015; Jang et al. 2015; Kolenda et al. 2016; Wöstmann et al. 2016; Mühlberg et al. 2017; Schmalz et al. 2017; Schweyen et al. 2017; Stelzle et al. 2017).

\subsection{Mundgesundheitsbezogene Lebensqualität von Patienten mit kognitiver Einschränkung}

Wie in den vorherigen Abschnitten beschrieben, sind Personen mit kognitiver Einschränkung in Bezug auf Einbußen ihrer Gesundheit und Mundgesundheit besonders gefährdet. Behinderungsassoziierte Faktoren beeinflussen die (körperlichen) Lebensbedingungen und vermutlich auch die Wahrnehmung und Bewertung dieser (Kane et al. 2003; Hassel et al. 2007; Jensen et al. 2008; Jang et al. 2015). Es ist unklar, welche behinderungsassoziierten Faktoren die Bewertung der MLQ von Personen mit kognitiver Einschränkung beeinflussen. Spezielle Fragebögen zur Erfassung der MLQ von Patienten mit kognitiver Einschränkung existierten zu Beginn der vorliegenden Studie nicht. Ist der Patient so stark eingeschränkt, dass er selbst keine Aussagen zu seiner MLQ machen 
kann, bleibt zur Quantifikation der MLQ nur die Fremdbefragung über eine Betreuungsperson. Eine Fremdbefragung beinhaltet allerdings immer auch Risiken der Fehleinschätzung, da eventuelle Wertvorstellungen und Charktereigenschaften der Betreungsperson die Bewertung der MLQ beeinflussen (Eiser und Morse 2001; Barbosa und Gavião 2008; Sischo und Broder 2011; Lee et al. 2017). Hauptsächlich wird die Fremdbefragung zur Quantifikation der MLQ von Kindern eingesetzt. In einer systematischen Literaturübersicht stellen Barbosa und Gavião (2008) fest, dass Eltern besonders das psychosoziale und emotionale Wohlbefinden im Zusammenhang mit der MLQ anders als ihre Kinder bewerten. Eltern von Kindern mit geistiger Behinderung entwickeln verschiedene Copingstrategien im Umgang mit der Behinderung ihres Kindes (Doege 2017). Studien deuten darauf hin, dass das Copingverhalten der Eltern das Risiko für Verzerrungen der Fremdbewertung der MLQ erhöht. Dies stellen El Ashiry et al. (2016) bei einer Befragung von Eltern von Kindern mit infantiler Cerebralparese fest. Obwohl die Mundgesundheit vergleichbar zu der Mundgesundheit einer regulär entwickelten Vergleichsgruppe war, bewerteten die Eltern der Kinder mit infantiler Cerebralparese die MLQ ihrer Kinder schlechter als die Eltern der gesunden Kinder. Eltern von Kindern mit Gesichtsspalten schätzen die MLQ ihrer Kinder schlechter ein als die Kinder selbst (Kramer et al. 2008).

Über Fremdbefragung konnte allerdings festgestellt werden, dass ein zahnmedizinischer Behandlungsbedarf die MLQ von Personen mit kognitiver Einschränkung negativ beeinflusst (Loureiro et al. 2007; Alves Nayara Silva et al. 2016). Bislang befragte nur eine koreanische Studie Betreuungspersonen von Personen mit kognitiver Einschränkung im Hinblick auf die Auswirkungen der zahnmedizinischen Behandlung in Intubationsnarkose (ITN). Es konnte ein positiver Einfluss der zahnmedizinischen Therapie auf die Bewertung der MLQ durch die Betreuungspersonen festgestellt werden (Chang et al. 2014).

Die empirische Sozialforschung im Kontext geistiger Behinderung bewertet die Erforschung von Fragestellungen in Bezug auf die Lebensqualität von Personen mit kognitiver Einschränkung allein durch die Fremdeinschätzung von Eltern oder Betreuern kritisch und fordert ein personenzentriertes Einbeziehen dieser speziellen Personengruppe in Forschungsarbeiten (Janz und Terfloth 2009). Couto et al. (2018) befragten Personen mit leichter kognitiver Einschränkung selbst zu ihrer MLQ und stellen fest, dass diese Personen ihre MLQ plau- 
sibel bewerten können. Die befragten Personen bewerteten ihre MLQ schlechter, wenn ein zahnmedizinischer Behandlungsbedarf bestand. Es wurde bisher nicht versucht, Personen, die aufgrund ihrer kognitiven Einschränkung im Rahmen einer ITN zahnmedizinisch behandelt werden müssen, selbst zu ihrer MLQ zu befragen. Es ist unbekannt, ob Personen, die aufgrund ihrer kognitiven Einschränkung nur im Rahmen einer ITN zahnmedizinisch behandelt werden können, Aussagen zu ihrer MLQ machen können und, wenn ja, ob diese Aussagen mit den Fremdbewertungen der Betreuungspersonen korrelieren. Es ist ebenfalls unklar, wie sich die komplexe zahnmedizinische Therapie im Rahmen einer ITN auf die Bewertung der MLQ auswirkt.

\subsection{Ziel der Studie}

Die vorliegende Studie hatte zum Ziel, den Einfluss der zahnmedizinischen Behandlung im Rahmen einer Intubationsnarkose auf die mundgesundheitsbezogene Lebensqualität von Personen mit kognitiver Einschränkung zu untersuchen. Dafür sollten sowohl die Patienten selbst (sofern möglich) als auch deren Betreuer vor und nach der zahnmedizinischen Rehabilitation befragt werden. Außerdem sollten potenzielle Korrelationen hinsichtlich der Bewertung durch Patienten und Betreuern untersucht werden. Ferner sollte analysiert werden, welche Faktoren die Bewertung der MLQ bei dieser speziellen Patientenklientel beeinflussen. 


\section{Material und Methoden}

\subsection{Studienplan}

Bei der vorliegenden Untersuchung handelte es sich um eine prospektive Fragebogenstudie zur Bestimmung der mundgesundheitsbezogenen Lebensqualität von Patienten mit kognitiver Einschränkung vor und nach zahnmedizinischer Therapie in Intubationsnarkose (ITN). Das Forschungsvorhaben wurde von der Ethikkommission der Universitätsmedizin Göttingen genehmigt (Nummer 11/1/16).

Zur Teilnahme an der Studie wurden erwachsene Patienten mit kognitiver Einschränkung und deren rechtliche Betreuungspersonen angefragt, die sich im Zeitraum von März 2016 bis Januar 2018 in der Poliklinik für Präventive Zahnmedizin, Parodontologie und Kariologie der Universitätsklinik Göttingen vorstellten, und bei denen eine zahnmedizinische Behandlung in ITN geplant war.

Eine zahnmedizinische Behandlung im Wachzustand war aufgrund des geringen Kooperationsverhaltens der Patienten nicht möglich. Die in der Studie berücksichtigten Patienten entsprachen durch ihre kognitive Einschränkung der Belastungsstufe 4 nach Nitschke und Hopfenmüller (1991). 
Folgende Einschlusskriterien zur Studienteilnahme wurden definiert:

- Volljährige Patienten ( $\geq 18$ Jahre), die aufgrund einer kognitiven Einschränkung einen vom Betreuungsgericht festgelegten rechtlichen Betreuer in dem Bereich "Gesundheitsfürsorge" haben.

- Bestehende Indikation zur zahnärztlichen Behandlung in ITN aufgrund mangelnder Therapiekooperation.

- Schriftliche Einwilligung des gesetzlichen Betreuers zur Therapie in ITN sowie zur Studienteilnahme.

Folgende Ausschlusskriterien wurden definiert:

- Konventionelle zahnmedizinische Behandlung möglich (keine ITN).

- Minderjährige Patienten (<18 Jahre).

- Patienten ohne kognitive Einschränkung, ohne gesetzliche Betreuungsperson.

- Fehlendes Einverständnis zur Studienteilnahme.

Vor der Teilnahme an der Studie wurden die Patienten und deren gesetzliche Betreuer in einem Aufklärungsgespräch über Wesen und Tragweite der Studie informiert. Alle gesetzlichen Betreuer wurden zusätzlich schriftlich aufgeklärt und gaben ihr schriftliches Einverständnis.

Die Befragungen der Patienten und der Betreuer fand im Rahmen der Spezialsprechstunde für Menschen mit Behinderung der Poliklinik für Präventive Zahnmedizin, Parodontologie und Kariologie der Universitätsmedizin Göttingen statt. Die MLQ wurde vor der Therapie in ITN bei der Erstvorstellung zur Diagnostik und Therapieplanung des operativen Eingriffs (T0, Baseline) bestimmt. Die erneuten Befragungen erfolgten im Rahmen der regulären Nachkontrollen innerhalb von 2-3 (T1) und 12 Wochen (T2) postoperativ (Abbildung 3-1). Die Patienten wurden mit einem Fragebogen in leichter Sprache (OHIP-Ge) selbst befragt. Die jeweiligen Betreuer wurden mit dem OHIP-G14 (Fremdbefragung) befragt. Um eine gegenseitige Beeinflussung während der Befragung zu vermeiden, wurden die Betreuer räumlich getrennt von den Patienten befragt. Kli- 
nische Parameter zum Mundgesundheitszustand sowie Informationen über die in ITN erfolgten Therapiemaßnahmen sowie demographische und behinderungsassoziierte Daten wurden der Aktendokumentation (Anamnesebogen, Befundbogen und Akteneintrag) entnommen.
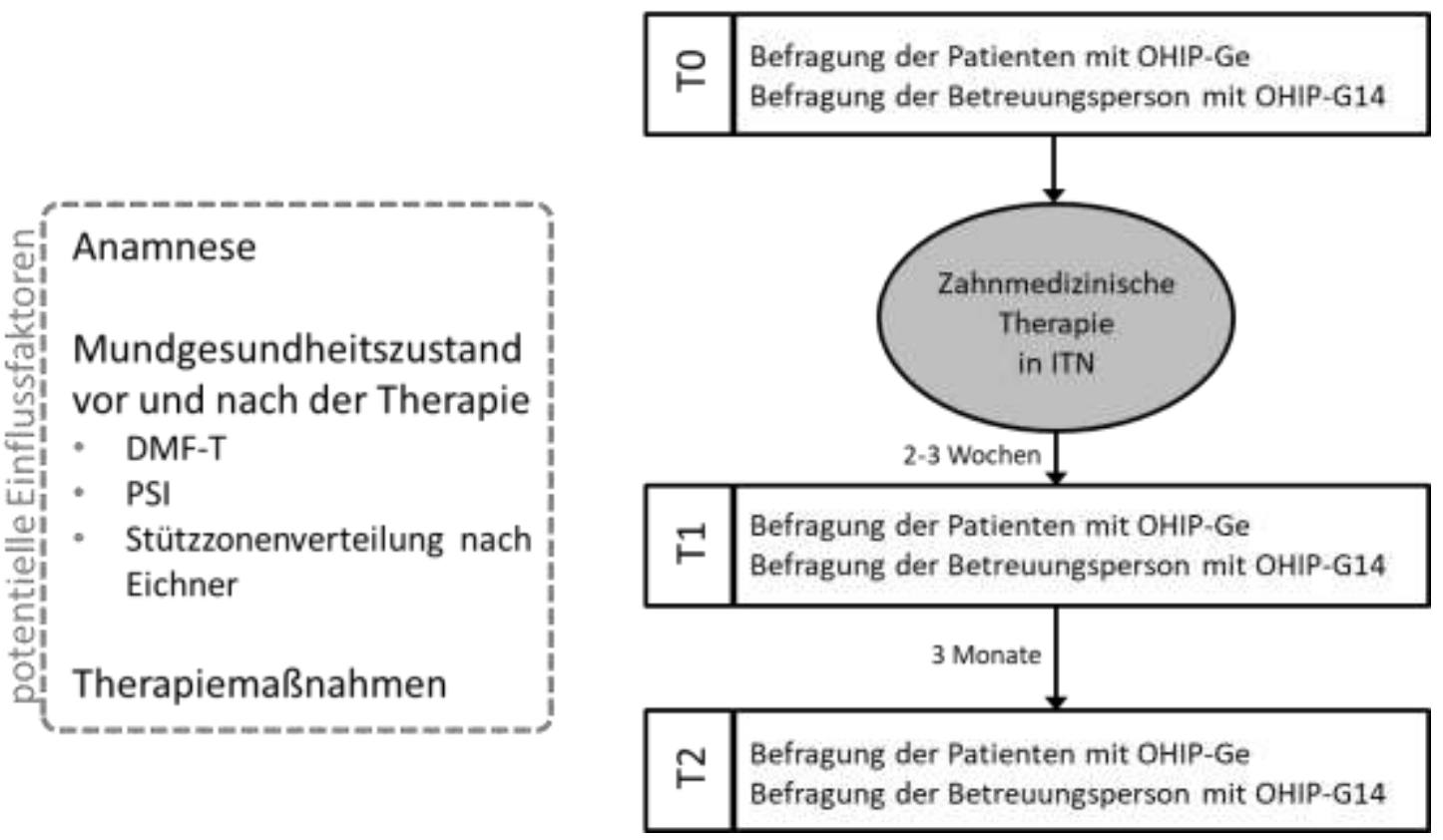

Abbildung 3-1: Studienplan. Befragung zur MLQ vor und nach der regulären zahnmedizinischen Therapie sowie Erfassung potenzieller Einflussfaktoren auf die MLQ aus der Aktendokumentation. 


\subsection{Bestimmung der MLQ durch Fremdbefragung}

Als Betreuer wurde jene Person befragt, die den Patienten im Alltag begleitet. Die Anzahl der Betreuungsstunden pro Tag wurde auf dem Betreuerfragebogen vermerkt. Für die schriftliche Befragung des Betreuers wurde eine an die Fremdbefragung angepasste Version des OHIP-G14 eingesetzt (John et al. 2002; John et al. 2003). Hilfestellungen bei Nichtverständnis einzelner Fragen waren jederzeit möglich. Der Betreuer wurde instruiert, sich bei der Beantwortung der Fragen zum Zeitpunkt T0 und T2 jeweils auf den Zeitraum der letzten vier Wochen zu beziehen. Bei der Befragung T1 wurde der Betreuer gebeten, auf den Zeitraum nach der Therapie Bezug zu nehmen. Die Häufigkeiten bestehender Einschränkungen konnten entsprechend den für den OHIP üblichen fünf Stufen („nie“, „kaum“, „ab und zu“, „oft“, „sehr oft“) angegeben werden. Zur Auswertung wurde jeder Antwortmöglichkeit ein Wert (0, 1, 2, 3 oder 4) zugeordnet (Fragenwert). Die einzelnen Werte wurden zu dem OHIP-G14Summenwert (Minimum: 0 = keine Einschränkung der MLQ; Maximum: 56 = maximale Einschränkung der MLQ) addiert (Tabelle 3-1). 
Tabelle 3-1: Angepasster OHIP-G14-Fragebogen zur Fremdbefragung der Betreuungspersonen. Die Itempunkte werden zur Auswertung zu einem Summenwert addiert. Der Maximalwert von 56 entspricht einer maximal eingeschränkten MLQ.

\begin{tabular}{|c|c|c|c|c|c|}
\hline $\begin{array}{l}\text { Hatten Sie den Eindruck, dass die von Ihnen betreute Person } \\
\text { aufgrund von Problemen mit Ihren Zähnen, im Mundbereich } \\
\text { oder mit Ihrem Zahnersatz... }\end{array}$ & $\begin{array}{l}\text { sehr } \\
\text { oft }\end{array}$ & oft & $\begin{array}{l}\text { ab } \\
\text { und } \\
\text { zu }\end{array}$ & kaum & nie \\
\hline Schwierigkeiten hatte, bestimmte Worte auszusprechen? & 4 & 3 & 2 & 1 & 0 \\
\hline einen beeinträchtigten Geschmackssinn hatte? & 4 & 3 & 2 & 1 & 0 \\
\hline allgemein weniger zufrieden war? & 4 & 3 & 2 & 1 & 0 \\
\hline Schwierigkeiten hatte zu entspannen? & 4 & 3 & 2 & 1 & 0 \\
\hline $\begin{array}{l}\text { Ist es aufgrund von Problemen mit Ihren Zähnen, im Mundbe- } \\
\text { reich oder mit Ihrem Zahnersatz vorgekommen, dass die von } \\
\text { Ihnen betreute Person ... }\end{array}$ & $\begin{array}{l}\text { sehr } \\
\text { oft }\end{array}$ & oft & $\begin{array}{l}\text { ab } \\
\text { und } \\
\text { zu }\end{array}$ & kaum & nie \\
\hline sich angespannt gefühlt hat? & 4 & 3 & 2 & 1 & 0 \\
\hline Mahlzeiten unterbrechen musste? & 4 & 3 & 2 & 1 & 0 \\
\hline Schwierigkeiten hatte, bestimmte Nahrungsmittel zu essen? & 4 & 3 & 2 & 1 & 0 \\
\hline anderen Menschen gegenüber eher reizbar gewesen ist? & 4 & 3 & 2 & 1 & 0 \\
\hline alltägliche Beschäftigungen schlechter ausüben konnte? & 4 & 3 & 2 & 1 & 0 \\
\hline vollkommen unfähig war, etwas zu tun? & 4 & 3 & 2 & 1 & 0 \\
\hline sich verlegen gefühlt hat? & 4 & 3 & 2 & 1 & 0 \\
\hline die Ernährung unbefriedigend gewesen ist? & 4 & 3 & 2 & 1 & 0 \\
\hline Bestanden bei der von Ihnen betreuten Person... & $\begin{array}{l}\text { sehr } \\
\text { oft }\end{array}$ & oft & $\begin{array}{l}\text { ab } \\
\text { und } \\
\text { zu }\end{array}$ & kaum & nie \\
\hline Schmerzen im Mundbereich? & 4 & 3 & 2 & 1 & 0 \\
\hline $\begin{array}{l}\text { Unsicherheiten in Zusammenhang mit den Zähnen, ihrem Mund } \\
\text { oder ihrem Zahnersatz? }\end{array}$ & 4 & 3 & 2 & 1 & 0 \\
\hline
\end{tabular}




\subsection{Bestimmung der MLQ durch Selbstbefragung}

Da zur Erfassung der mundgesundheitsbezogenen Lebensqualität von Personen mit kognitiver Einschränkung bislang keine Fragebögen existierten, wurde der OHIP-G5-Fragebogen (John 2004) in einfache Sprache übersetzt. Die Inhalte der Fragen des OHIP-G5 wurden hierzu in Kooperation mit dem Büro für Leichte Sprache ${ }^{1}$ in kurze Sätze mit einfachem Satzbau überführt. Die barrierefreie Verständlichkeit der Fragen wurde durch das Büro für Leichte Sprache mit Testpersonen mit kognitiver Einschränkung überprüft. Anschließend wurde der Fragebogen vom Netzwerk für Leichte Sprache und Barrierefreiheit mit dem Prüfsiegel „Inclusion Europe“ zertifiziert. Im Folgenden wird der Fragebogen als OHIP-Ge (Oral-Health-Impact-Profile-German-easy) bezeichnet. Die Fragen zur Wahrnehmung der MLQ konnten von den Patienten anhand von drei Abstufungen (,nein“ = Fragenwert: 4; „etwas“ = Fragenwert: 2; ,ja“ = Fragenwert: 0) beantwortet werden. Die fünfte Frage „Manchmal tut mir mein Mund weh“ galt es im Gegensatz zu den anderen Fragen zu verneinen, wenn keine Beschwerden im Mundraum bestanden. Allen Patienten wurden die Fragen des Fragebogens einzeln vorgelesen. Wenn ein Patient aufgrund der Schwere der kognitiven Einschränkung nicht auf die Fragen antworten konnte, wurde dies auf dem Fragebogen vermerkt. Die einzelnen Fragenwerte wurden zu dem OHIP-GeSummenwert (Minimum: 0 = keine Einschränkung der MLQ; Maximum: $20=$ maximale Einschränkung der MLQ) addiert (Tabelle 3-2).

Tabelle 3-2: Angepasster OHIP-G5-Fragebogen zur Selbstbefragung der Patienten in leichter Sprache, d. h. es wird bewusst auf Satzzeichen und komplizierte Formulierungen verzichtet. Die Itempunkte 0, 2 und 4 werden für die Auswertung zum Summenwert addiert. Der Maximalwert von 20 entspricht einer maximal eingschränkten MLQ.

\begin{tabular}{|l|c|c|c|}
\cline { 2 - 4 } \multicolumn{1}{c|}{} & ja & etwas & nein \\
\hline Vieles was ich esse schmeckt mir & 0 & 2 & 4 \\
\hline Meine Zähne sind schön & 0 & 2 & 4 \\
\hline Andere Menschen mögen meine Zähne & 0 & 2 & 4 \\
\hline Ich kann mit meinen Zähnen gut essen und kauen & 0 & 2 & 4 \\
\hline Manchmal tut mir mein Mund weh & 4 & 2 & 0 \\
\hline
\end{tabular}

\footnotetext{
${ }^{1}$ Büro für Leichte Sprache und Barrierefreiheit, Süntelstraße 32a, 49088 Osnabrück
} 


\subsection{Anamnese und Betreuungszeit}

Zur Beschreibung der Patientenklientel und zur Überprüfung behinderungsassoziierter, anamnestischer und demografischer Faktoren auf die MLQ wurden die in Tabelle 3-3 dargestellten Informationen der Aktendokumentation entnommenen. Außerdem wurde die tägliche durchschnittliche Betreuungszeit erfragt.

Tabelle 3-3: Erfassung potenzieller Einflussfaktoren auf die MLQ aus der Anamnese

\begin{tabular}{|l|l|}
\hline Geschlecht & männlich/weiblich \\
\hline Alter & in Jahren \\
\hline Wohnsituation & Wohneinrichtung, Familie oder allein \\
\hline Körperliche Behinderung & ja/nein \\
\hline $\begin{array}{l}\text { Ätiologie der kognitiven } \\
\text { Einschränkung }\end{array}$ & $\begin{array}{l}\text { angeboren, erworben oder } \\
\text { progredient }\end{array}$ \\
\hline $\begin{array}{l}\text { Ernährung ausschliesslich über } \\
\text { Ernährungssonde }\end{array}$ & ja/nein \\
\hline $\begin{array}{l}\text { Grund des Vorstellens in der } \\
\text { Klinik }\end{array}$ & $\begin{array}{l}\text { Schmerzen, Schwellung, Karies, Pro- } \\
\text { phylaxe oder sonstiges }\end{array}$ \\
\hline Betreuungszeit & Minuten pro Tag \\
\hline $\begin{array}{l}\text { *abgeleitet aus der medizinischen Diagnose, die als Grund der Behinderung erfragt } \\
\text { wurde }\end{array}$ \\
\hline
\end{tabular}

\subsection{Mundgesundheitsparameter}

Der Mundgesundheitszustand der Patienten wird durch die kariöse sowie parodontale Schädigung der Zähne und die vertikalen Abstützungszonen beschrieben. Die Mundgesundheitsparameter hierzu werden standardmäßig im Rahmen der ITN erhoben und in der Patientenakte dokumentiert.

\subsubsection{Karieserfahrung}

Der DMF-T-Index beschreibt kumulativ die Anzahl kariöser ( $D$ = Decayed), fehlender ( $M=$ Missing) bzw. mit einer Füllung versorgten ( $F=$ Filled) Zähne ( $T$ = Teeth) im bleibenden Gebiss (Klein et al. 1938). Er gilt als international anerkannte Maßzahl der Kariesprävalenz bzw. der Kariesinzidenz. Bei 28 Zähnen 
im bleibenden Gebiss (Weisheitszähne werden nicht berücksichtigt) ergibt sich maximal ein Wert von 28. Zähne mit suffizienten Füllungen (F), kariesfreie Zähne sowie fehlende bzw. aufgrund von Karies entfernte (M) und kariöse (D) Zähne wurden anhand der Aktendokumentation ermittelt und zu einem Summenwert (DMF-T) addiert.

\subsubsection{Parodontale Schädigung}

Der Paradontale Screening Index (PSI) dient der Ermittlung des parodontalen Gesundheitszustandes (Cutress et al. 1978; Ainamo et al. 1982; Wolf et al. 2004). Tabelle 3-4 fasst die mit dem PSI klassifizierten Befunde sowie die parodontalen Verdachtsdiagnosen und Therapieempfehlungen zusammen. Die Sondierungstiefen, auftretende Blutungen beim Sondieren sowie das Vorliegen von Zahnstein und insuffizienten Restaurationen werden für jeden Zahn erfasst. Pro Kiefersextant wird der höchste Codewert ermittelt. Im Rahmen der Studie wurden für jeden Patienten alle sechs PSI-Werte der Aktendokumentation entnommen.

Tabelle 3-4: Parodontaler Screening Index entsprechend der American Academy of Periodontology (AAP) und der American Dental Association (ADA).

\begin{tabular}{|c|c|c|c|c|c|}
\hline \multicolumn{6}{|c|}{ Befunde } \\
\hline Sondierungstiefen & $<3,5 \mathrm{~mm}$ & $\leq 3,5 \mathrm{~mm}$ & $\leq 3,5 \mathrm{~mm}$ & $\begin{array}{l}\text { zwischen } 3,5 \\
\text { und } 5,5 \mathrm{~mm}\end{array}$ & $>5,5 \mathrm{~mm}$ \\
\hline Blutung bei Sondierung & nein & ja & ja & möglich & möglich \\
\hline $\begin{array}{l}\text { Zahnstein/Plaque vor- } \\
\text { handen }\end{array}$ & nein & nein & ja & möglich & möglich \\
\hline $\begin{array}{l}\text { Restaurativer Behand- } \\
\text { lungsbedarf (insuffiziente } \\
\text { Restaurationen, kariöse } \\
\text { Läsionen) } \\
\end{array}$ & nein & nein & ja & möglich & möglich \\
\hline PSI-Code & 0 & 1 & 2 & 3 & 4 \\
\hline Verdachtsdiagnose & $\begin{array}{l}\text { parodontale } \\
\text { Gesundheit }\end{array}$ & Gingivitis & Gingivitis & Parodontitis & Parodontitis \\
\hline Therapieempfehlung & keine & $\begin{array}{l}\text { Instruktion zur } \\
\text { Verbesserung } \\
\text { der Mund- } \\
\text { Hygiene }\end{array}$ & $\begin{array}{l}\text { Instruktion zur } \\
\text { Verbesserung } \\
\text { der Mund- } \\
\text { hygiene, } \\
\text { professionelle } \\
\text { Zahnreinigung } \\
\text { (PZR) }\end{array}$ & $\begin{array}{c}\text { Instruktion zur } \\
\text { Verbesserung } \\
\text { der Mund- } \\
\text { hygiene, } \\
\text { professionelle } \\
\text { Zahnreinigung } \\
\text { (PZR), } \\
\text { Parodontal- } \\
\text { therapie (Deep } \\
\text { Scaling) } \\
\end{array}$ & \begin{tabular}{|} 
Instruktion zur \\
Verbesserung \\
der Mund- \\
hygiene, \\
professionelle \\
Zahnreinigung \\
(PZR), \\
Parodontal- \\
therapie \\
(Deep \\
Scaling) \\
\end{tabular} \\
\hline
\end{tabular}




\subsubsection{Vertikale Stützzonenverteilung}

In der vorliegenden Studie erfolgte die Einteilung der vertikalen Abstützung der Kiefer durch die Seitenzähne anhand des Bewertungsschemas nach Eichner (1990). Tabelle 3-5 zeigt die Einteilung nach der Anzahl der (noch) vorhandenen Stützzonen in drei Hauptgruppen und die verschiedenen Untergruppen. Entsprechend der Aktendokumentation wurden für jeden Patienten die Hauptund Untergruppen abgeleitet.

Tabelle 3-5: Vertikale Stützzonenverteilung nach Eichner (1990).

\begin{tabular}{|c|c|c|c|}
\hline \multicolumn{2}{|c|}{ Eichner-Hauptgruppe } & \multicolumn{2}{|c|}{ Eichner-Nebengruppen } \\
\hline \multirow{3}{*}{ A } & \multirow{3}{*}{$\begin{array}{l}\text { Alle vier Stützzonen weisen anta- } \\
\text { gonistischen Kontakt auf }\end{array}$} & A1 & Beide Kiefer vollständig bezahnt \\
\hline & & A2 & Ein Kiefer vollständig bezahnt, ein Kiefer mit zahnbegrenzten Lücken \\
\hline & & A3 & Beide Kiefer mit Lücken, Abstützung in allen vier Stützzonen \\
\hline \multirow{4}{*}{ B } & \multirow{4}{*}{$\begin{array}{l}\text { Fehlender antagonistischer Zahn- } \\
\text { kontakt }\end{array}$} & B1 & Antagonistischer Kontakt in drei Stützzonen \\
\hline & & B2 & Antagonistischer Kontakt in zwei Stützzonen \\
\hline & & B3 & Antagonistischer Kontakt in einer Stützzone \\
\hline & & B4 & $\begin{array}{l}\text { Antagonistischer Kontakt außerhalb der Stützzonen (im Frontzahnbe- } \\
\text { reich) }\end{array}$ \\
\hline \multirow{3}{*}{ C } & \multirow{3}{*}{$\begin{array}{l}\text { Weniger als vier Stützzonen weisen } \\
\text { antagonistischen Kontakt auf }\end{array}$} & C1 & Restzähne in beiden Kiefern \\
\hline & & C2 & Restzähne in einem Kiefer \\
\hline & & C3 & Keine Zähne \\
\hline
\end{tabular}




\subsection{Mundgesundheit vor der Therapie}

Zur Beschreibung des Mundgesundheitszustandes vor der Therapie und zur Überprüfung des Einflusses der Indizes auf die MLQ wurden die in Tabelle 3-6 aufgeführten Parameter der Aktendokumentation entnommen.

Tabelle 3-6: Mundgesundheitsparameter zur Beschreibung des Mundgesundheitszustandes vor der Therapie.

\begin{tabular}{|l|l|}
\hline Karieserfahrung und Sanierungszustand der Zähne \\
\hline DMF-T-Wert & $\begin{array}{l}\text { Summe aus der Anzahl an fehlenden, gefüllten und kariösen } \\
\text { Zähnen }\end{array}$ \\
\hline D-T-Wert & Anzahl kariöser Zähne \\
\hline M-T-Wert & Anzahl fehlender Zähne aufgrund von Karies \\
\hline F-T-Wert & Anzahl der Zähne mit suffizienten Füllungen \\
\hline DF-Wert & Summe aus der Anzahl an gefüllten und kariösen Zähnen \\
\hline Funktionswert & \\
\hline Gesamtanzahl der Zähne (0-28) \\
\hline Eichner-Funktionswert (A-C) \\
\hline Eichner-Funktionswert/Untergruppe (A1, A2, A3, B1, B2, B3, B4, C1, C2 oder C3) \\
\hline Parodontaler Gesundheitszustand [PSI] \\
\hline Parodontal gesund & Code 0 \\
\hline Gingivitis & Code 1 bis Code 2, maximal in einem Sextanten Code 3 \\
\hline Parodontitis & Code 3 und höher in mehr als einem Sextanten \\
\hline
\end{tabular}




\subsection{Therapiemaßnahmen und -umfang}

Zur Überprüfung der einzelnen Therapiemaßnahmen auf die MLQ wurden die in Tabelle 3-7 aufgeführten Informationen zu den im Rahmen der ITN durchgeführten Therapiemaßnahmen ebenfalls der Patientenakte entnommen.

Tabelle 3-7: Zahnmedizinische Therapiemaßnahmen und Therapieumfang.

\begin{tabular}{|c|c|}
\hline \multicolumn{2}{|l|}{ Parodontolale Maßnahmen } \\
\hline Professionelle Zahnreinigung & ja/nein \\
\hline Parodontitistherapie, Deep Scaling & ja/nein \\
\hline \multicolumn{2}{|l|}{ Endodontische Maßnahmen } \\
\hline Anzahl wurzelkanalbehandelter Zähne & $0-28$ \\
\hline \multicolumn{2}{|l|}{ Restaurative Maßnahmen } \\
\hline Anzahl an Füllungen (inklusive Weisheitszähne) & $0-32$ \\
\hline Anzahl an Füllungen (ohne Weisheitszähne) & $0-28$ \\
\hline \multicolumn{2}{|l|}{ Chirurgische Maßnahmen } \\
\hline Gesamtanzahl Extraktionen & $0-32$ \\
\hline Anzahl Extraktionen aufgrund parodontaler Destruktion & $0-32$ \\
\hline Anzahl Extraktionen aufgrund kariöser Destruktion & $0-32$ \\
\hline Anzahl Weisheitszahnextraktionen & $0-4$ \\
\hline Anzahl Extraktionen (ohne Weisheitszähne) & $0-28$ \\
\hline \multicolumn{2}{|l|}{ OP-Zeit } \\
\hline Dauer der Narkose & Minuten \\
\hline
\end{tabular}




\subsection{Mundgesundheit nach der Therapie}

Aus der Kombination der prätherapeutischen Befunde (Tabelle 3-6) und den im Rahmen der ITN erfolgten Therapiemaßnahmen (Tabelle 3-7) wurde der Mundgesundheitszustand für den Zeitpunkt nach der Therapie bestimmt. Die in Tabelle 3-8 dargestellten Mundgesundheitsparameter wurden zur Beschreibung des posttherapeutischen Mundgesundheitszustandes verwendet und als potenzielle Einflussfaktoren der MLQ überprüft.

Tabelle 3-8: Mundgesundheitsparameter zur Beschreibung des Mundgesundheitszustandes nach der Therapie. Berücksichtigt wurden alle bleibenden Zähne, ausgenommen der Weisheitszähne.

\begin{tabular}{|l|l|}
\hline \multicolumn{2}{|l|}{ Karieserfahrung und Sanierungszustand der Zähne } \\
\hline DMF-T-Wert & $\begin{array}{l}\text { Summe aus der Anzahl an fehlenden, gefüllten und kariösen } \\
\text { Zähnen }\end{array}$ \\
\hline D-T-Wert & Anzahl kariöser Zähne \\
\hline M-T-Wert & Anzahl aufgrund von Karies entfernter Zähne nach der Therapie \\
\hline F-T-Wert & Anzahl suffizient gefüllter Zähne nach der Therapie \\
\hline DF-Wert & Summe aus der Anzahl an gefüllten und kariösen Zähnen \\
\hline Funktionswert & $\begin{array}{l}\text { Aus Therapie resultierender Stützzonenverlust innerhalb der } \\
\text { Hauptklassifikation (A-C) nach Eichner (ja/nein) }\end{array}$ \\
\hline Stützzonenverlust \\
\hline Fehlende Zähne & $\begin{array}{l}\text { Anzahl aufgrund von Karies und/oder parodontaler Destruktion } \\
\text { entfernter Zähne nach der Therapie (0-28) }\end{array}$ \\
\hline Gesamtanzahl vorhandener Zähne (0-28) \\
\hline Eichner-Funktionswert (A-C) \\
\hline Eichner-Funktionswert (A1, A2, A3, B1, B2, B3, B4, C1, C2 oder C3) \\
\hline
\end{tabular}




\subsection{Statistische Analyse}

Für die statistische Analyse wurden die Daten anonymisiert. Es wurden nur die Patientendaten berücksichtigt, bei denen die Befragungen zur MLQ zu allen drei Zeitpunkten (T0, T1 und T2) erfolgte.

Die Summenwertveränderungen des OHIP-G14 und des OHIP-Ge im zeitlichen Verlauf (von T0 zu T1, von T1 zu T2 und von T0 zu T2) wurden mit nicht-parametrisch gemischten Regressionsanalysen analysiert. Zur Darstellung und Übersicht aller potenziellen Einflussfaktoren des OHIP-G14 und des OHIP-Ge wurden diese zunächst deskriptiv analysiert.

Mit einer univariaten logistischen Regression wurde der Einfluss von den in Tabelle 3-3 aufgelisteten anamnestischen Faktoren und den in Tabelle 3-6 aufgelisteten initialen Mundgesundheitsparametern auf die Summenwerte des OHIPG14 und des OHIP-Ge zum Zeitpunkt T0 überprüft. Die Variablen der Anamnese Tabelle 3-3 des Mundgesundheitszustandes vor und nach der Therapie (Tabelle 3-6 und Tabelle 3-8) und der erfolgten Therapiemaßnahmen (Tabelle 3-7) wurden außerdem als potenzielle Prädiktoren der OHIP-G14Summenwertveränderungen (von T0 zu T1, von T1 zu T2 und von T0 zu T2) mit einer univariaten logistischen Regression überprüft. Variablen mit $p<0,05$ wurden in eine multivariate Regressionsanalyse eingeschlossen. Um die Unabhängigkeit der Prädiktoren für die multivariate Regressionsanalyse zu überprüfen, wurden die Faktoren untereinander auf ihre Abhängigkeit untersucht. Hierzu wurden die Prädiktoren paarweise korreliert. Die Variablen, die mit keinem weiteren Faktor eine Korrelation von $r>0,8$ aufwiesen, wurden in das finale Modell eingeschlossen. Von den Faktoren, die eine paarweise Korrelation von $r>0,8$ aufwiesen, wurde ausschliesslich der Faktor mit dem geringeren $p$-Wert aus dem univariaten Modell in das finale Modell eingeschlossen.

Analog zur Analyse potenzieller Einflussfaktoren auf den OHIP-G14 wurden dieselben Variablen als Prädiktoren der OHIP-Ge-Summenwertveränderungen (von T0 zu T1, von T1 zu T2 und von T0 zu T2) mit einer univariaten logistischen Regression analysiert. Aufgrund der geringeren Fallzahl wurden bei dem OHIP-Ge Faktoren mit $p<0,1$ in die multivariate Regressionsanalyse eingeschlossen. 
Die Übereinstimmung zwischen Fremd- und Selbstbefragung wurde mittels Korrelationsanalyse nach Spearman berechnet, sofern Ergebnisse aus der Befragung des Betreuers (OHIP-G14) und aus der Befragung des Patienten selbst (OHIP-Ge) vorlagen $(n=26)$.

Mit dem Wilcoxon-Mann-Whitney-U-Test für unabhängige Stichproben wurde ein Vergleich der OHIP-G14-Summenwerte zum Zeitpunkt T1 der Gruppen, bei denen ausschließlich Summenwerte der Fremdbefragungen (OHIP-G14) und der Gruppe bei der Summenwerte aus Fremd- und Selbstbefragungen (OHIPG14 und OHIP-Ge) vorlagen, durchgeführt. Mit einer nicht-parametrischen Modellanalyse wurde die Übereinstimmung der OHIP-G14Summenwertveränderung dieser beiden Gruppen zwischen den Befragungszeitpunkten überprüft.

Das Signifikanzniveau wurde, sofern nicht anders beschrieben, auf $p<0,05$ festgelegt. Die Auswertungen wurden in Zusammenarbeit mit dem Institut für Medizinische Statistik der Universitätsmedizin Göttingen mit der Statistiksoftware R (version 3.4.0; R Core Team 2018) durchgeführt. Für die ordinalen und nicht-parametrischen gemischten Modelle wurden die R-Pakete „ordinal“ beziehungsweise „nparLD“ verwendet. 


\section{$4 \quad$ Ergebnisse}

Während des Studienzeitraumes entsprachen 77 Patienten den Einschlusskriterien der vorliegenden Studie. Davon willigten 61 gesetzliche Betreuer in die Studienteilnahme ein, woraus sich ein Studienkollektiv von $\mathrm{n}=61$ Patienten ergab. Sieben Patienten nahmen den Termin zur zahnmedizinischen Therapie in Vollnarkose, z. B. aufgrund eines schlechten Allgemeinzustands, nicht wahr. Je ein Patient und dessen Betreuer konnten zu T1 bzw. zu T2 nicht befragt werden, weil ein Betreuer weitere Kontrolluntersuchungen ablehnte und eine Patientin zwischenzeitlich verstorben war. Insgesamt wurde die mundgesundheitsbezogene Lebensqualität von 52 Patienten (38 $\pm 13,5$ Jahre, $51 \%$ weiblich) zu allen drei Zeitpunkten (T0, T1 und T2) erfasst. Die Patienten unterschieden sich in ihrer Fähigkeit zur Kommunikation: 27 Patienten $(37,3 \pm 13$ Jahre, 44,4\% weiblich) konnten auf die Fragen des OHIP-Ge antworten. Bei 25 Patienten konnte die MLQ ausschließlich durch die Befragung der Betreuer (OHIP-G14) bestimmt werden, da die Patienten nicht auf den OHIP-Ge antworteten. Ein Patient wurde ausschließlich selbst befragt, da dieser sich zwar in einem gesetzlichen Betreuungsverhältnis befand, im Alltag allerdings nicht täglich von einer Betreuungsperson unterstützt wurde. Bei 26 Personen konnte die $M L Q$ sowohl über die Fremd- als auch über die Selbstbefragung erfasst werden. Abbildung 4-1 zeigt den beschriebenen Studienablauf, mit Bezug auf die Teilnahme der Patienten und deren Betreuungspersonen. 


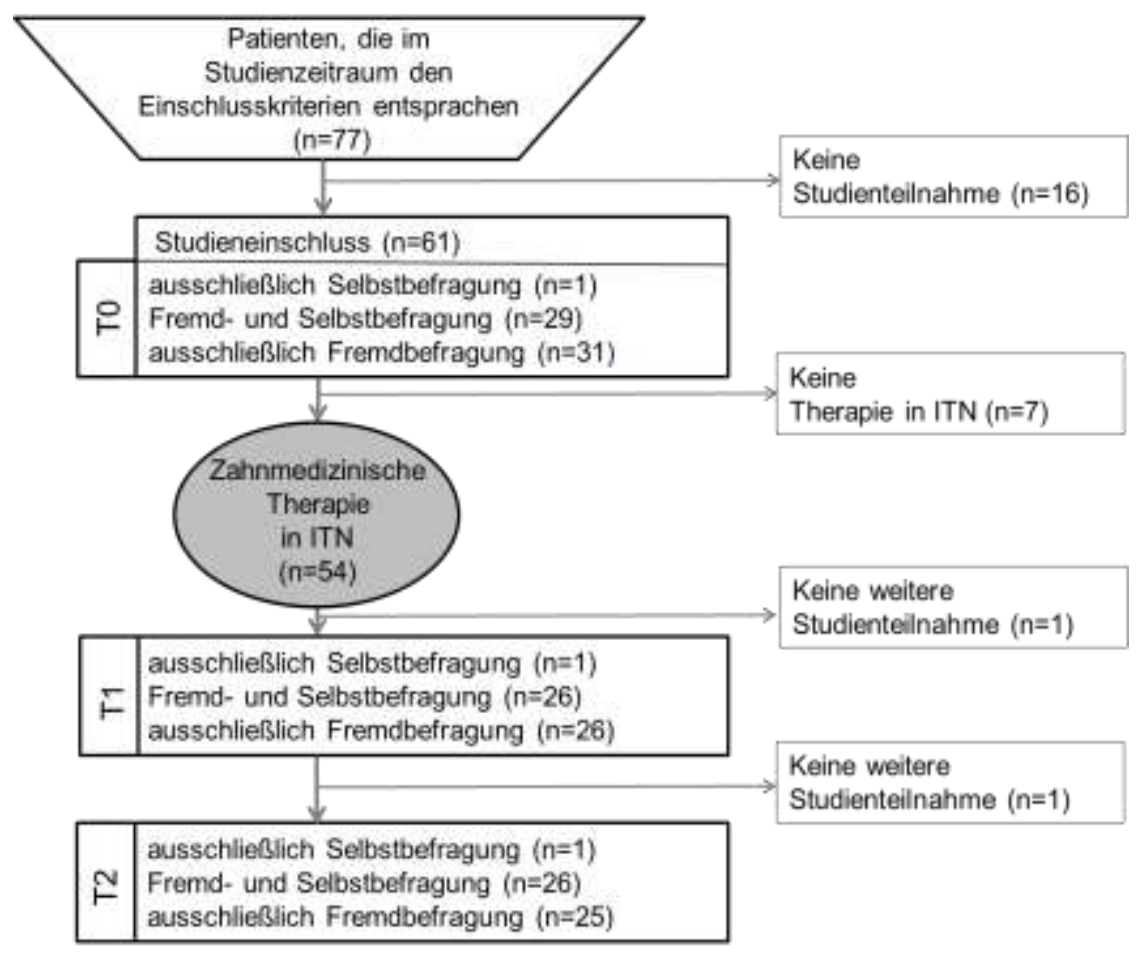

Abbildung 4-1: Studienablauf und Anzahl teilnehmender Patienten bzw. Betreuer.

\subsection{Mundgesundheitsbezogene Lebensqualität}

In der Fremd- und in der Selbstberfragung wurden nach der Therapie in ITN weniger Einschränkungen der MLQ angegeben. Paarvergleiche der OHIP-G14-Summenwerte (Fremdbefragung) ergaben eine signifikante Reduktion von T0 zu T1, T0 zu T2 und T1 zu T2 ( $<<0,001)$ (Abbildung 4-2). Die OHIPGe-Summenwerte (Selbstbefragung) reduzierten sich ebenfalls signifikant von T0 zu T1 ( $p<0,023)$, von T0 zu T2 $(p<0,001)$ und von T1 zu T2 $(p<0,001)$ (Abbildung 4-3). Zwischen den OHIP-G14-Summenwerten der Gruppe, die ausschließlich fremdbefragt (nur OHIP-G14) wurden, und den OHIP-G14Summenwerten der Gruppe, die zusätzlich zur Fremdbefragung auch selbstbefragt (OHIP-G14 und OHIP-Ge) werden konnte, bestand zum Zeitpunkt T0 kein Unterschied. Im Verlauf reduzierte sich der OHIP-G14-Summenwert der Grup$p e$, die zusätzlich selbstbefragt werden konnte, signifikant schwächer $(p<$ 0,003 ) als bei der Gruppe, die ausschließlich fremdbefragt wurde (Abbildung 4-4). 


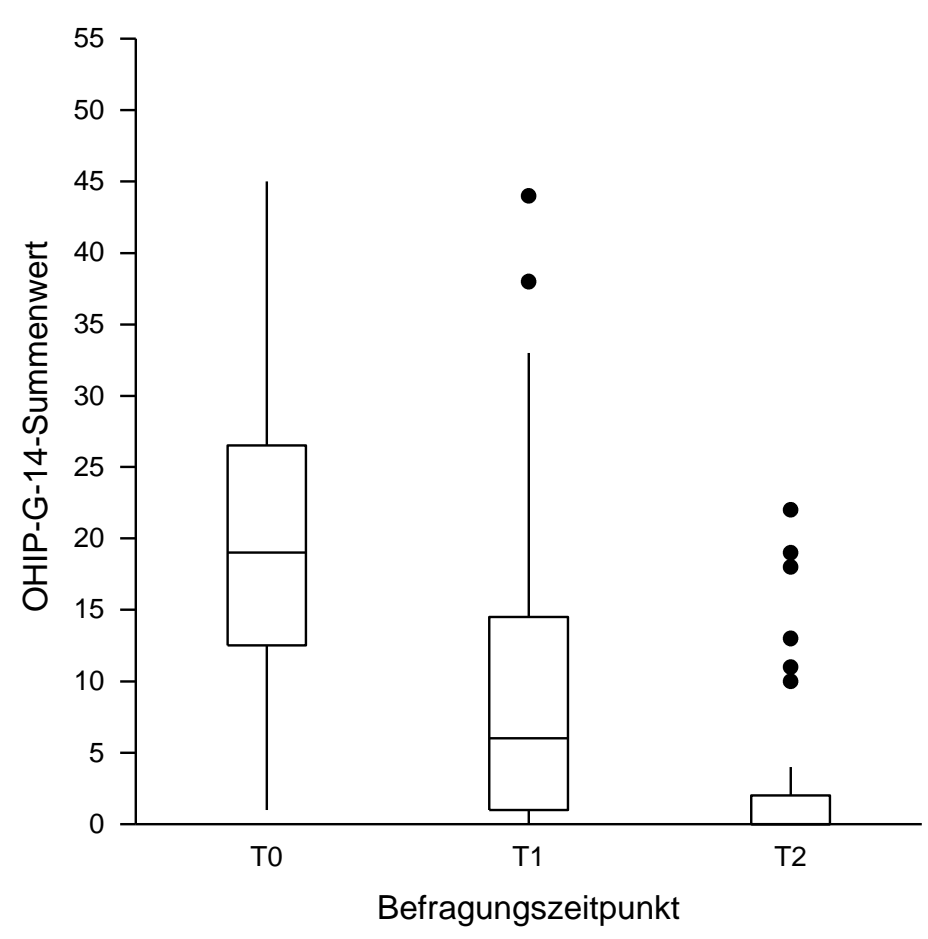

Abbildung 4-2: Summenwerte des OHIP-G14 (Median, Quartile und Ausreißer) zu den drei Befragungszeitpunkten T0, T1 und T2.

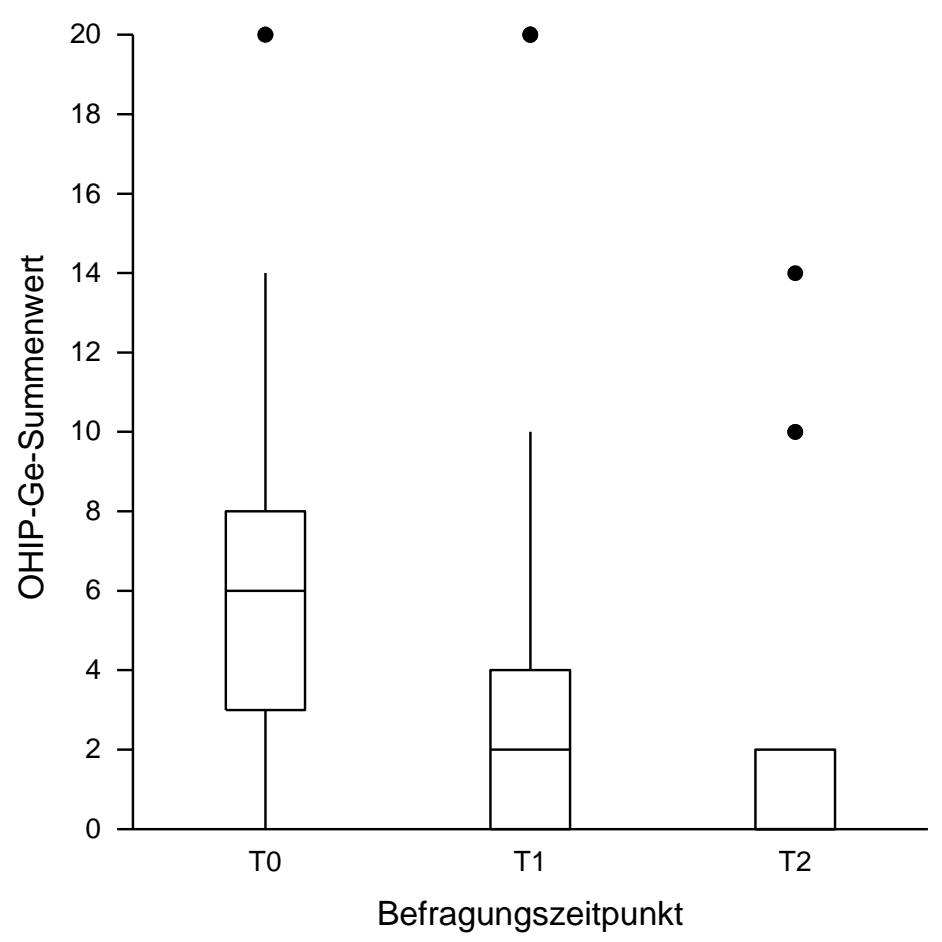

Abbildung 4-3: Summenwerte des OHIP-Ge (Median, Quartile und Ausreißer) zu den drei Befragungszeitpunkten T0, T1 und T2. 


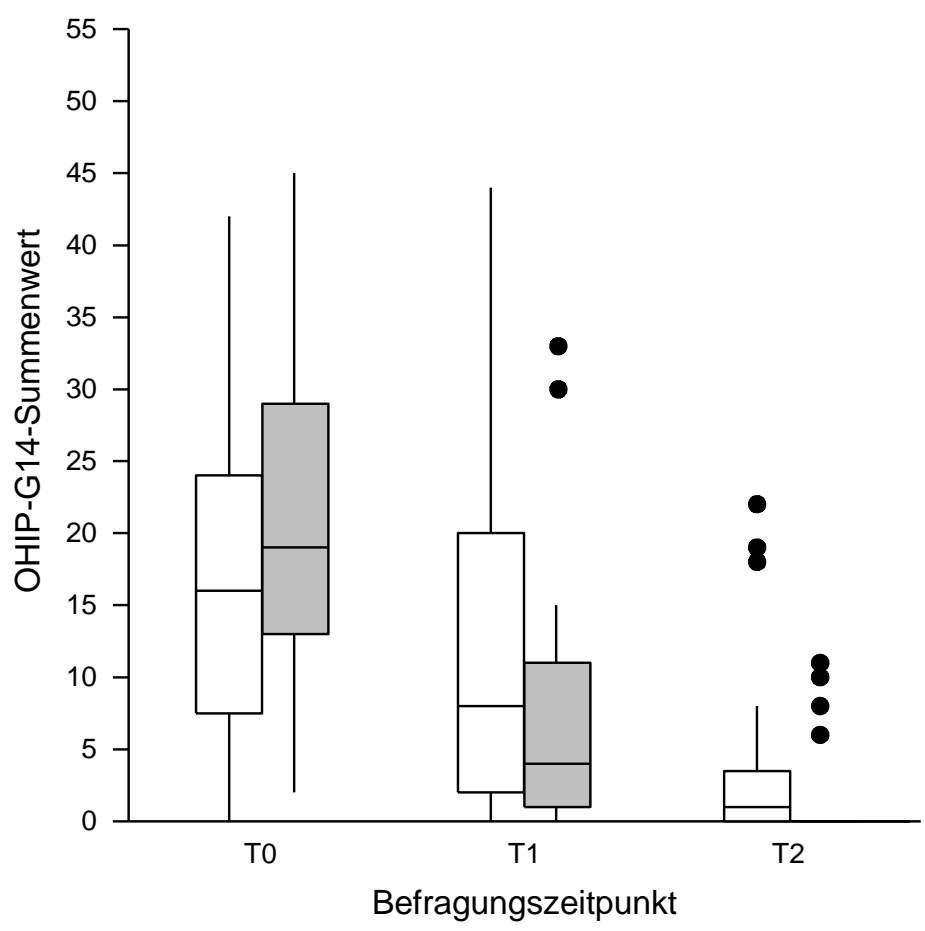

Abbildung 4-4: Summenwerte des OHIP-G14 (Median, Quartile und Ausreißer) von Patienten mit Selbstbefragung (weisse Balken) und Patienten ohne Selbstbefragung (graue Balken) zu den drei Befragungszeitpunkten T0, T1 und T2.

\subsubsection{Antwortenverteilung der Fremdbefragung}

Die Antworthäufigkeiten zu den einzelnen Items des OHIP-G14 sind in Tabelle 4-1 dargestellt. 
Tabelle 4-1: Antworten der Fremdbefragung ( $\mathrm{n}=$ Anzahl der Antworten), Punktwert pro Item (Mittelwert \pm Standardabweichung) und Summenwert (Mittelwert \pm Standardabweichung) des OHIP-G14 zu den Zeitpunkten T0, T1 und T2. Hauptsächlich war die MLQ zu den Zeitpunkten T0 und T1 durch Schmerzen (rot) eingeschränkt. Kein Item wurde zum Zeitpunkt T2 schlechter bewertet als zum Zeitpunkt T1 oder T0.

\begin{tabular}{|c|c|c|c|c|c|c|c|c|c|c|c|c|c|c|c|c|c|c|}
\hline & \multicolumn{6}{|c|}{ T0 } & \multicolumn{6}{|c|}{ T1 } & \multicolumn{6}{|c|}{ T2 } \\
\hline OHIP-G14-Item & $\begin{array}{l}\stackrel{\oplus}{\epsilon} \\
\text { (0) } \\
\end{array}$ & 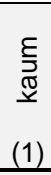 & 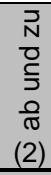 & (3) & $\begin{array}{l}\text { 픙 } \\
\text { ㄹ } \\
0 \\
\text { D } \\
\text { (4) }\end{array}$ & Punktwert/Item & $\begin{array}{l}. \stackrel{\oplus}{ᄃ} \\
(0) \\
\end{array}$ & $\begin{array}{l}\underline{E} \\
\underline{\widetilde{\sigma}} \\
\underline{\underline{y}} \\
\text { (1) }\end{array}$ & 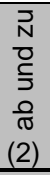 & Е & $\begin{array}{l}\bar{T} \\
\text { ㅇ } \\
\frac{\bar{D}}{0} \\
\infty \\
(4) \\
\end{array}$ & Punktwert/ltem & $\begin{array}{l}\stackrel{\oplus}{\leftrightarrows} \\
\text { (0) } \\
\end{array}$ & 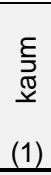 & 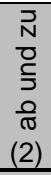 & (3) & 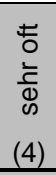 & Punktwert//tem \\
\hline Schwierigkeiten, Worte auszusprechen & 35 & 4 & 6 & 3 & 3 & $0,7 \pm 1,2$ & 39 & 6 & 2 & 1 & 3 & $0,5 \pm 1,1$ & 45 & 5 & 0 & 0 & 1 & $0,2 \pm 0,6$ \\
\hline Beeinträchtigung des Geschmackssinns & 35 & 7 & 5 & 3 & 1 & $0,6 \pm 1,0$ & 36 & 5 & 6 & 2 & 2 & $0,6 \pm 1,1$ & 47 & 2 & 2 & 0 & 0 & $0,1 \pm 0,4$ \\
\hline Schmerzen im Mundbereich & 2 & 4 & 18 & 16 & 11 & $2,6 \pm 1,0$ & 18 & 16 & 12 & 3 & 2 & $1,1 \pm 1,1$ & 47 & 1 & 3 & 0 & 0 & $0,1 \pm 0,5$ \\
\hline Schmerzen beim Essen & 21 & 12 & 4 & 7 & 7 & $1,4 \pm 1,5$ & 27 & 13 & 7 & 3 & 1 & $0,8 \pm 1,0$ & 41 & 6 & 4 & 0 & 0 & $0,3 \pm 0,6$ \\
\hline Verlegen & 21 & 9 & 14 & 5 & 2 & $1,2 \pm 1,2$ & 32 & 10 & 6 & 1 & 2 & $0,6 \pm 1,0$ & 43 & 4 & 4 & 0 & 0 & $0,2 \pm 0,6$ \\
\hline Angespannt & 11 & 8 & 15 & 7 & 10 & $1,9 \pm 1,4$ & 23 & 12 & 9 & 3 & 4 & $1,1 \pm 1,3$ & 41 & 7 & 2 & 1 & 0 & $0,3 \pm 0,6$ \\
\hline Unbefriedigende Ernährung & 26 & 9 & 5 & 5 & 6 & $1,1 \pm 1,4$ & 36 & 7 & 2 & 5 & 1 & $0,6 \pm 1,1$ & 44 & 4 & 2 & 0 & 1 & $0,2 \pm 0,7$ \\
\hline Mahlzeiten unterbrechen & 27 & 7 & 5 & 5 & 7 & $1,2 \pm 1,5$ & 29 & 11 & 7 & 2 & 2 & $0,8 \pm 1,1$ & 42 & 7 & 1 & 1 & 0 & $0,2 \pm 0,6$ \\
\hline Schwierigkeiten zu entspannen & 13 & 8 & 15 & 8 & 7 & $1,8 \pm 1,4$ & 28 & 8 & 7 & 6 & 2 & $0,9 \pm 1,2$ & 46 & 0 & 5 & 0 & 0 & $0,2 \pm 0,6$ \\
\hline Unsicherer Umgang mit anderen & 34 & 9 & 2 & 5 & 1 & $0,6 \pm 1,1$ & 40 & 7 & 2 & 2 & 0 & $0,3 \pm 0,7$ & 48 & 0 & 3 & 0 & 0 & $0,1 \pm 0,5$ \\
\hline Reizbarer anderen Menschen gegenüber & 15 & 12 & 10 & 10 & 4 & $1,5 \pm 1,3$ & 33 & 8 & 4 & 4 & 2 & $0,7 \pm 1,2$ & 47 & 4 & 0 & 0 & 0 & $0,1 \pm 0,3$ \\
\hline Antriebsschwächer im Alltag & 14 & 12 & 12 & 9 & 4 & $1,5 \pm 1,3$ & 32 & 10 & 4 & 3 & 2 & $0,7 \pm 1,1$ & 44 & 3 & 4 & 0 & 0 & $0,2 \pm 0,6$ \\
\hline Weniger zufrieden im Alltag & 14 & 8 & 14 & 10 & 5 & $1,7 \pm 1,3$ & 27 & 13 & 4 & 6 & 1 & $0,8 \pm 1,1$ & 44 & 3 & 2 & 1 & 1 & $0,3 \pm 0,8$ \\
\hline Generell anderes Verhalten im Alltag & 22 & 13 & 7 & 4 & 5 & $1,2 \pm 1,3$ & 34 & 9 & 6 & 1 & 1 & $0,5 \pm 0,9$ & 47 & 1 & 1 & 2 & 0 & $0,2 \pm 0,1$ \\
\hline Summenwert OHIP-G14 & & & & 18,8 & \pm 11 & & & & & 10,1 & \pm 11 & & & & & & $\pm 5,3$ & \\
\hline
\end{tabular}




\subsubsection{Antwortenverteilung der Selbstbefragung}

Die Antworthäufigkeiten zu den einzelnen Items des OHIP-Ge sind in Tabelle 4-2 dargestellt. Alle Items wurden zum T1 und T2 besser bewertet als zu T0.

Tabelle 4-2: Antworten der Selbtbefragung ( $\mathrm{n}=$ Anzahl der Antworten), Punktwert pro Item (Mittelwert \pm Standardabweichung) und Summenwert (Mittelwert \pm Standardabweichung) des OHIPGe zu den Zeitpunkten T0, T1 und T2. Hauptsächlich war die MLQ zu den Zeitpunkten T0 und zu T1 durch Schmerzen (rot) eingeschränkt.

\begin{tabular}{|c|c|c|c|c|c|c|c|c|c|c|c|c|}
\hline & \multicolumn{4}{|c|}{ TO } & \multicolumn{4}{|c|}{ T1 } & \multicolumn{4}{|c|}{ T2 } \\
\hline & $\begin{array}{c}. \underline{\text { D }} \\
(0 / 4)\end{array}$ & $\begin{array}{l}\stackrel{0}{\mathbb{N}} \\
\sum_{\mathbb{0}}^{\mathbb{2}} \\
\text { (2) }\end{array}$ & $\begin{array}{l}\cdot \stackrel{\frac{\subseteq}{\Phi}}{\subseteq} \\
(4 / 0)\end{array}$ & $\begin{array}{l}\text { Punkt- } \\
\text { wert/ } \\
\text { Item }\end{array}$ & $\begin{array}{c}\text {. } \\
(0 / 4)\end{array}$ & $\begin{array}{l}\frac{9}{\pi} \\
\sum_{0}^{2} \\
\text { (2) }\end{array}$ & $\begin{array}{l}\stackrel{\cdot \frac{c}{\Phi}}{\subseteq} \\
(4 / 0)\end{array}$ & $\begin{array}{l}\text { Punkt- } \\
\text { wert/ } \\
\text { Item }\end{array}$ & $\begin{array}{c}. \underline{\text { 口 }} \\
(0 / 4)\end{array}$ & $\begin{array}{l}\stackrel{9}{\pi} \\
\sum_{0}^{\mathbb{Z}} \\
\text { (2) }\end{array}$ & $\begin{array}{l}\stackrel{\complement}{\Phi} \\
(4 / 0)\end{array}$ & $\begin{array}{l}\text { Punkt- } \\
\text { wert/ } \\
\text { Item }\end{array}$ \\
\hline $\begin{array}{l}\text { Vieles was ich esse } \\
\text { schmeckt mir }\end{array}$ & 19 & 2 & 6 & $1,0 \pm 1,7$ & 21 & 3 & 3 & $0,7 \pm 1,4$ & 23 & 1 & 3 & $0,5 \pm 1,3$ \\
\hline $\begin{array}{l}\text { Manchmal tut mir mein } \\
\text { Mund weh }\end{array}$ & 13 & 4 & 10 & $2,2 \pm 1,9$ & 6 & 7 & 14 & $1,4 \pm 1,6$ & 1 & 2 & 24 & $0,3 \pm 0,9$ \\
\hline Meine Zähne sind schön & 18 & 3 & 6 & $1,1 \pm 1,7$ & 20 & 2 & 5 & $0,9 \pm 1,6$ & 25 & 0 & 2 & $0,3 \pm 1,1$ \\
\hline $\begin{array}{l}\text { Ich kann mit meinen Zäh- } \\
\text { nen gut essen und kauen }\end{array}$ & 25 & 1 & 1 & $0,2 \pm 0,8$ & 25 & 0 & 2 & $0,3 \pm 1,1$ & 26 & 1 & 0 & $0,1 \pm 0,8$ \\
\hline $\begin{array}{l}\text { Andere Menschen mögen } \\
\text { meine Zähne }\end{array}$ & 15 & 8 & 4 & $1,2 \pm 1,5$ & 17 & 6 & 4 & $1,0 \pm 1,5$ & 19 & 6 & 2 & $0,7 \pm 1,3$ \\
\hline Summenwert OHIP-Ge & \multicolumn{4}{|c|}{$5,8 \pm 4,6$} & \multicolumn{4}{|c|}{$4,3 \pm 5,9$} & \multicolumn{4}{|c|}{$2,0 \pm 3,9$} \\
\hline
\end{tabular}

\subsection{Korrelation von Selbst- und Fremdbefragung}

Tabelle 4-3 zeigt, dass die Summenwerte des OHIP-Ge (Selbstbefragung) und die Summenwerte des OHIP-G14 (Fremdbefragung) moderat korreliert waren. Die tägliche Betreuungszeit hatte auf die Korrelation keinen signifikanten Einfluss.

Tabelle 4-3: Spearman Korrelationskoeffizienten ( $r$ ) der Betreuer- und Patientenbefragung über alle Betreuungszeiten

\begin{tabular}{|l|l|l|}
\hline Vergleich der Summenwerte OHIP-Ge/OHIP-G14 & Spearman, $\mathbf{r}$ & $\mathbf{p}$-Wert \\
\hline Gesamt & 0,43 & $<0,001$ \\
\hline Betreuungszeit $>10$ Stunden/Tag & 0,49 & 0,001 \\
\hline Betreuungszeit < 10 Stunden/ Tag & 0,52 & 0,001 \\
\hline
\end{tabular}




\subsection{Patientenkollektiv}

Das Patientenkollektiv war in Bezug auf die Kommunikationsfähigkeit heterogen. Fünfundzwanzig Patienten (48,08\%) zeigten sich bei der Selbstbefragung fremd- und/oder autoagressiv oder reagierten gar nicht auf die Befragung mit dem OHIP-Ge. Siebenundzwanzig Patienten (51,92\%) beantworteten bei allen drei Befragungszeitpunkten jeweils alle Items des OHIP-Ge. Die deskriptive Analyse der demografischen Merkmale bei den Patienten mit Fremdbefragung und bei den Patienten mit Selbstbefragung ergab keine stichprobenrelevanten Unterschiede. Die Geschlechterverteilung und das Alter der Patienten waren ähnlich (Tabelle 4-4).

Tabelle 4-4: Demografische Merkmale der Patientenklientel

\begin{tabular}{|l|c|c|}
\cline { 2 - 3 } \multicolumn{1}{c|}{} & $\begin{array}{c}\text { Fremdbefragung } \\
\mathrm{n}=51(100 \%)\end{array}$ & $\begin{array}{c}\text { Selbstbefragung } \\
\mathrm{n}=27(100 \%)\end{array}$ \\
\hline weiblich & $26(51,0 \%)$ & $12(44,4 \%)$ \\
\hline $\begin{array}{l}\text { Alter zum Zeitpunkt der } \\
\text { ITN }\end{array}$ & $38 \pm 13,5$ Jahre & $37,3 \pm 13,0$ Jahre \\
\hline jüngster Patient & 18 Jahre & 19 Jahre \\
\hline ältester Patient & 67 Jahre & 61 Jahre \\
\hline
\end{tabular}

Die Ursachen für die kognitive Einschränkung aller Patienten waren sehr unterschiedlich. Bei $24(47,1 \%)$ Patienten bestand die kognitive Einschränkung aufgrund einer pränatalen Schädigung oder einer syndromalen Erkrankung (z. B. Trisomie 21, infantile Cerebralparese, fetales Alkoholsyndrom, Cri-DuChat-Syndrom) bereits seit der Geburt. Bei 16 (31,4 \%) Patienten kam es durch ein Ereignis in der Kindheit oder im Erwachsenenalter (z. B. Schädelhirntrauma oder Schlaganfall) zu einer Reduktion der kognitiven Leistungsfähigkeit. Bei zwölf $(21,6 \%)$ Patienten reduziert sich die kognitive Leistungsfähigkeit durch eine neurodegenerative Erkrankung (z. B. Neuromyelitis optica, Demenz) stetig. Nur bei fünf Patienten (9,8 \%) gaben die Betreuungspersonen an, dass zusätzlich zu der kognitiven Einschränkung keine körperlichen Einschränkungen bestanden. Die meisten Patienten waren in der Lage ohne Einschränkungen zu essen, allerdings wurden 17 (33,3\%) Patienten ausschließlich über eine Sonde 
ernährt. Siebenundzwanzig (52,9 \%) Patienten erhielten Hilfe bei der Durchführung der täglichen Zahnpflege. Bei neun (17,6\%) Patienten gaben die Betreuungspersonen an, dass die Durchführung von häuslichen Mundhygienemaßnahmen nicht möglich sei. Zur Kompensation behinderungsbedingter Defizite wurden die Patienten täglich in unterschiedlichem zeitlichen Umfang (729,4 \pm 483,2 Minuten) betreut. Einundfünfzig Prozent der erwachsenen Patienten lebten bei ihren Eltern, $47 \%$ lebten in einem Wohn- oder Pflegeheim und zwei Patienten lebten allein. Gründe für das Vorstellen in der Klinik zum Zeitpunkt T0 waren bei 35,3\% der Patienten Schmerzen, bei 15,7\% der Patienten wurden Schwellungen im Gesicht bemerkt und bei 21,6 \% der Patienten befürchtete die Betreuungsperson, dass Karies bestehen könnte.

\subsection{Mundgesundheit vor und nach der Therapie}

Der DMF-T betrug vor und nach der Therapie 13,7 $\pm 7,3$. Die Anzahl kariöser Zähne (D-T-Index) sank von 6,7 $\pm 5,9$ auf null. Die Anzahl fehlender Zähne (MT-Index) pro Patient stieg von 3,3 \pm 4,0 vor der Therapie auf 5,6 $\pm 6,4$ nach der Therapie. Außerdem stieg die Anzahl der gefüllten Zähne (F-T-Index) von 3,7 \pm 4,0 auf 8,1 $\pm 5,3$ (Tabelle 4-5).

Die parodontale Gesundheit war vor der Therapie gesamtheitlich schlecht. Die große Mehrheit der Patienten ( $n=48,92,4 \%$ ) wies eine Parodontitis (PSI-Code 3 oder 4 in zwei oder mehr Sextanten) auf. Die übrigen 3,9\% $(n=3)$ der Patienten hatten eine Gingivitis (PSI-Code 1-2, Code 3 in max. einem Sextanten). Bei einem Patienten (1,9\%) waren keine Angaben zur parodontalen Gesundheit verfügbar. 
Tabelle 4-5: Karieserfahrung (DMF-T) vor und nach der Therapie in ITN ( $\mathrm{n}=$ Anzahl der Patienten mit der jeweiligen Merkmalsausprägung, $\%=$ prozentualer Anteil der Patienten mit der jeweiligen Merkmalsausprägung, MW = Mittelwert, SD = Standardabweichung).

\begin{tabular}{|c|c|c|c|c|}
\hline & \multicolumn{2}{|c|}{ Vor der Therapie } & \multicolumn{2}{|c|}{ Nach der Therapie } \\
\hline & n (\%) & $M W \pm S D$ & n (\%) & $\mathrm{MW} \pm \mathrm{SD}$ \\
\hline DMF-T: & & $13,7 \pm 7,3$ & & $13,7 \pm 7,3$ \\
\hline 0 & 0 & & 0 & \\
\hline $1-9$ & $17(32,6)$ & & $17(32,6)$ & \\
\hline $10-19$ & $23(44,2)$ & & $23(44,2)$ & \\
\hline$\geq 20$ & $12(23,1)$ & & $12(23,1)$ & \\
\hline D-T: & & $6,7 \pm 5,9$ & & 0 \\
\hline 0 & $5(9,6)$ & & 0 & \\
\hline $1-9$ & $35(67,3)$ & & 0 & \\
\hline $10-19$ & $8(15,4)$ & & 0 & \\
\hline$\geq 20$ & $4(7,7)$ & & 0 & \\
\hline M-T: & & $3,3 \pm 4,0$ & & $5,6 \pm 6,4$ \\
\hline 0 & $11(21,2)$ & & $6(11,5)$ & \\
\hline $1-9$ & $37(71,2)$ & & $37(71,2)$ & \\
\hline $10-19$ & $4(7,7)$ & & $6(11,5)$ & \\
\hline$\geq 20$ & 0 & & $3(5,8)$ & \\
\hline F-T & & $3,7 \pm 4,0$ & & $8,1 \pm 5,3$ \\
\hline 0 & $14(26,9)$ & & $4(7,7)$ & \\
\hline $1-9$ & $33(63,5)$ & & $29(55,8)$ & \\
\hline $10-19$ & $5(9,6)$ & & $16(30,8)$ & \\
\hline$\geq 20$ & 0 & & $3(5,8)$ & \\
\hline
\end{tabular}

Die Zahnanzahl nach Therapie reichte von vollbezahnt (28 Zähne) bis zahnlos (0 Zähne). Im Durchschnitt betrug die Zahnanzahl (inklusive der Weisheitszähne) vor der Therapie 24,7 \pm 4,0 und sank durch die Therapie auf 21,5 \pm 6,8 Zähne pro Patient (Tabelle 4-6). 
Tabelle 4-6: Zahnanzahl der Patienten vor und nach der Therapie $(\mathrm{n}=$ Anzahl der Patienten mit der jeweiligen Merkmalsausprägung, $\%=$ prozentualer Anteil der Patienten mit der jeweiligen Merkmalsausprägung, $\mathrm{MW}=$ Mittelwert, $\mathrm{SD}=$ Standardabweichung).

\begin{tabular}{|c|c|c|c|c|}
\hline & \multicolumn{2}{|c|}{ Vor der Therapie } & \multicolumn{2}{|c|}{ Nach der Therapie } \\
\hline & n (\%) & $M W \pm S D$ & n (\%) & $M W \pm S D$ \\
\hline Zahnanzahl: & & $24,7 \pm 4,0$ & & $21,5 \pm 6,8$ \\
\hline 28 & $11(21,2)$ & & $6(11,5)$ & \\
\hline $20-27$ & $37(71,2)$ & & $32(61,5)$ & \\
\hline $10-19$ & $3(5,8)$ & & $10(19,2)$ & \\
\hline $1-9$ & $1(1,9)$ & & $2(3,8)$ & \\
\hline 0 & 0 & & $2(3,8)$ & \\
\hline
\end{tabular}

Tabelle 4-7 zeigt die Verteilungen der vertikalen Stützzonen vor und nach der Therapie. Eine intakte vertikale Abstützung mit antagonistischem Zahnkontakt in allen vier Kiefern lag vor der Therapie bei $84,6 \%$ der Patienten vor. Durch die Zahnextraktionen im Rahmen der Therapie kam es bei 24 (46,2 \%) Patienten zu dem Verlust von einer oder mehreren Stützzonen. Nach der Therapie war die vertikale Abstützung bei 32,7 \% der Patienten reduziert, und 9,6 \% aller Patienten hatten keine vertikale Abstützung mehr. 
Tabelle 4-7: Vertikale Stützzonenverteilung nach Eichner (1900) $(\mathrm{n}=$ Anzahl der Patienten mit der jeweiligen Merkmalsausprägung, $\%=$ prozentualer Anteil der Patienten mit der jeweiligen Merkmalsausprägung).

\begin{tabular}{|c|c|c|}
\hline & $\begin{array}{c}\text { Vor der } \\
\text { Therapie }\end{array}$ & $\begin{array}{l}\text { Nach der } \\
\text { Therapie }\end{array}$ \\
\hline & \multicolumn{2}{|c|}{ n (\%) } \\
\hline $\begin{array}{l}\text { A: Antagonistischer Kontakt in allen Stützzo- } \\
\text { nen }\end{array}$ & $44(84,6)$ & $30(57,7)$ \\
\hline $\begin{array}{l}\text { A1: Beide Kiefer vollbezahnt, alle Zähne wieder } \\
\text { aufbaufähig }\end{array}$ & $14(26,9)$ & $11(21,2)$ \\
\hline $\begin{array}{l}\text { A2: Ein Kiefer vollbezahnt, ein Kiefer mit zahnbe- } \\
\text { grenzten Lücken }\end{array}$ & $18(34,6)$ & $8(15,4)$ \\
\hline $\begin{array}{l}\text { A3: Beide Kiefer mit Lücken, Abstützung in allen } \\
\text { vier Stützzonen }\end{array}$ & $12(23,1)$ & $11(21,2)$ \\
\hline $\begin{array}{l}\text { B: Antagonistischer Kontakt nicht in allen } \\
\text { Stützzonen }\end{array}$ & $7(13,5)$ & $17(32,7)$ \\
\hline B1: Antagonistischer Kontakt in drei Stützzonen & $2(3,8)$ & $9(17,3)$ \\
\hline B2: Antagonistischer Kontakt in zwei Stützzonen & $4(7,7)$ & $6(11,5)$ \\
\hline B3: Antagonischer Kontakt in einer Stützzone & 0 & $2(3,8)$ \\
\hline $\begin{array}{l}\text { B4: Antagonistischer Kontakt außerhalb der } \\
\text { Stützzonen (im Frontzahnbereich) }\end{array}$ & $1(1,9)$ & 0 \\
\hline C: Kein antagonistischer Kontakt & $1(1,9)$ & $5(9,6)$ \\
\hline C1: Restzähne in beiden Kiefern & $1(1,9)$ & $3(5,8)$ \\
\hline C2: Restzähne in einem Kiefer & 0 & 0 \\
\hline C3: zahnlos & 0 & $2(3,8)$ \\
\hline
\end{tabular}

\subsection{Umfang der zahnmedizinischen Therapiemaßnahmen in ITN}

Die Therapiemaßnahmen setzten sich aus Zahnentfernungen, Füllungstherapien sowie Parodontalbehandlungen zusammen (Tabelle 4-8). Durchschnittlich wurden 3,1 \pm 4,1 Zähne pro Patient extrahiert und 4,7 \pm 4,4 Zähne pro Patient gefüllt. Bei 90 \% der Patienten erfolgte ein Deep Scaling (Parodontitistherapie). Ein Patient erhielt eine Wurzelkanalbehandlung. 
Tabelle 4-8: Therapiemaßnahmen während der ITN $\left({ }^{*}=\right.$ Weisheitszähne nicht berücksichtigt, $\mathrm{n}=$ Anzahl der Patienten mit der jeweiligen Merkmalsausprägung, $\%=$ prozentualer Anteil der Patienten mit der jeweiligen Merkmalsausprägung, $M W=$ Mittelwert, $S D=$ Standardabweichung).

\begin{tabular}{|c|c|c|}
\hline & n (\%) & $M W \pm S D$ \\
\hline Zahnextraktionen* & & $3,1 \pm 4,1$ \\
\hline 0 & $15(28,8)$ & \\
\hline $1-9$ & $33(63,5)$ & \\
\hline $10-19$ & $4(7,7)$ & \\
\hline$\geq 20$ & 0 & \\
\hline Zahnentfernung aufgrund kariöser Destruktion & & $2,5 \pm 3,8$ \\
\hline Zahnentfernung aufgrund parodontaler Destruktion & & $0,9 \pm 2,1$ \\
\hline Zahnfüllungen* & & $4,7 \pm 4,4$ \\
\hline 0 & $9(17,3)$ & \\
\hline $1-9$ & $35(67,3)$ & \\
\hline $10-19$ & $7(13,4)$ & \\
\hline$\geq 20$ & $1(1,9)$ & \\
\hline Parodontalbehandlung & & \\
\hline Keine & $1(1,9)$ & \\
\hline Professionelle Zahnreinigung & $4(7,7)$ & \\
\hline Professionelle Zahnreinigung und Deep Scaling & $47(90,0)$ & \\
\hline Wurzelkanalbehandlung & $1(1,9)$ & \\
\hline
\end{tabular}

\subsection{Signifikante Einflussfaktoren auf die MLQ vor der Therapie}

Die Merkmale der Anamnese und die Parameter der Mundgesundheit wurden als potenzielle Einflussfaktoren (Prädiktoren) auf die MLQ (OHIP-G14- und OHIP-Ge-Summenwert) zum Zeitpunkt T0 (Baseline) überprüft. Aufgrund mangelnder Ausprägung (kein Patient war parodontal gesund und nur ein Patient erhielt eine Wurzelkanalbehandlung) konnten die Variablen „parodontale Verdachtsdiagnose“ und „Wurzelkanalbehandlung“ nicht berücksichtigt werden. In 
Tabelle 4-9 sind alle signifikanten Ergebnisse der mehrfaktoriellen Regressionsanalyse dargestellt.

Es konnte ein signifikanter Zusammenhang zwischen einem parodontalen Behandlungsbedarf im 6. Sextanten (odds ratio: 1,$81 ; p=0,044$ ) und dem OHIPG14-Summenwert festgestellt werden. Patienten mit einem parodontalen Behandlungsbedarf im 6. Sextanten hatten ein um 80 \% erhöhtes Risiko für eine reduzierte MLQ. Außerdem konnte die Ätiologie der kognitiven Einschränkung als signifikanter Prädiktor des OHIP-G14-Summenwertes bestätigt werden. Die Betreuer bewerteten die MLQ von Patienten mit erworbener kognitiver Einschränkung (odds ratio: 0,12; $p<0,001$ ) als weniger stark eingeschränkt im Vergleich zu Patienten, bei denen die kognitive Einschränkung seit der Geburt bestand. Ebenso bewerteten Betreuer von Patienten mit einer progredienten kognitiven Einschränkung deren MLQ als weniger stark eingeschränkt (odds ratio: 0,$11 ; p=0,002)$. Für alle anderen Variablen wie Alter, Geschlecht oder Zahnanzahl konnte kein signifikanter Einfluss auf den OHIP-Summenwert zum Zeitpunkt TO festgestellt werden.

Für den OHIP-Ge konnte kein signifikanter Einflussfaktor auf die MLQ zum Zeitpunkt T0 ermittelt werden.

Tabelle 4-9: Signifikante Einflussfaktoren auf den OHIP-G14 zum Zeitpunkt T0.

\begin{tabular}{|l|c|c|c|}
\hline \multicolumn{1}{|c|}{ Einflussfaktoren zu T0 } & $\begin{array}{c}\text { odds } \\
\text { ratio }\end{array}$ & Konfidenzintervall & p-Wert \\
\hline Erworbene kognitive Einschränkung & 0,12 & {$[0,029 ; 0,424]$} & 0,002 \\
\hline Progrediente kognitive Einschränkung & 0,11 & {$[0,028 ; 0,454]$} & 0,002 \\
\hline PSI 6.Sextant & 1,81 & {$[1,023 ; 3,283]$} & 0,044 \\
\hline
\end{tabular}

\subsection{Signifikante Einflussfaktoren auf die MLQ im Verlauf der Studie}

Die signifikanten Einflussfaktoren auf die Veränderung des OHIP-G14Summenwerts sind in Tabelle 4-10 dargestellt.

Bei Patienten mit progredienter kognitiver Einschränkung reduzierte sich der OHIP-G14-Summenwert zwischen dem Baselinezeitpunkt (T0) und dem Zeitpunkt T1 weniger stark als bei Patienten mit erworbener oder angeborener kog- 
nitiven Einschränkung (odds ratio: 18,23; $\mathrm{p}<0,001$ ). Die Reduktion der OHIPG14-Summenwerte war im Studienverlauf (T0 bis T2) bei Patienten mit erworbener kognitiver Einschränkung (odds ratio: 6,00; $p=0,006$ ) und besonders bei Patienten mit progredienter kognitiver Einschränkung (odds ratio: 14,92; $\mathrm{p}<0,001)$ geringer als bei Patienten mit angeborener kognitiver Einschränkung. Je mehr Weisheitszähne in der ITN gefüllt wurden, desto weniger reduzierte sich der OHIP-Summenwert von T0 zu T1. Bei Betreuungspersonen, die mehr Zeit pro Tag mit dem Patienten verbrachten, reduzierte sich der OHIP. Summenwert von T1 zu T2 stärker (odds ratio: 0,87; $p=0,028$ ), als bei Betreuungspersonen, die weniger Stunden pro Tag mit dem Patienten verbrachten.

Tabelle 4-10: Signifikante Einflussfaktoren auf die Summenwertänderung des OHIP-G14. Je stärker die Odds ratio von 1 abweicht (sowohl OR $>1$ als auch $O R<1$ ), desto stärker ist die Veränderung des Summenswertes über die Zeit. Ein $O R<1$ bedeutet eine Verbesserung des Summenwertes über die Zeit.

\begin{tabular}{|c|c|c|c|}
\hline \multicolumn{1}{|c|}{$\begin{array}{c}\text { Einflussfaktoren } \\
\text { Veränderung T0 zu T1 }\end{array}$} & $\begin{array}{c}\text { odds } \\
\text { ratio }\end{array}$ & Konfidenzintervall & $\mathbf{p}$-Wert \\
\hline Progrediente kognitive Einschränkung & 18,23 & {$[3,572 ; 112,011]$} & $<0,001$ \\
\hline $\begin{array}{c}\text { Anzahl an Füllungen } \\
\text { (inklusive Weisheitszähne) }\end{array}$ & 5,53 & {$[1,185 ; 27,682]$} & 0,031 \\
\hline $\begin{array}{c}\text { Einflussfaktor } \\
\text { Veränderung T1 zu T2 }\end{array}$ & $\begin{array}{c}\text { odds } \\
\text { ratio }\end{array}$ & Konfidenzintervall & $\mathbf{p}$-Wert \\
\hline Betreuungszeit pro Tag & 0,87 & {$[0,762 ; 0,983]$} & 0,028 \\
\hline $\begin{array}{c}\text { Einflussfaktoren } \\
\text { Veränderung T0 zu T2 }\end{array}$ & $\begin{array}{c}\text { odds } \\
\text { ratio }\end{array}$ & Konfidenzintervall & $\mathbf{p - W e r t}$ \\
\hline Erworbene kognitive Einschränkung & 6,00 & {$[1,722 ; 22,354]$} & 0,006 \\
\hline Progrediente kognitive Einschränkung & 14,92 & {$[3,646 ; 66,847]$} & $<0,001$ \\
\hline Erhöhter PSI im 6. Sextanten & 0,55 & {$[0,316 ; 0,928]$} & 0,026 \\
\hline
\end{tabular}

Tabelle 4-11 zeigt die signifikanten Einflussfaktoren auf die OHIP-GeSummenwertänderung zwischen den einzelnen Befragungszeitpunkten. Je mehr Zähne in ITN entfernt wurden, desto weniger reduzierte sich der OHIPGe-Summenwert von T0 zu T2 (odds ratio: 1,23; $p=0,025$ ). Je mehr Zähne mit Füllungen versorgt wurden, desto eher verbesserte sich die MLQ (odds ratio: $0,84 ; p=0,028)$. 
Tabelle 4-11: Signifikante Einflussfaktoren auf die Summenwertänderung des OHIP-Ge. Je stärker die odds ratio von 1 abweicht (sowohl $O R>1$ als auch $O R<1$ ), desto stärker ist die Veränderung des Summenswertes über die Zeit. Ein $\mathrm{OR}<1$ bedeutet eine Verbesserung des Summenwertes über die Zeit.

\begin{tabular}{|c|c|c|c|}
\hline $\begin{array}{c}\text { Einflussfaktor } \\
\text { Veränderung T0 zu T1 }\end{array}$ & $\begin{array}{c}\text { odds } \\
\text { ratio }\end{array}$ & Konfidenzintervall & $\mathbf{p}$-Wert \\
\hline Anzahl an Füllungen & 0,84 & {$[0,713 ; 0,978]$} & 0,028 \\
\hline $\begin{array}{c}\text { Einflussfaktoren } \\
\text { Veränderung T1 zu T2 }\end{array}$ & $\begin{array}{c}\text { odds } \\
\text { ratio }\end{array}$ & Konfidenzintervall & $\mathbf{p}$-Wert \\
\hline Anzahl Extraktionen aufgrund kariöser Destruktion & 1,49 & {$[1,16 ; 2,06]$} & 0,007 \\
\hline Anzahl suffizient gefüllter Zähne nach der Therapie & 0,76 & {$[0,61 ; 0,94]$} & 0,012 \\
\hline $\begin{array}{c}\text { Einflussfaktor } \\
\text { Veränderung T0 zu T2 }\end{array}$ & $\begin{array}{c}\text { odds } \\
\text { ratio }\end{array}$ & Konfidenzintervall & $\mathbf{p}$-Wert \\
\hline $\begin{array}{c}\text { Anzahl an Zahnentfernungen } \\
\text { (ohne Weisheitszähne) }\end{array}$ & 1,23 & {$[1,026 ; 1,481]$} & 0,025 \\
\hline
\end{tabular}




\section{Diskussion}

Ziel dieser Studie war es zu untersuchen, ob und wie sich die zahnärztliche Behandlung in ITN auf die MLQ von Patienten mit kognitiver Einschränkung auswirkt. Bislang wurden Auswirkungen dieser komplexen zahnmedizinischen Behandlung nur in einer koreanischen Studie und ausschließlich über die Fremdbefragung der Betreuungspersonen untersucht (Chang et al. 2014). In der vorliegenden Studie wurden erstmals zusätzlich zur Fremdbefragung der Betreuungspersonen auch die Patienten selbst zu ihrer MLQ befragt. So konnte die Korrelation von Patienten- und Betreuerbefragung untersucht werden. Neben klinischen Parametern (vor und nach der zahnmedizinischen Behandung) und den im Rahmen der ITN erfolgten Therapiemaßnahmen wurden behinderungsassoziierte Faktoren auf die Bewertung der MLQ durch die Betreuungspersonen (Fremdbefragung) und auf die Bewertung der MLQ durch die Patienten (Selbstbefragung) überprüft.

\subsection{Patientenkollektiv}

Alle Daten der vorliegenden Untersuchung stammen von Patienten der Poliklinik für Präventive Zahnmedizin Parodontologie und Kariologie der Universitätsmedizin Göttingen. Die Stichprobe zeichnet sich durch eine große Bereitschaft zur Studienteilnahme (79,2\% der befragten Betreuer willigten in die Studienteilnahme ein), eine moderate Dropout-Rate (bei $11,5 \%$ der Patienten fand die geplante Therapie in ITN nicht statt) und eine sehr geringe Lost-to-followup-Rate (zwei Patienten nahmen die Termine zur Befragung nach der ITN nicht wahr) aus.

Die vorliegende Studie erfasst in Bezug auf Ursache, Art und Schwere der kognitiven Einschränkung eine heterogene Personengruppe. Die Diversität der Ausprägung einer kognitiven Einschränkung ist typisch für die Personengruppe mit kognitiver Einschränkung und erschwert einen homogenen und objektiven Einschluss dieser Patienten in Forschungsarbeiten. Zur Vermeidung von Diskriminierungen und Fehleinschätzungen sollte die Definition der Behinderung bei Forschungsarbeiten im fachspezifischen Kontext erfolgen (Carroll 1993; 
Pörtner 2006; Janz und Terfloth 2009). Die fachspezifische Definition der kognitiven Einschränkung erfolgte in der vorliegenden Studie über die zahnmedizinische funktionelle Kapazität der Patienten. Alle Patienten entsprachen aufgrund ihrer kognitiven Einschränkung der Belastungsstufe 4 nach Nitschke und Hopfenmüller (1991). Aus der kognitiven Einschränkung resultierte bei allen Patienten eine so stark reduzierte Kooperationsfähigkeit, dass eine zahnmedizinische Therapie im Wachzustand nicht möglich war. Bei allen Patienten lag folglich eine zahnmedizinisch hochrelevante kognitive Einschränkung vor. Das Konzept der zahnmedizinischen funktionellen Kapazität erfültt als Kriterium die Ansprüche an eine fachrelevante Definition der Behinderung. Zum objektiven Nachweis des Bestehens einer kognitiven Einschränkung wurde das Vorliegen eines gesetzlichen Betreuungsverhältnisses als Einschlusskriterium gewählt. Auch Schulte et al. (2013) beziehen sich in ihrer Studie zur Analyse des Mundgesundheitszustandes von Personen mit kognitiver Einschränkung auf Personen, die sich in einem gesetzlichen Betreuungsverhältnis befinden. Andere zahnmedizinische Studien wählen als Einschlusskriterium spezielle Diagnosen, die als ursächlich für die kognitive Einschränkung angesehen werden (Cheng et al. 2007; Jaber 2011; Frydman und Nowzari 2012; Rodríguez Peinado et al. 2018). Aufgrund der großen Bandbreite an Auswirkungen und Ausprägungen einer Diagnose, ist die Diagnose in Bezug auf die zahnmedizinische Therapiefähigkeit wenig aussagekräftig. Die Diagnosen in Bezug auf die Ursache der kognitiven Einschränkung der Patienten waren in der vorliegenden Studie sehr unterschiedlich. Daher wurde auf eine Zuordnung hinsichtlich der Diagnose verzichtet. Die Verteilung und Ausprägung behinderungsassoziierter Merkmale war, wie erwartet, divers. Bei dem Großteil der Patienten bestand zusätzlich eine körperliche Einschränkung. Zur Kompensation behinderungsassoziierter Defizite wurden, bis auf eine Person, alle Studienteilnehmer im Alltag von einer Betreuungsperson begleitet. Die Betreuungszeit pro Tag war unterschiedlich hoch, was vermutlich einen unterschiedlichen Unterstützungsbedarf der Patienten widerspiegelt. In Anbetracht der Schwere der Einschränkungen (keine selbstständige Bewältigung des Alltags, teilweise keine Kommunikatonsfähigkeit, teilweise keine eigenständige Nahrungsaufnahme) ist von einer stark bis sehr stark eingeschränkten und größtenteils mehrfachbehinderten Patientenklientel auszugehen. 
Fast alle Patienten der vorliegenden Studie wiesen einen schlechten parodontalen Gesundheitszustand auf. Nach übereinstimmender Auffassung der Autoren, welche die Mundgesundheit von Personen mit kognitiver Einschränkung erfassen, muss das erhöhte Vorkommen von Parodontopathien bei dieser Personengruppe im Zusammenhang mit der meist inadäquaten Mundhygiene betrachtet werden (Tiller et al. 2001; Pezzementi und Fisher 2005; Anders und Davis 2010; Leroy und Declerck 2013; Kothari et al. 2017). Auch nationale Untersuchungen zeigen, dass bei der Mehrheit der Personen mit kognitiver Einschränkung eine Gingivitis bei inadäquater Mundhygiene besteht. Eine ausführliche parodontale Diagnostik erfolgte allerding nicht (Schulte et al. 2011; Schulte et al. 2013). Personen mit kognitiver Einschränkung sind zum Gesunderhalt der oralen Strukturen häufig auf Unterstützung bei der Mundhygiene angewiesen. Nach den Angaben der Betreuungspersonen erhielten 27 (52,9\%) Studienpatienten Hilfe bei der Durchführung der täglichen Zahnpflege; bei 9 (17,6\%) Patienten gaben die Betreuungspersonen an, dass die Durchführung von häuslichen Mundhygienemaßnahmen nicht möglich sei. Der zahnmedizinische Behandlungsbedarf der Studienpatienten (vor der Therapie in ITN) zeigt, dass die Mundhygienemaßnahmen bei keinem der Patienten zum Gesunderhalt der oralen Strukturen ausreichten. Die Patienten der vorliegenden Studie hatten einen ähnlich hohen parodontalen Behandlungsbedarf, wie die von Pieper et al. (1986) vor über 30 Jahren ebenfalls in Göttingen untersuchten Personen mit kognitiver Einschränkung. Pieper et al. (1986) stellten allerdings eine wesentlich höhere Kariesprävalenz bei den Personen mit kognitiver Einschränkung fest. Der DMF-T betrug damals in der Gruppe der 17- bis 24-Jährigen 17,4 und in der Gruppe der 55- bis 64-jährigen Probanden 26,9. Der DMFT-T betrug bei der Klientel der vorliegenden Studie durchschnittlich 13,7 $\pm 7,3$. Durchchschnittlich waren pro Patient 6,7 \pm 5,9 Zähne kariös. Aktuellere epidemiologische Studien deuten daraufhin, dass sich die Kariesprävalenz bei Personen mit kognitiver Einschränkung innerhalb der letzten Jahrzehnte in Deutschland deutlich reduziert hat (Schulte et al. 2011; Schulte et al. 2013, Tabelle 5-1). Es handelt sich bei diesen Studien jedoch um die Ergebnisse zahnmedizinischer Untersuchungen von kooperativen, leicht bis mittelschwer kognitiv beeinträchtigten Personen, die nicht beschwerdeorientiert untersucht wurden. Im Vergleich mit den Studien von Schulte et al. $(2011 ; 2013)$ waren die DMF-T-Werte vergleichbar, 
allerdings wiesen die Patienten der vorliegenden Studie einen höheren Anteil unbehandelter kariöser Zähne (D-T-Wert) auf, wahrscheinlich weil sie beschwerdeorientiert in der Klinik vorgestelt wurden.

Eine Erklärung für die Verringerung der allgemeinen Kariesprävalenz, bei unveränderter Parodontitisprävalenz, ist der Einsatz von mundhygieneunabhängigeren Präventionsmaßnahmen (Ernährungslenkung, Fluoridierung). Eine gering kariogene Ernährung und Flouridierungsmaßnahmen wirken kariesprotektiv, schützen aber nicht vor parodontalen Erkrankungen (Hellwege 2003). Die Anzahl an kariösen und fehlenden Zähnen vor der Therapie war innerhalb der Patientenklientel unterschiedlich hoch und ist als Ergebnis individueller protektiver und pathogener Einflussfaktoren auf die Zahngesundheit zu interpretieren. Die zahnmedizinische Therapie in ITN verbesserte die konservierende Versorgungssituation der Studienpatienten auf ein mit der Allgemeinbevölkerung vergleichbares Niveau (Tabelle 5-1). Die verbesserte Versorgungssituation entspricht der UN-Behindertenrechtskonvention (2009), die spezielle medizinische Maßnahmen zur Kompensation behinderungsassoziierter Gesundheitseinschränkungen fordert. Vergleichbare nationale Daten zum Mundgesundheitszustand von Patienten, die aufgrund von unkooperativem Verhalten nur im Rahmen einer Narkose zahnmedizinisch behandelt werden können, liegen aktuell nicht vor. 
Tabelle 5-1: Vergleich der Ergebnisse zur Karieserfahrung mit anderen nationalen kariesepidemiologischen Studien

\begin{tabular}{|c|c|c|c|c|c|}
\hline & $\begin{array}{c}\text { Fünfte Deutsche } \\
\text { Mundgesundheitsstu- } \\
\text { die }\end{array}$ & Special Olympics & $\begin{array}{l}\text { Betreuungs- } \\
\text { einrichtungen }\end{array}$ & \multicolumn{2}{|c|}{ Vorliegende Studie } \\
\hline & Jordan et al. 2016 & Schulte et al. 2011 & Schulte et al. 2013 & $\begin{array}{l}\text { vor der } \\
\text { Therapie }\end{array}$ & $\begin{array}{l}\text { nach der } \\
\text { Therapie }\end{array}$ \\
\hline $\begin{array}{l}\text { Studien- } \\
\text { klientel }\end{array}$ & $\begin{array}{l}\text { Allgemeinbevölkerung } \\
\text { (Alter: } 35 \text { bis } 44 \text { Jahre) }\end{array}$ & $\begin{array}{l}\text { Kooperative Personen } \\
\text { mit kognitiver } \\
\text { Einschränkung } \\
\text { (Alter: } 35 \text { bis } 44 \text { Jahre) }\end{array}$ & $\begin{array}{l}\text { Kooperative Personen } \\
\text { mit kognitiver } \\
\text { Einschränkung } \\
\text { (Alter: } 35 \text { bis } 44 \text { Jahre) }\end{array}$ & \multicolumn{2}{|c|}{$\begin{array}{l}\text { Unkooperative Personen mit } \\
\text { kognitiver Einschränkung } \\
\text { (Alter: } 38 \pm 13,5 \text { Jahre) }\end{array}$} \\
\hline $\begin{array}{l}\text { Unter- } \\
\text { suchungs- } \\
\text { zeitraum }\end{array}$ & 2014 & 2008 & 2007/08 & \multicolumn{2}{|c|}{ 2015-17 } \\
\hline DMF-T & 11,2 & 13,5 & 12,3 & \multicolumn{2}{|c|}{13,7} \\
\hline D-T & 0,5 & 0,9 & 1,8 & 6,7 & 0 \\
\hline M-T & 2,1 & 4,2 & 6,1 & 3,3 & 5,6 \\
\hline F-T & 8,6 & 8,4 & 5,7 & 3,7 & 8,1 \\
\hline
\end{tabular}

Der schlechte gingivale/parodontale Gesundheitszustand und der durchschnittliche DMF-T-Wert der Studienpatienten entsprechen (unter Berücksichtigung des speziellen Settings) der aktuelleren nationalen und internationalen Studienlage zum Mundgesundheitszustand von Personen mit kognitiver Einschränkung (Kaschke 2008; Anders und Davis 2010; Schulte et al. 2011; Frydman und Nowzari 2012; Schulte et al. 2013; Scalioni et al. 2018).

\subsection{Studiendesign}

Mundgesundheit ist ein wichtiger Einflussfaktor der Allgemeingesundheit und der MLQ. In der vorliegenden Studie wurde, wenn es die Kommunikationsfähigkeit der Patienten erlaubte, die MLQ auch über die Selbstbefragung der Patienten quantifiziert. Die Selbstbefragung stellt den Goldstandard zur Bestimmung der MLQ dar (Locker und Slade 1994; John et al. 2016). Bei Patienten, die sel- 
ber nicht direkt kommunizieren können oder wollen, ist die Fremdbefragung das Mittel der Wahl (Zhu et al. 2008; McGrath et al. 2009).

Kommunikationsdefizite erschweren den Einsatz von quantitativen Methoden zur Erforschung von Fragestellungen in Bezug auf die Lebensqualität bei Personen mit kognitiver Einschränkung (Janz 2009). Eine Fremdbefragung sollte die subjektive Einschätzung des Patienten widerspiegeln. Vergleiche von Selbstbefragungen bei Kindern und Fremdbefragungen von deren Eltern haben gezeigt, dass Fremdbefragungen und Selbstbefragungen zwar korrelieren, es aber (besonders bei älteren Kindern) zu Fehlinterprationen der MLQ durch die Eltern und damit zu Verzerrungen der Ergbnisse kommt. Bei der Quantifikation der kindlichen MLQ wird daher zusätzlich zur Fremdbefragung eine an das Alter angepasste Befragung der Kinder selbst empfohlen (Eiser und Morse 2001; Wilson-Genderson et al. 2007; Lee et al. 2017; Reissmann et al. 2017). Da zu Studienbeginn keine Fragebogeneinstrumente zur Selbstbefragung von erwachsenen Personen mit (schwerer) kognitiver Einschränkung existierten, wurde die validierte Ultrakurzversion des OHIPs mit nur fünf Fragen (OHIP-G5) an die reduzierten kognitiven Fähigkeiten angepasst. Die Fragen wurden in Leichte Sprache überführt und vom Netzwerk für Leichte Sprache ${ }^{2}$ als einfach zu verstehendes Dokument zertifiziert (OHIP-Ge). Durch Anpassung der Ultrakurzversion sollte der reduzierten Aufmerksamkeitsfähigkeit der Patienten entsprochen werden. Die Patienten, die auf den OHIP-Ge antworteten, beantworteten stets alle fünf Fragen, was für eine angemessene Länge des Fragebogens spricht. Die Befragungen der Patienten fanden räumlich getrennt von ihren Betreuungspersonen statt, um eine gegenseitige Beeinflussung ausschließen zu können. Die psychometrischen Eigenschaften des verwendeten OHIP-G14 und des OHIP-G5 gelten als gut und valide (John et al. 2004). Als Limitation dieser Studie muss jedoch festgehalten werden, dass der für diese Studie modifizierte OHIP-G-Fragebogen nicht für den Einsatz bei Personen mit kognitiver Einschränkung validiert ist. Eine Validierung des OHIP-Ge war im Rahmen dieser Studie aufgrund der geringen Patientenzahl $(n=27)$ und der fehlenden Kontrollgruppe nicht möglich. Auf die Überprüfung der Retest-Reliabilität wurde ver-

\footnotetext{
${ }^{2}$ Büro für Leichte Sprache und Barrierefreiheit, Süntelstraße 32a, 49088 Osnabrück
} 
zichtet, um dem oft schwerstmehrfachbehinderten Patientenklientel den studienbedingten Mehraufwand zu ersparen. Das Studiendesign entspricht den Anforderungen zur personenzentrierten Forschung mit Menschen mit Behinderung (fachspezifische Definition der kognitiven Einschränkung, aktive Integration der Personen mit kognitiver Einschränkung in den Forschungsprozeß) und zeigt, trotz der Limitationen, eine Möglichkeit zur quantitativen Bestimmung der $M L Q$ von Personen mit kognitiver Einschränkung auf.

Die Auswertung der OHIP-Fragebögen erfolgte entsprechend der Empfehlung von John et al. (2014) durch die Analyse der Summenwerte von Anfangs- und Folgebefragungen. Eine weitere Möglichkeit der Auswertung ist die Analyse einzelner Items oder die Zuordnung der Items zu einzelnen Dimensionen der MLQ. Diese Arten der Auswertung erlauben eine differenziertere Analyse der Einschränkungen der MLQ als die Darstellung des Summenwertes. Da die Zuordnung der Items zu den einzelnen Dimensionen und auch die Anzahl der Dimensionen in der Literatur nicht einheitlich empfohlen werden, wurde in dieser Studie auf diese Art der Auswertung verzichtet (John et al. 2004; John 2007). Die durchgeführte Auswertung über die Summenwerte ermöglicht einen Vergleich der vorliegenden Studienergebnisse mit den Ergebnissen anderer Studien und mit den Normwerten der Allgemeinbevölkerung (John 2004).

Es sollte primär überpüft werden, ob sich die MLQ der beschriebenen Patientenklientel durch eine Zahnsanierung in ITN verändert. Die Zahnsanierung zielt auf die Wiederherstellung der Mundgesundheit ab. Mundgesundheit gilt als wichtiger Einflussfaktor der MLQ. Zur Beschreibung der vielschichtigen Mundgesundheit (vor und nach der der Therapie) sowie zur Quantifikation der MLQ wurden etablierte Indizes verwendet. Dieses Vorgehen ermöglicht einen (internationalen) Vergleich des Mundgesundeheitszustandes und der MLQ der untersuchten Patienten. Da die Daten zum Mundgesundheitszustand der vorliegenden Studie in ITN erhoben wurden, konnten alle Patienten, auch solche, die sich im Wachzustand bei der zahnmedizinischen Diagnostik nicht ausreichend kooperativ zeigten, berücksichtigt werden. Die Daten zur Mundgesundheit, wie auch alle weiteren potenziellen Einflussfaktoren (Therapiemaßnahmen in ITN, anamnestische Faktoren) auf die MLQ, wurden den Patientenakten entnommen. So beschränkte sich der studieninitiierte Mehraufwand für die Studienteil- 
nehmer auf das Beantworten der MLQ-Fragebögen zu den Untersuchungsbzw. Kontrollterminen. Chang et al. (2014) stellten fest, dass es aufgrund der Komplexität und Variabilität der zahnmedizinischen Behandlungsmaßnahmen in ITN schwierig sei, zu beurteilen, welche einzelnen Behandlungsmaßnahmen ursächlich für die Veränderung der MLQ nach der Therapie sind (Chang et al. 2014). Auch im Rahmen dieser Studie überlagerten sich die potenziellen Einflussfaktoren, zusätzlich waren die Ausprägungen der potenziellen Einflussfaktoren divers. Trotz der Vielzahl an Variablen und der relativ kleinen Patientenklientel konnten im Rahmen dieser Studie einzelne signifikante Einflussfaktoren auf die MLQ vor der Therapie und auch auf die Veränderung der MLQ im Verlauf der Therapie verifiziert werden. Mit einer höheren Anzahl an Studienteilnehmern (multizentrisches Studiendesign oder längere Studienlaufzeit) könnten zukünftig eventuell weitere signifikante Einflussfaktoren auf die MLQ der Patienten mit kognitiver Einschränkung verifiziert werden.

Die Befragung vor und zu zwei Terminen nach der Behandlung in Vollnarkose ermöglicht eine detaillierte Darstellung der Veränderung der MLQ. Ähnliche Befragungsabstände wurden zur Evaluation der MLQ im Verlauf von Parodontitistherapien gewählt (Mendez et al. 2017). Therapien, die den Einfluss von prothetischen Versorgungen auf die MLQ evaluieren, wählen teilweise deutlich längere Befrangungsabstände bei längeren Studienlaufzeiten (Aarabi et al. 2015; Fischer et al. 2015). Chang et al. (2014) befragen die Betreuungspersonen vier Wochen nach Therapie in ITN. Ein fester Bezugszeitraum wird generell bei der Verwendung des OHIPs empfohlen (Slade und Spencer 1994). Waller et al. (2016) stellen fest, dass ein Abstand von sieben Tagen zwischen zahnmedizinischer Intervention und Befragung ausreicht, um verlässliche Aussagen über eine Veränderung der MLQ zu erhalten. Eine Befragung zu wesentlich späteren Zeitpunkten birgt die Gefahr von Verzerrungen durch Ereignisse, die in keinem direkten Zusammenhang mit der zahnmedizinischen Intervention stehen. Besonders bei der im Rahmen dieser Studie evaluierten unkooperativen Patientengruppe gestaltet es sich schwierig, den im Rahmen der ITN hergestellten Mundgesundheitszustand durch präventive Maßnahmen langfristig aufrechtzuerhalten. Die Aussagekraft der Studie ist durch den Zeitpunkt der letzten Befragung auf drei Monate nach der Therapie limitiert. Es wäre denkbar, dass sich 
die MLQ nach den drei Monaten weiter verändert. In diesem Zusammenhang wäre es interessant, in zukünftigen Studien zu untersuchen, wie lange nach einer entsprechenden zahnmedizinischen Behandlung in Vollnarkose ein (positiver) Effekt auf die MLQ nachweisbar ist.

\subsection{Ergebnisse}

Vor der Therapie lagen die Einschränkungen der MLQ hauptsächlich in dem Bereich "Schmerz". Nach der Therapie verbesserte sich die Bewertung der MLQ sowohl in der Fremd- als auch in der Selbstbefragung signifikant. Drei Monate nach der Therapie lagen nur noch vereinzelte Einschränkungen der MLQ vor; diese beschränkten sich auf funktionelle und psyochosoziale Bereiche.

\subsubsection{Bewertung der MLQ durch die Fremdbefragung}

Die Ergebnisse der Fremdbefragung sprechen für eine Verbesserung der MLQ durch die orale Rehabilitation in ITN. Alle Patienten wurden von den Betreuungspersonen beschwerdeorientiert, mit dem Wunsch nach einer zahnmedizinischen Behandlung, vorgestellt. Gründe für das Vorstellen in der Klinik zum Zeitpunkt T0 waren bei 35,3\% der Patienten Schmerzen, bei 15,7\% der Patienten wurden Schwellungen im Gesicht bemerkt und bei 21,6 \% der Patienten befürchtete die Betreuungsperson, dass Karies bestehen könnte. Es war also davon auszugehen, dass die Betreuungspersonen die MLQ der Patienten als eingeschränkt einschätzten. Die von den Betreuungspersonen vermuteten Beschwerden spiegeln sich in ihrer Fremdeinschätzung in den erhöhten OHIPG14-Summenwerten vor der Therapie wider. Die Einschränkungen der MLQ resultierten dabei hauptsächlich aus Einschränkungen in dem Bereich „Schmerzen“. Die Betreuer gaben seltener Einschränkungen in den Bereichen Ästhetik, Funktion oder soziale Akzeptanz an. Ein Grund hierfür könnte sein, dass die Patienten aufgrund der Ausprägung ihrer Behinderung generell in den Dimensionen Funktion, Ästhetik und soziale Interaktion beeinträchtigt sind und der eingeschränkte Zustand von den Betreuern als unveränderbarer Status akzeptiert wird. 
Die Betreuer bewerteten die MLQ der Patienten vor der Therapie (bei zahnmedizinischem Behandlungsbedarf) mit einem OHIP-G14-Summenwert von 18,8 \pm 11,6. Der Vergleich der OHIP-Summenwerte mit den Normwerten der deutschen Allgemeinbevölkerung (Personen ohne Zahnersatz: OHIP-G14Summenwert 0; Personen mit herausnehmbaren Zahnersatz: OHIP-G14Summenwert 4, Personen mit Totalprothesen: OHIP-G14-Summenwert 6) bestätigt eine stark eingeschränkte MLQ der Patienten vor der zahnmedizinischen Behandlung (Meyer et al. 2004).

Die Bewertung der MLQ verbesserte sich nach der zahnmedizinischen Therapie signifikant. Auch Chang et al. (2014) konnten durch eine Fremdbefragung der Betreuungspersonen eine Verbeserung der MLQ von erwachsenen Patienten mit kognitiver Einschränkung nach zahnmedizinischer Behandlung in ITN feststellen. Entsprechend den Ergebnissen von Chang et al. (2014) konnte ausserdem die Ätiologie der kognitiven Einschränkung als signifikanter Prädiktor des OHIP-Summenwertes bei der Fremdbefragung verifiziert werden. Die Betreuer bewerteten die MLQ von Patienten mit erworbener kognitiver Einschränkung als weniger eingeschränkt im Vergleich zu Betreuern von Patienten, bei denen die kognitive Einschränkung seit der Geburt bestand. Naheliegend wäre, dass sich bei längerem Bestehen der kognitiven Einschränkung, also bei Personen mit angeborener Behinderung, die aus der kognitiven Einschränkung resultierenden Lebensbedingungen über den langen Zeitraum besonders negativ auf die Mundgesundheit und damit auch negativ auf die MLQ auswirken. Eventuell bestehen bei Personen mit erworbener kognitiver Einschränkung und bei Personen mit neurodegenrativen Erkrankungen weitere, ebenfalls akute somatische Einschränkungen, welche die Einschränkungen der MLQ überlagern. Wenn neben den oralen Erkrankungen weitere gesundheitliche Einschränkungen bestehen, werden die oralen Beschwerden eventuell als weniger wichtig erachtet. Der Zusammenhang von gesundheitsbezogener und mundgesundheitsbezogener Lebensqualität gilt als sicher (Sischo und Broder 2011). Eine weitere Erklärung wäre, dass Personen, die von Geburt an kognitiv eingeschränkt sind, an die reduzierten allgemeinen Lebensbedingungen adaptiert sind und die Betreuer deshalb die speziellen Einschränkungen der MLQ kritischer bewerten. Eine Person, die nach einem Unfall plötzlich nicht mehr selbst- 
ständig laufen kann, wird sich vermutlich weniger über einen abgebrochenen Zahn ärgern. Kann eine Person aber seit der Geburt nicht laufen, ist dieser Zustand Altag und andere Einschränkungen, wie ein abgebrochener Zahn, gewinnen an Bedutung.

Im Rahmen der vorliegenden Studie konnte festgestellt werden, dass Personen mit einem parodontalen Behandlungsbedarf im 6. Sextanten einen signifikant erhöhten OHIP-G14-aufwiesen. Es konnte allerdings kein klarer Zusammenhang zwischen einem generellen parodontalen Behandlungsbedarf (erhöhter PSI in allen Sextanten) und einer reduzierten MLQ verifiziert werden. Da fast alle Patienten als Verdachtsdiagnose eine Parodontitis aufwiesen, konnte keine vergleichende Analyse mit Patienten ohne diese Verdachtsdiagnose durchgeführt werden. Andere Studien belegen einen Zusammenhang von parodontaler Erkrankung und reduzierter MLQ bei Personen ohne kognitive Einschränkung (Ferreira et al. 2017). Ebenso konnte auch bei Personen mit Down Syndrom ein negativer Einfluss einer parodontalen Erkrankung auf die MLQ festgestellt werden (Loureiro et al. 2007). In der vorliegenden Studie war bei dem Bestehen einer parodontalen Schädigung (im 6. Sextanten) der OHIP-G14 zum Baselinezeitpunkt erhöht und reduzierte sich nach der Therapie in ITN signifikant stärker. Jowett et al. (2009) zeigten ebenfalls, dass sich nach einem Deep Scaling (bei Patienten ohne kognitive Einschränkung) der Medianwert des OHIP 14 von einem Summenwert von 5 auf einen Summenwert von 2 (nach einer Woche) verringerte. In der vorliegenden Studie bestanden drei Monate nach der Therapie keine wesentlichen Einschränkungen der MLQ mehr. Im Rahmen der ITN erfolgten neben dem Deep Scaling weitere therapeutische Maßnahmen, wobei die Füllungstherapie als signifikanter Einflussfaktor auf die Reduktion des OHIP-G14-Summenwertes zwischen T0 und T1 verifiziert werden konnte. Je mehr Zähne nach der Therapie gefüllt waren, desto eher reduzierte sich der OHIP-G14-Summenwert zwischen den Zeitpunkten T0 und T1. Dies ist eventuell mit der durch die Füllungstherapie beseitigten Schmerzen zu erklären. Wenn im Rahmen der ITN Zähne entfernt wurden, beeinflusste dies die Summenwertreduktion eher negativ und zwar sowohl von T0 zu T2, wie auch von T1 zu T2. Trotzdem verbesserte sich die MLQ auch bei Patienten, bei denen Zähne entfernt wurden, wenn auch weniger stark. Es ist denkbar, dass sich der Zahnver- 
lust bzw. der Verlust der vertikalen Abstützung erst nach längerer Zeit negativ auf die MLQ auswirkt. Die Reduktion der OHIP-G14-Summenwerte war im Studienverlauf (T0 bis T2) bei Patienten mit erworbener kognitiver Einschränkung und bei Patienten mit progredienter kognitiver Einschränkung geringer als bei Patienten mit angeborener kognitiver Einschränkung. Für weitere Variablen konnte - eventuelll auch aufgrund der heterogenen Ausprägungen und der damit verbundenen geringen Fallzahl - kein signifikanter Einfluss auf den OHIPG14-Summenwert festgestellt werden. Einen Zusammenhang zwischen Alter und MLQ, wie er in anderen Studien (Chang et al. 2014; Alves Nayara Silva et al. 2016) bei Personen mit kognitiver Einschränkung festgestellt wurde, konnte in dieser Studie nicht bestätigt werden. Durch die zahnmedizinische Behandlung verbesserten sich die OHIP-G14-Summenwerte der Studienpatienten auf ein mit der Allgemeinbevölkerung vergleichbares Niveau.

\subsubsection{Bewertung der MLQ durch die Selbstbefragung}

Bei 27 Patienten (51,92\%) war eine Selbstbefragung möglich. Diese Patienten anworteten bei allen drei Befragungen auf die fünf Fragen des OHIP-Ge. Nur wenige Studien beziehen Personen mit kognitiver Einschränkung in die Quantifikation ihrer MLQ ein. Bei diesen Studien waren die befragten Personen in der Lage, die 14-Fragen Version des OHIP zu beantworten, weshalb bei den bisherigen Studien von einer eher leicht kognitiv eingeschränkten Personengruppen ausgegangen werden kann (Jensen et al. 2008; Couto et al. 2018). Pörtner (2009) beschreibt, dass das Einbeziehen von Personen mit kognitiver Einschränkung in Forschungsfragen als ein wichtiger Beitrag zu deren allgemeiner Lebensqualität gewertet werden kann:

"Grundsätzlich wissen nicht wir, was für andere Menschen gut ist, sondern sie selber, auch wenn der Zugang zu diesem Wissen verschüttet sein mag. Allein schon der Versuch zu verstehen, verändert die Qualität der Beziehung". (Pörtner M: Ernstnehmen - zutrauen - verstehen: personzentrierte Haltung im Umgang mit geistig behinderten und pflegebedürttigen Menschen, S.28) 
Sozialwisschenschaftliche Forschungsansätze fordern in diesem Kontext das Einbeziehen von Personen mit kognitiver Einschränkung in Forschungsprojekte (Janz und Terfloth 2009).

Hinsichtlich der Fähigkeit zur Kommunikation zeigte die vorliegende Studie, dass trotz der kognitiven Einschränkung über die Hälfte der Patienten $(n=27)$ den OHIP-Ge beantworten konnte. Die Patienten gaben an, dass ihre MLQ zum Zeitpunkt T0 hauptsächlich durch Schmerzen reduziert war. Die angegebenen Schmerzen erscheinen plausibel im Zusammenhang mit dem zahnmedizinischen Behandlungsbedarf vor der Therapie. Es ist davon auszugehen, dass kariöse und/oder parodontal geschädigte Zähne vor der Therapie zu Schmerzen und damit zu einer reduzierten MLQ geführt haben. Auch Jensen et al. (2008) stellen bei einer Befragung von körperlich eingeschränkten Senioren eine reduzierte $M L Q$, vor allem bei bestehendem zahnmedizinischen Behandlungsbedarf, fest. Analog zu den Ergebnissen der vorliegenden Studie, war die MLQ bei den von Couto et al. (2018) befragten Personen mit (leichter) kognitiver Einschränkung bei einer reduzierten Mundgesundheit eingeschränkt.

Unbestritten ist, dass neben den zahnmedizinischen Befunden patientengruppenbezogene Faktoren den individuellen Anspruch und die Bewertung der MLQ beeinflussen (Steele et al. 2004). Menschen mit kognitiver Einschränkung leben aufgrund einer veränderten Wahrnehmung unter besonderen Existenzbedingungen. Menschen mit Behinderung unterliegen aufgrund der Behinderung anderen psychologischen und sozialen Einflussfaktoren als Menschen ohne Behinderung. Je nach Ätiologie der kognitiven Einschränkung leben diese Personen bereits unterschiedlich lange, bei angeborenen Behinderungen schon immer, unter diesen besonderen Existenzbedingungen. Die behinderungsassoziierten Lebensumstände führen wahrscheinlich zu einer ähnlichen Sozialisierung. Die Ätiologie der kognitiven Einschränkung hatte daher keinen signifikanten Einfluss auf die Bewertung der MLQ durch die Patienten selbst.

Die zahnmedizinische Therapie führte in der Patientenbefragung zu einer verbesserten MLQ. Zwischen den einzelnen Befragungen konnten einzelne Therapiemaßnahmen bzw. therapeutische Konsequenzen als signifikante Einflussfaktoren auf die Stärke der Summenwertveränderungen des OHIP-Ge verifiziert werden. Es konnten allerdings keine signifikanten Einflussfaktoren auf den 
OHIP-Ge zum Zeitpunkt T0 festgestellt werden. Ein Grund hierfür waren vermutlich die diversen Ausprägungen der kategorialen Merkmale bei relativ geringer Stichprobengröße $(n=27)$. Analog zu den Betreuungspersonen gaben nur wenige Patienten Einschränkungen in den Bereichen Ästhetik, Funktion oder soziale Akzeptanz an. Eventuell spiegelt dieses Ergebnis eine generell reduzierte Erwartungshaltung der Patienten an ihre MLQ wider.

Je mehr Zähne im Rahmen der ITN mit Füllungen versorgt wurden, desto stärker war die OHIP-Ge-Summenwertreduktion zwischen den Befragungszeitpunkten T0 und T1. Es ist wahrscheinlich, dass durch die Füllungstherapie bestehende Zahnschmerzen beseitigt wurden. Wenn Zähne aufgrund von Karies entfernt wurden, beeinflusste dies die Summenwertveränderung von T1 zu T2 eher negativ. Die Anzahl der entfernten Zähne wirkte sich außerdem negativ auf die Summenwertreduktion von T0 zu T2 aus. Je mehr Zähne gezogen wurden, desto weniger stark war die Verbesserung der MLQ. Dieses Ergebnis entspricht den Ergebnissen von Couto et al. (2018), bei denen die befragten Personen ihre MLQ bei einer geringen Zahnanzahl als eingeschränkter bewerteten. Ein Einfluss des Stützzonenverlustes auf die MLQ lies sich nicht verifizieren. Trotz Zahnlücken und reduzierter vertikaler Kieferabstützung beurteilten die Patienten ihre MLQ zu T2 signifikant besser als vor der Therapie. Die fehlende Kenntnis von Alternativen (prothetische Lückenversorgungen), die Angst vor weiteren Behandlungen und die Freude über die Beseitigung der Zahnschmerzen könnten Gründe für eine Zufriedenheitsäußerung bei objektiv suboptimalem Mundgesundheitszustand (Lückengebiss) sein. Die Ergebnisse der Selbstbefragung zeigen, dass die MLQ der Patienten vor der zahnmedizinischen Therapie vor allem durch Schmerzen reduziert war und sich nach der zahnmedizinischen Therapie verbesserte.

\subsubsection{Korrelation von Betreuer- und Patientenbefragung}

Durch die Integration der Patienten mit kognitiver Einschränkung - im Sinne eines personenzentrierten Ansatzes - ermöglicht das vorliegende Studiendesign erstmals einen Vergleich der MLQ-Einschätzungen von erwachsenen Personen mit kognitiver Einschränkung und ihren Betreuungspersonen. Fremd- 
und Selbsteinschätzung waren in der vorliegenden Studie moderat korreliert. Ein Vergleich der Einschätzungen von Betreuungsperson und Betreutem ist nur möglich, wenn der Betreute (trotz kognitiver Einschränkung) verbal kommunizieren kann. Ein Ansatzpunkt der Studie war es, den Patienten mit kognitiver Einschränkung die Kommunikation in Bezug auf ihre MLQ zu vereinfachen (Fragebogen in leichter Sprache) und innen dadurch die Teilnahme an der Quantifikation ihrer MLQ zu ermöglichen. Trotz des vereinfachten Fragebogens war aufgrund der schwer bis sehr schwer eingeschränkten Klientel nicht bei allen Patienten eine Selbstbefragung möglich. Bei Patienten ohne verbales Kommunikationsvermögen stellt die Fremdbefragung die einzige Möglichkeit zur Evaluation der MLQ dar. Es ist theoretisch vorstellbar, wie es ist, blind, taub oder gelähmt zu sein. Der Zustand der kognitiven Einschränkung ist jedoch unvorstellbar, weil dazu die imaginative Ich-Instanz selbst reduziert werden müsste (Mohr 2014). Ob die Betreuer sich in die von innen betreute Person hineinversetzen können und deren MLQ analog zu den Patienten ohne Kommunikationsvermögen selbst einschätzen, ist unklar. Wertvorstellungen und Charktereigenschaften des Fremdbefragten beeinflussen die Bewertung der MLQ (Eiser und Morse 2001; Barbosa und Gavião 2008; Sischo und Broder 2011; Lee et al. 2017). Eventuell übertragen Betreuungspersonen ihre persönlichen MLQMaßstäbe auf die Fremdbewertung. Diese Masstäbe müssen nicht identisch mit den Ansprüchen der Personen mit kognitiver Einschränkung sein. Wertende Komponenten im Zusammenhang mit der speziellen Personenklientel könnten ebenfalls die Fremdbefragung beeinflusst haben. Die OHIP-G14-Summenwerte reduzierten sich in der Gruppe, in der die Patienten zusätzlich selbst befragt werden konnten, weniger stark. Es könnte vermutet werden, dass bestehende Einschränkungen nach der Therapie von den Betreuungspersonen bei Personen ohne verbale Kommunikation eher weniger wahrgenommen wurden. In einer systematischen Übersichtsarbeit kommen Barbosa und Gavião (2008) zu dem Ergebnis, dass die Elternperspektive nicht zwangsläufig der Einschätzung der Kinder entspricht. Reissmann et al. (2017) zeigten eine moderate Korrelation $(p<0,001, r=0,38) z w i s c h e n$ den Aussagen von Kindern und ihren Eltern. Bei der Bewertung der MLQ von Jugendlichen mit Zahnstellungsanomalien schätzten die Eltern die Einschränkungen der MLQ ihrer Kinder geringer ein als die Jugendlichen selbst (Abreu et al. 2015). Jokovic et al. (2004) stellen fest, 
dass Eltern die MLQ eher übereinstimmend mit ihren Kindern einschätzen, wenn die Kinder jünger sind. Sie erklären dieses Ergebnis damit, dass jüngere Kinder mehr Zeit gemeinsam mit ihren Eltern verbringen.

Entsprechend der Studie von Jokovic et al. (2004) wurde außerdem vermutet, dass Fremd- und Selbstbefragung je eher übereinstimmen, desto mehr Zeit der Fremd- und Selbstbefragte pro Tag miteinander verbringen. Diese Vermutung konnte im Rahmen dieser Studie nicht bestätigt werden. Die Betreuungszeit betrug durchschnittlich 729,4 \pm 483,2 Minuten/Tag und hatte keinen signifikanten Einfluss auf die Übereinstimmung zwischen Patienten und Betreuern. Betreuungspersonen, die mehr Zeit pro Tag mit den Patienten verbrachten, bewerteten die Verbeserung der MLQ zu T1 allerdings höher, als die Betreuungspersonen, die weniger Zeit pro Tag mit dem Patienten verbrachten. Es ist davon auszugehen, dass Personen mit einer hohen täglichen Betreuungszeit besonders eingeschränkt sind und dass bei diesen Personen die Selbstbefragung eher weniger möglich ist, so dass sich die signifikant stärkere Verbesserung nicht auf die Korrelation zwischen Fremd- und Selbstbefragung auswirkt.

Eventuell spielt nicht die Quantität, sondern die Qualität der Betreuung eine Rolle in der Fremdeinschätzung. Der Einfluss von Eigenschaften oder subjektiven Einstellungen der Betreuungsperson auf die Fremdeinschätzung der MLQ wurde im Rahmen dieser Studie nicht überprüft. Die Übersichtsarbeit von Robertson et al. (2017) zeigt, dass sowohl die aktuelle Stimmung als auch die Erfahrung in der Pflege die Qualität der Fremdeinschätzung beeinflussen können. Es wäre denkbar, dass leichte Verhaltensänderungen im Zusammenhang mit der MLQ von den Betreuungspersonen nur bemerkt werden, wenn diese die zu betreuenden Personen gut kennen, sie schon viel Erfahrung in der Betreuung von Personen mit kognitiver Einschränkung haben oder wenn die Personen mit der Betreuungsperson auch verbal kommunizieren können. Durch die moderate Übereinstimmung, zwischen der Einschätzung der Betreuer und der Patienten, kann davon ausgegangen werden, dass die Fremdbefragung als Instrument zur Bestimmung der MLQ bei Personen mit kognitiver Einschränkung eingesetzt werden kann. 


\subsection{Fazit}

Fremd- und Selbstbefragung zeigten, dass die umfangreiche zahnärztliche Therapie in ITN die MLQ der Patienten signifikant verbessert. Eine Kombination aus Fremd- und Selbstbefragung ist zur Evaluation der MLQ bei Personen mit kognitiver Einschränkung geeignet. Trotz der Limitationen der Studie (keine Validierung des OHIP-Ge und keine Vergleichsgruppe) liefern die Ergebnisse der vorliegenden Arbeit neue Erkenntnisse zu Einflussfaktoren auf die Bewertung der $M L Q$ von Personen mit kognitiver Einschränkung. Generell Bedarf es weiterer Untersuchungen zur MLQ und zur zahnmedizinischen Versorgungssituation von Personen mit (schweren) kognitiven Einschränkungen. 


\section{Zusammenfassung}

Ziel der Studie: Ziel der Untersuchung war es, die mundgesundheitsbezogene Lebensqualität (MLQ) von Patienten mit kognitiver Einschränkung vor und nach zahnärztlicher Sanierung in Vollnarkose durch Selbst- und durch Fremdbefragung zu erfassen und patientenspezifische Einflussfaktoren auf die MLQ von Patienten mit kognitiver Einschränkung zu ermitteln.

Material und Methoden: Die MLQ von erwachsenen Patienten mit kognitiven Einschränkungen ( $n=52$ ) wurde vor der zahnärztlichen Behandlung (T0) sowie zwei bis drei Wochen (T1) und zwölf Wochen (T2) postoperativ erfasst. Dazu wurden die Betreuer der Patienten $(n=51)$ mit dem OHIP-G14 (Oral Health Impact Profile, deutsche Version, maximaler Summenwert: 56) sowie - wenn möglich - die PatientInnen selbst $(n=27)$ mit dem OHIP-Ge (dem OHIP-G5 übersetzt in leichte Sprache, maximaler Summenwert: 20) zu allen drei Zeitpunkten befragt. Zusätzlich wurden anamnestische Faktoren erhoben sowie die Mundgesundheit (DMFT, PSI, Stützzonen) vor und nach der Behandlung erfasst. Die statistische Auswertung erfolgte mit ein- und mehrfaktoriellen ordinalen Regressionen sowie Spearman-Korrelation $(p<0,05)$.

Ergebnisse: Selbst- (T0: 5,8 \pm 4,6; T1: 4,3 $\pm 5,9$; T2: $2,0 \pm 3,9$ ) und Fremdeinschätzung (T0: 18,8 $\pm 11,6$; T1: 10,1 $\pm 11,2 ; \mathrm{T} 2: 2,7 \pm 5,3$ ) zeigten eine signifikante Verbesserung der MLQ und waren moderat korreliert $(p<0,001$; $r_{S p}=0,43$ ). Die MLQ zum Zeitpunkt T0 wurde durch angeborene kognitive Einschränkungen und einen parodontalen Behandlungsbedarf (OHIP-G14) negativ beeinflusst. Die Verbesserung der MLQ war bei der Selbst- und bei der Fremdbefragung mit dem Umfang und der Art der zahnmedizinischen Therapiemaßnahmen assoziiert. Die Anzahl an Füllungen und Zahnextraktionen beeinflusste die Bewertung der MLQ durch die Patienten (OHIP-Ge). Die Ätiologie der kognitiven Einschränkung und die tägliche Betreuungszeit beeinflussten die Fremdbefragung (OHIP-G14).

Schlussfolgerungen: Die MLQ von Patienten mit kognitiver Einschränkung wird durch die zahnärztliche Behandlung in Intubationsnarkose verbessert. 


\section{$7 \quad$ Literaturverzeichnis}

Aarabi G, John MT, Schierz O, Heydecke G, Reissmann DR (2015): The course of prosthodontic patients' oral health-related quality of life over a period of 2 years. J Dent $\underline{43}, 261-268$

Abreu LG, Melgaço CA, Abreu MHNG, Lages EMB, Paiva SM (2015): Agreement between adolescents and parents/caregivers in rating the impact of malocclusion on adolescents' quality of life. Angle Orthod $\underline{85}, 806-811$

Adeyemo WL, Taiwo OA, Oderinu OH, Adeyemi MF, Ladeinde AL, Ogunlewe MO (2012): Oral health-related quality of life following non-surgical (routine) tooth extraction: A pilot study. Contemp Clin Dent $\underline{3}, 427-432$

Ainamo J, Barmes D, Beagrie G, Cutress T, Martin J, Sardo-Infirri J (1982): Development of the World Health Organization (WHO) community periodontal index of treatment needs (CPITN). Int Dent J $\underline{32}, 281-291$

Allen PF (2003): Assessment of oral health related quality of life. Health Qual Life Outcomes $\underline{1}, 40$

Allen PF, McMillan AS, Walshaw D, Locker D (1999): A comparison of the validity of generic- and disease-specific measures in the assessment of oral health-related quality of life. Community Dent Oral Epidemiol 27, 344352

Allen PF, McMillan AS, Locker D (2001): An assessment of sensitivity to change of the Oral Health Impact Profile in a clinical trial. Community Dent Oral Epidemiol 29, 175-182

Allison PJ, Locker D, Feine JS (1997): Quality of life: a dynamic construct. Soc Sci Med 45, 221-230

Alves NS, Gavina VP, Cortellazzi KL, Antunes L A A, Silveira FM, Assaf AV (2016): Analysis of clinical, demographic, socioeconomic, and psychosocial determinants of quality of life of persons with intellectual disability: a cross-sectional Study. Spec Care Dentist 36, 307-314

Ananthanarayan C, Sigal M, Godlewski W (1998): General anesthesia for the provision of dental treatment to adults with developmental disability. Anesth Prog 45, 12-17

Anders PL, Davis EL (2010): Oral health of patients with intellectual disabilities: a systematic review. Spec Care Dentist $\underline{30}, 110-117$

Arumugam A, Raja K, Venugopalan M, Chandrasekaran B, Kovanur Sampath K, Muthusamy H, Shanmugam N (2016): Down syndrome-A narrative review with a focus on anatomical features. Clin Anat $\underline{29}, 568-577$ 
Awad MA, Locker D, Korner-Bitensky N, Feine JS (2000): Measuring the effect of intra-oral implant rehabilitation on health-related quality of life in a randomized controlled clinical trial. J Dent Res $\underline{79}$, 1659-1663

Bagattoni S, Sadotti A, D'Alessandro G, Piana G (2017): Dental trauma in Italian children and adolescents with special health care needs. A crosssectional retrospective study. Eur J Paediatr Dent 18, 23-26

Barbosa TS, Gavião MBD (2008): Oral health-related quality of life in children: part III. Is there agreement between parents in rating their children's oral health-related quality of life? A systematic review. Int J Dent Hyg $\underline{6}$, 108113

Bettie NF, Ramachandiran H, Anand V, Sathiamurthy A, Sekaran P (2015): Tools for evaluating oral health and quality of life. J Pharm Bioallied Sci 7, S414-S419

Bickley SR (1978): Gingival hyperplasia in epilepsy. Br Dent Surg Assist $\underline{37}$, 104-106

Bissar A-R, Kaschke I, Schulte AG (2010): Oral health in 12- to 17-year-old athletes participating in the German Special Olympics. Int J Paediatr Dent $\underline{20}, 451-457$

Bortoluzzi MC, Traebert J, Lasta R, Da Rosa TN, Capella DL, Presta AA (2012): Tooth loss, chewing ability and quality of life. Contemp Clin Dent $\underline{3}$, 393397

Bozkurt M, Tutuncuoglu S, Serdaroglu G, Tekgul H, Aydogdu S (2004): Gastroesophageal reflux in children with cerebral palsy: efficacy of cisapride. J Child Neurol 19, 973-976

Braegger CP (1998): [Gastroesophageal reflux in infants and children]. Schweiz Med Wochenschr 128, 1822-1825

Brauchle F, Noack M, Reich E (2013): Impact of periodontal disease and periodontal therapy on oral health-related quality of life. Int Dent $\mathrm{J} \underline{63}, 306-$ 311

Bredel U, Maass C: Leichte Sprache: theoretische Grundlagen, Orientierung für die Praxis. Dudenverlag, Berlin 2016

Brennan DS, Spencer AJ, Roberts-Thomson KF (2008): Tooth loss, chewing ability and quality of life. Qual Life Res $\underline{17}$, 227-235

Bundeszahnärztekammer (BZÄK), Kassenzahnärztliche Bundesvereinigung (KZBV) Mundgesund trotz Handicap und hohem Alter, Konzept zur vertragszahnärztlichen Versorgung von Pflegebedürftigen und Menschen mit Behinderungen, Berlin 2017

Bullinger M (1997): Generic quality of life assessment in psychiatry. Potentials and limitations. Eur Psychiatry 12, 203-209 
Bullinger M (2000): Erfassung der gesundheitsbezogenen Lebensqualität mit dem SF-36-Health Survey. Bundesgesundheitsblatt Gesundheitsforschung Gesundheitsschutz $\underline{43}, 190-197$

Bullinger M (2002): Assessing health related quality of life in medicine. An overview over concepts, methods and applications in international research. Restor Neurol Neurosci 20, 93-101

Carlos JP, Gittelsohn AM (1965): Longitudinal studies of the natural history of caries-II: A life-table study of caries incidence in the permanent teeth. Arch Oral Biol 10, 739-751

Carroll JB: Human cognitive abilities: a survey of factor-analytic studies. Cambridge University Press, Cambridge; New York 1993

Chang J, Kim H-Y (2017): Prognostic factors of single-visit endodontic and restorative treatment under general anaesthesia for special needs patients. J Oral Rehabil $\underline{44}$, 96-104

Chang J, Patton LL, Kim H-Y (2014): Impact of dental treatment under general anesthesia on the oral health-related quality of life of adolescents and adults with special needs. Eur J Oral Sci $\underline{122}, 363-371$

Cheng RHW, Leung WK, Corbet EF, King NM (2007): Oral health status of adults with Down syndrome in Hong Kong. Spec Care Dentist 27, 134138

Corrêa-Faria P, Martins CC, Bönecker M, Paiva SM, Ramos-Jorge ML, Pordeus IA (2016): Clinical factors and socio-demographic characteristics associated with dental trauma in children: a systematic review and metaanalysis. Dent Traumatol $\underline{32}, 367-378$

Corson MA, Boyd T, Kind P, Allen PF, Steele JG (1999): Performance Review: Measuring oral health: does your treatment really make a difference. $\mathrm{Br}$ Dent J 187, 481-484

Couto P, Pereira PA, Nunes M, Mendes RA (2018): Oral health-related quality of life of Portuguese adults with mild intellectual disabilities. $\underline{13}$, e0193953

Cushing AM, Sheiham A, Maizels J (1986): Developing socio-dental indicators-the social impact of dental disease. Community Dent Health $\underline{3}, 3-17$

Cutando-Soriano A, Gómez-Moreno G, Bravo M (1998): Free interleukin-2 receptors in children with trisomy 21 (Down's syndrome) and different levels of periodontal disease. Int J Paediatr Dent $\underline{8}, 177-180$

Cutress TW, Hunter PB, Beck DJ, de Souza P (1978): A comparison of WHO periodontal status index with the periodontal and oral hygiene indices. Community Dent Oral Epidemiol $\underline{6}, 245-252$ 
Davis DM, Fiske J, Scott B, Radford DR (2000): The emotional effects of tooth loss: a preliminary quantitative study. Br Dent J $\underline{188}, 503-506$

Deps TD, Angelo GL, Martins CC, Paiva SM, Pordeus IA, Borges-Oliveira AC (2015): Association between Dental Caries and Down Syndrome: A Systematic Review and Meta-Analysis. PloS One $\underline{10}$, e0127484

Doege D: Bewältigungsverhalten bei Eltern von Kindern mit geistiger Behinderung: Quantitative und qualitative Untersuchung von Bewältigungsmustern. Phil. Diss. Heidelberg 2017

Dougherty N (2009): The dental patient with special needs: a review of indications for treatment under general anesthesia. Spec Care Dentist 29, 1720

Dugas NN, Lawrence HP, Teplitsky P, Friedman S (2002): Quality of life and satisfaction outcomes of endodontic treatment. J Endod 28, 819-827

Eichner K (1990): Renewed examination of the group classification of partially edentulous arches by Eichner and application advices for studies on morbidity statistics. Stomatol DDR $\underline{40}, 321-325$

Eiser C, Morse R (2001): Can parents rate their child's health-related quality of life? Results of a systematic review. Qual Life Res $\underline{10}$, 347-357

El Ashiry EA, Alaki SM, Nouri SM (2016): Oral Health Quality of Life in Children with Cerebral Palsy: Parental Perceptions. J Clin Pediatr Dent $\underline{40}$, 375387

FDI: FDI's definition of oral health. https://www.fdiworlddental.org/oralhealth/fdis-definition-of-oral-health; Zugriff am 09.03.2018

Fernandez C, Kaschke I, Perlman S, Koehler B, Marks L (2015): A multicenter study on dental trauma in permanent incisors among Special Olympics athletes in Europe and Eurasia. Clin Oral Investig 2, 135-141

Ferreira MC, Dias-Pereira AC, Branco-de-Almeida LS, Martins CC, Paiva SM (2017): Impact of periodontal disease on quality of life: a systematic review. J Periodontal Res $\underline{52}$, 651-665

Festa SA, Ferguson FS, Hauk M (1993): Behavior management techniques in pediatric dentistry. N Y State Dent J $\underline{59}, 35-38$

Fischer KR, Lindner I, Fickl S (2015): Implant treatment in periodontally compromised subjects-quality of life and patient satisfaction. Clin Oral Investig 20, 697-702

Frydman A, Nowzari H (2012): Down syndrome-associated periodontitis: a critical review of the literature. Compend Contin Educ Dent 33, 356-361

Gabre P (2000): Studies on oral health in mentally retarded adults. Swed Dent J Suppl $\underline{142} 1-48$ 
Gerritsen AE, Allen PF, Witter DJ, Bronkhorst EM, Creugers NHJ (2010): Tooth loss and oral health-related quality of life: a systematic review and metaanalysis. Health Qual Life Outcomes $\underline{8}, 126$

Glassman P (2009): A review of guidelines for sedation, anesthesia and alternative interventions for people with special needs. Spec Care Dentist 29, 9 16

Glassman P, Caputo A, Dougherty N, Lyons R, Messieha Z, Miller C, Peltier B, Romer M (2009): Special Care Dentistry Association consensus statement on sedation, anesthesia, and alternative techniques for people with special needs. Spec Care Dentist 29, 2-8

Gordon SM, Dionne RA, Snyder J (1998): Dental fear and anxiety as a barrier to accessing oral health care among patients with special health care needs. Spec Care Dentist 18, 88-92

Groß D: Ethik in der Zahnmedizin. Quintessenz Verlag, Berlin 2012

Hassel AJ, Rolko C, Leisen J, Schmitter M, Rexroth W, Leckel M (2007): Oral health-related quality of life and somatization in the elderly. Qual Life Res $\underline{16}, 253-261$

Hassel AJ, Rolko C, Koke U, Leisen J, Rammelsberg P (2008): A German version of the GOHAI. Community Dent Oral Epidemiol 36, 34-42

Hellwege K-D: Die Praxis der zahnmedizinischen Prophylaxe: ein Leitfaden für die Individualprophylaxe, Gruppenprophylaxe und initiale Parodontaltherapie. Georg Thieme Verlag, Stuttgart 2003

Hempel E, Limberger K, Möller M, Heinrich-Weltzien R (2015): Oral health status of students with and without disabilities in Erfurt, Germany. Gesundheitswesen $\underline{77}$, 263-268

Heydecke G, Locker D, Awad MA, Lund JP, Feine JS (2003): Oral and general health-related quality of life with conventional and implant dentures. Community Dent Oral Epidemiol 31, 161-168

Hirschberg M: Behinderung im internationalen Diskurs. Die flexible Klassifizierung der Weltgesundheitsorganisation. Rehabil $\underline{53}$, 424-424 Campus, Frankfurt 2014

Ho HD, Satur J, Meldrum R (2017): Perceptions of oral health by those living with mental illnesses in the Victorian Community - The consumer's perspective. Int J Dent Hyg $\underline{16}, 10-16$

Idaira Y, Nomura Y, Tamaki Y, Katsumura S, Kodama S, Kurata K, Asada Y (2008): Factors affecting the oral condition of patients with severe motor and intellectual disabilities. Oral Dis $\underline{14}, 435-439$

Jaber MA (2011): Dental caries experience, oral health status and treatment needs of dental patients with autism. J Appl Oral Sci 19, 212-217 
Jang E-J, Kim E-K, Lee K-S, Lee H-K, Choi Y-H, Hwang T-Y, Kim SK, Jo MW (2015): Oral health related quality of life and it's related factors of stroke patients at home in Korea. Arch Gerontol Geriatr $\underline{61}, 523-528$

Janz F, Terfloth K: Forschung im Kontext geistiger Behinderung. In: Janz F, Terfloth $\mathrm{K}$ (Hrsg.): Empirische Forschung im Kontext geistiger Behinderung. Edition S; Winter, Heidelberg 2009, 9-16

Jenei Á, Sándor J, Hegedűs C, Bágyi K, Nagy L, Kiss C, Szabó G, Márton IJ (2015): Oral health-related quality of life after prosthetic rehabilitation: a longitudinal study with the OHIP questionnaire. Health Qual Life Outcomes $\underline{13,98-99}$

Jensen PM, Saunders RL, Thierer T, Friedman B (2008): Factors associated with oral health-related quality of life in community-dwelling elderly persons with disabilities. J Am Geriatr Soc $\underline{56}, 711-717$

John MT (2004): Normwerte mundgesundheitsbezogener Lebensqualität für Kurzversionen des Oral Health Impact Profile. Schweiz Monatsschr Zahnmed 114, 784-781

John MT (2007): Exploring dimensions of oral health-related quality of life using experts' opinions. Qual Life Res $\underline{16}, 697-704$

John MT, Patrick DL, Slade GD (2002): The German version of the Oral Health Impact Profile - translation and psychometric properties. Eur J Oral Sci $\underline{110}, 425-433$

John MT, LeResche L, Koepsell TD, Hujoel P, Miglioretti DL, Micheelis W (2003): Oral health-related quality of life in Germany. Eur J Oral Sci 111, 483-491

John MT, Hujoel P, Miglioretti DL, LeResche L, Koepsell TD, Micheelis W (2004): Dimensions of oral-health-related quality of life. J Dent Res $\underline{83}$, 956-960

John MT, Reissmann DR, Feuerstahler L, Waller N, Baba K, Larsson P, Celebić A, Szabo G, Rener-Sitar K (2014): Exploratory factor analysis of the Oral Health Impact Profile. J Oral Rehabil 41, 635-643

John MT, Reissmann DR, Čelebić A, Baba K, Kende D, Larsson P, Rener-Sitar $K$ (2016): Integration of oral health-related quality of life instruments. J Dent 53, 38-43

John MT, Michaeelis W: Mundgesundheitsbezogene Lebensqualität in der Bevölkerung: Grundlagen und Ergebnisse des Oral Health Impact Profile $(\mathrm{OHIP})$ aus einer repräsentativen Stichprobe in Deutschland. IDZ, Köln 2003

Jokovic A, Locker D, Guyatt G (2004): How well do parents know their children? implications for proxy reporting of child health-related quality of life. Qual Life Res $\underline{13}$, 1297-1307 
Jordan AR, Micheelis W, Institut der Deutschen Zahnärzte (Hrsg.): Fünfte Deutsche Mundgesundheitsstudie (DMS V). Deutscher Zahnärzte Verlag DÄV, Köln 2016

Kane RA, Kling KC, Bershadsky B, Kane RL, Giles K, Degenholtz HB, Liu J, Cutler LJ (2003): Quality of Life Measures for Nursing Home Residents. J Gerontol Ser A $\underline{58}, 240-248$

Kaschke I (2008): Mundgesundheitsförderung für erwachsene Bewohner von Behinderteneinrichtungen-Evaluation des Berliner Modellprojektes Masterarbeit Studiengang Psychosoziale Prävention und Gesundheitsförderung. FU Berlin

Kiliaridis S (1995): Masticatory muscle influence on craniofacial growth. Acta Odontol Scand $\underline{53}, 196-202$

Klein H, Palmer CE, Knutson JW (1938): Studies on Dental Caries: I. Dental Status and Dental Needs of Elementary School Children. Public Health Rep $\underline{53}, 751-765$

Kolenda J, Fischer-Brandies H, Ciesielski R, Koos B (2016): Oral health-related quality of life after orthodontic treatment for anterior tooth alignment: Association with emotional state and sociodemographic factors. J Orofac Orthop $\underline{77}, 138-145$

Kothari M, Pillai RS, Kothari SF, Spin-Neto R, Kumar A, Nielsen JF (2017): Oral health status in patients with acquired brain injury: a systematic review. Oral Surg Oral Med Oral Pathol Oral Radiol 123, 205-219.e7

Kramer F-J, Gruber R, Fialka F, Sinikovic B, Schliephake H (2008): Quality of Life and Family Functioning in Children with Nonsyndromic Orofacial Clefts at Preschool Ages. J Craniofac Surg $\underline{19}, 580$

Lee Y-C, Yang H-J, Lee W-T, Teng M-J (2017): Do parents and children agree on rating a child's HRQOL? A systematic review and Meta-analysis of comparisons between children with attention deficit hyperactivity disorder and children with typical development using the PedsQLTM. Disabil Rehabil 41, 265-275

Leroy R, Declerck D (2013): Objective and subjective oral health care needs among adults with various disabilities. Clin Oral Investig 17, 1869-1878

Lim MAWT, Borromeo GL (2017): The use of general anesthesia to facilitate dental treatment in adult patients with special needs. J Dent Anesth Pain Med 17, 91-103

Limeres Posse J, Vázquez García E, Medina Henríquez J, Tomás Carmona I, Fernández Feijoo J, Diz Dios P (2003): Pre-assessment of severely handicapped patients suitable of dental treatment under general anesthesia. Med Oral $\underline{8}, 353-360$ 
Locker D (1988): Measuring oral health: a conceptual framework. Community Dent Health $\underline{5}, 3-18$

Locker D, Slade G (1994): Association between clinical and subjective indicators of oral health status in an older adult population. Gerodontology $\underline{11}$, 108-114

Locker D, Gibson B (2006): The concept of positive health: a review and commentary on its application in oral health research. Community Dent Oral Epidemiol 34, 161-173

Locker D, Jokovic A, Clarke M (2004): Assessing the responsiveness of measures of oral health-related quality of life. Community Dent Oral Epidemiol $\underline{32}, 10-18$

Loureiro AC, Costa FO, Costa JE (1990): The oral health in different groups of adults with mental handicaps attending Birmingham (UK) adult training centres. Community Dent Health $\underline{7}, 135-141$

Lyons RA (2009): Understanding basic behavioral support techniques as an alternative to sedation and anesthesia. Spec Care Dentist 29, 39-50

Mallineni SK, Yiu CKY (2015): Dental treatment under general anesthesia for special-needs patients: analysis of the literature. J Investig Clin Dent $\underline{7}$, 325-331

Malter S, Hirsch C, Reissmann DR, Schierz O, Bekes K (2015): Effects of method of administration on oral health-related quality of life assessment using the Child Perceptions Questionnaire (CPQ-G11-14). Clin Oral Investig $\underline{19}, 1939-1945$

Manley MC, Skelly AM, Hamilton AG (2000): Dental treatment for people with challenging behaviour: general anaesthesia or sedation? $\mathrm{Br}$ Dent $\mathrm{J} \underline{188}$, 358-360

Manton KG (1982): Changing concepts of morbidity and mortality in the elderly population. Milbank Mem Fund Q Health Soc $\underline{60}$, 183-244

Marks L, Fernandez C, Kaschke I, Perlman S (2015): Oral cleanliness and gingival health among Special Olympics athletes in Europe and Eurasia. Med Oral Patol Oral Cir Bucal 20, 591-597

Matevosyan NR (2010): Oral Health of Adults with Serious Mental Illnesses: A Review. Community Ment Health J $\underline{46}, 553-562$

Mayer KU: Zukunft leben: Die demografische Chance. Nicolaiverlag, Berlin 2013

Mazzoleni S, Drigo P, Da Dalt L, Battistella PA, Casara GL, Donzelli F, Zacchello $F$ (1991): [Gastroesophageal reflux and infantile cerebral palsy: our experience and review of the literature]. Pediatr Med Chir 13, 293-296 
McGrath C, McMillan AS, Zhu HW, Li LSW (2009): Agreement between patient and proxy assessments of oral health-related quality of life after stroke: an observational longitudinal study. J Oral Rehabil 36, 264-270

Mendez M, Angst PM, Stadler AF, Oppermann RV, Gomes S (2017): Impacts of supragingival and subgingival periodontal treatments on oral healthrelated quality of life. Int J Dent Hyg $\underline{15}, 135-141$

Messieha Z, Ananda RC, Hoffman I, Hoffman W (2007): Five Year Outcomes Study of Dental Rehabilitation Conducted Under General Anesthesia for Special Needs Patients. Anesth Prog $\underline{54}, 170-174$

Meyer J, Belser U, Hotz P, Lüthy H, Baehni P (2004): Forschung · Wissenschaft Recherche - Science. Schweiz Monatsschr Zahnmed 114, 9

Mizutani S, Ekuni D, Tomofuji T, Azuma T, Kataoka K, Yamane M, Iwasaki Y, Morita M (2015): Relationship between xerostomia and gingival condition in young adults. J Periodontal Res $\underline{50}, 74-79$

Mohr L: Schwerste Behinderung und theologische Anthropologie. ATHENAVerlag, Oberhausen 2014

Molina-García A, Castellanos-Cosano L, Machuca-Portillo G, Posada-de la Paz M (2016): Impact of rare diseases in oral health. Med Oral Patol Oral Cir Bucal 21, e587-594

Mühlberg S, Jäger J, Krohn-Grimberghe B, Patschan S, Mausberg RF, Schmalz G, Haak R, Ziebolz D (2017): Oral health-related quality of life depending on oral health in patients with rheumatoid arthritis. Clin Oral Investig 114, 784-791

Müller F, Nitschke I: Der alte Patient in der zahnärztlichen Praxis. Quintessenz Verlag, Berlin 2010

Naito M, Yuasa H, Nomura Y, Nakayama T, Hamajima N, Hanada N (2006): Oral health status and health-related quality of life: a systematic review. J Oral Sci $\underline{48}, 1-7$

Naseem M, Shah AH, Khiyani MF, Khurshid Z, Zafar MS, Gulzar S, AlJameel $\mathrm{AH}$, Khalil HS (2016): Access to oral health care services among adults with learning disabilities: a scoping review. Ann Stomatol (Roma) $\underline{7}, 52-$ 59

Neuhäuser G: Syndrome bei Menschen mit geistiger Behinderung: Ursachen, Erscheinungsformen und Folgen. 3. erw. und überarb. Aufl; LebenshilfeVerlag, Marburg 2010

Nickenig H-J, Wichmann M, Andreas SK, Eitner S (2008): Oral health-related quality of life in partially edentulous patients: assessments before and after implant therapy. J craniomaxillofac Surg $\underline{36}, 477-480$ 
Nitschke I, Hopfenmüller W (1991): Dental care in homes for the elderly and aged. Organization and opinion of home management. Dtsch Stomatol Berl Ger $\underline{41}$, 432-435

Nitschke I, Kaschke I (2011): Special care dentistry for dependent elderly and people with disabilities. Bundesgesundheitsblatt Gesundheitsforschung Gesundheitsschutz $\underline{54}, 1073-1082$

Nowossadeck S (2013): Demografischer Wandel, Pflegebedürftige und der künftige Bedarf an Pflegekräften. Bundesgesundheitsblatt Gesundheitsforschung Gesundheitsschutz $\underline{56}, 1040-1047$

Nunn JH, Davidson G, Gordon PH, Storrs J (1995): A retrospective review of a service to provide comprehensive dental care under general anesthesia. Spec Care Dentist 15, 97-101

Osoba D, Zee B, Pater J, Warr D, Kaizer L, Latreille J (1994): Psychometric properties and responsiveness of the EORTC quality of Life Questionnaire (QLQ-C30) in patients with breast, ovarian and lung cancer. Qual Life Res $\underline{3}, 353-364$

Petersen PE (1990): Social inequalities in dental health. Community Dent Oral Epidemiol 18, 153-158

Pezzementi ML, Fisher MA (2005): Oral health status of people with intellectual disabilities in the southeastern United States. J Am Dent Assoc 136, 903-912

Pieper K, Dirks B, Kessler P (1986): Caries, oral hygiene and periodontal disease in handicapped adults. Community Dent Oral Epidemiol 14, 28-30

Pinel JPJ, Pauli P: Biopsychologie. 8. Auflage; Pearson, München 2012

Pohl Y, Filippi A, Geiger G, Kirschner H, Boll M (1996): Dental treatment of handicapped patients using endotracheal anesthesia. Anesth Prog $\underline{43}$, 20-23

Pommerenke F, Schumacher GH, Becker R, Hübner A (1988): The tongue as a factor in craniofacial growth. 4. Results of animal experiments. Anat Anz $\underline{167}, 281-287$

Pörtner M: Ernstnehmen - zutrauen - verstehen: personzentrierte Haltung im Umgang mit geistig behinderten und pflegebedürftigen Menschen. (Konzepte der Humanwissenschaften), 5. Auflage; Klett-Cotta, Stuttgart 2006

Reissmann DR, John MT, Sagheri D, Sierwald I (2017): Diagnostic accuracy of parents' ratings of their child's oral health-related quality of life. Qual Life Res $\underline{26}, 881-891$ 
Robertson S, Cooper C, Hoe J, Hamilton O, Stringer A, Livingston G (2017): Proxy rated quality of life of care home residents with dementia: a systematic review. Int Psychogeriatr 29, 569-581

Rodríguez Peinado N, Mourelle Martínez MR, Diéguez Pérez M, De Nova García MJ (2018): A study of the dental treatment needs of special patients: cerebral paralysis and Down syndrome. Eur J Paediatr Dent 19, 233-238

Rud B, Kisling E (1973): The influence of mental development on children's acceptance of dental treatment. Scand J Dent Res $\underline{81}$, 343-352

Scalioni FAR, Carrada CF, Martins CC, Ribeiro RA, Paiva SM (2018): Periodontal disease in patients with Down syndrome: A systematic review. J Am Dent Assoc 149, 628-639.e11

Schmalz G, Wendorff H, Marcinkowski A, Weinreich G, Teschler H, Haak R, Sommerwerck U, Ziebolz D (2017): Oral health related quality of life depending on oral health and specific factors in patients after lung transplantation. Clin Respir J 114, 731-737

Schmidt A, Ciesielski R, Orthuber W, Koos B (2013): Survey of oral healthrelated quality of life among skeletal malocclusion patients following orthodontic treatment and orthognathic surgery. J Orofac Orthop $\underline{74}$, 287294

Schopf P: Curriculum Kieferorthopädie. 4. überarbeitete Auflage; QuintessenzVerlag, Berlin 2008

Schulte AG, Kaschke I, Bissar A (2011): Mundgesundheit erwachsener Athleten mit geistiger Behinderung. Gesundheitswesen $\underline{73}$, e78-e83

Schulte AG, Freyer K, Bissar A (2013): Caries experience and treatment need in adults with intellectual disabilities in two German regions. Community Dent Health $\underline{30}$, 39-44

Schweyen R, Kuhnt T, Wienke A, Eckert A, Hey J (2017): The impact of oral rehabilitation on oral health-related quality of life in patients receiving radiotherapy for the treatment of head and neck cancer. Clin Oral Investig $\underline{21}, 1123-1130$

Shaw MJ, Shaw L, Foster TD (1990): The oral health in different groups of adults with mental handicaps attending Birmingham (UK) adult training centres. Community Dent Health $\underline{7}, 135-141$

Sheiham A, Nicolau B (2005): Evaluation of social and psychological factors in periodontal disease. Periodontol 2000 39, 118-131

Sierwald I, John MT, Durham J, Mirzakhanian C, Reissmann DR (2011): Validation of the response format of the Oral Health Impact Profile. Eur J Oral Sci 119 , 489-496 
Sischo L, Broder HL (2011): Oral Health-related Quality of Life. J Dent Res $\underline{90}$, 1264-1270

Sisson KL (2007): Theoretical explanations for social inequalities in oral health. Community Dent Oral Epidemiol 35, 81-88

Slade GD (1997): Derivation and validation of a short-form oral health impact profile. Community Dent Oral Epidemiol 25, 284-290

Slade GD, Spencer AJ (1994): Development and evaluation of the Oral Health Impact Profile. Community Dent Health 11, 3-11

Slade GD, Spencer AJ, Locker D, Hunt RJ, Strauss RP, Beck JD (1996): Variations in the social impact of oral conditions among older adults in South Australia, Ontario, and North Carolina. J Dent Res $\underline{75}$, 1439-1450

Slade M (2002): Routine outcome assessment in mental health services. Psychol Med $\underline{32}, 1339-1343$

Sousa KH, Kwok O-M (2006): Putting Wilson and Cleary to the test: analysis of a HRQOL conceptual model using structural equation modeling. Qual Life Res $\underline{15}$, 725-737

Steele JG, Sanders AE, Slade GD, Allen PF, Lahti S, Nuttall N, Spencer AJ (2004): How do age and tooth loss affect oral health impacts and quality of life? A study comparing two national samples. Community Dent Oral Epidemiol 32, 107-114

Stelzle F, Rohde M, Oetter N, Krug K, Riemann M, Adler W, Neukam FW, Knipfer C (2017): Gingival esthetics and oral health-related quality of life in patients with cleft lip and palate. Int J Oral Maxillofac Surg $\underline{46}$, 993-999

Strassburger C, Heydecke G, Kerschbaum T (2004): Influence of prosthetic and implant therapy on satisfaction and quality of life: a systematic literature review. Part 1--Characteristics of the studies. Int J Prosthodont 17, 8393

Szentpétery A, Szabó G, Marada G, Szántó I, John MT (2006): The Hungarian version of the Oral Health Impact Profile. Eur J Oral Sci 114, 197-203

Thilander B (1995): Basic mechanisms in craniofacial growth. Acta Odontol Scand $\underline{53}, 144-151$

Thomson WM, Mejia GC, Broadbent JM, Poulton R (2012): Construct Validity of Locker's Global Oral Health Item. J Dent Res $\underline{91}, 1038-1042$

Tiller S, Wilson KI, Gallagher JE (2001): Oral health status and dental service use of adults with learning disabilities living in residential institutions and in the community. Community Dent Health $\underline{18}, 167-171$

Torales J, Barrios I, González I (2017): Oral and dental health issues in people with mental disorders. Medwave $\underline{17}$, e7045 
UN-Behindertenrechtskonvention: Übereinkommen über die Rechte von Menschen mit Behinderungen.

https://www.behindertenrechtskonvention.info/; Zugriff am 23.07.2018

Van Bost G, Van Damme S, Crombez G (2017): The role of acceptance and values in quality of life in patients with an acquired brain injury: a questionnaire study. PeerJ $\underline{5}$, e3545

Vandenplas Y, Hegar B (2000): Diagnosis and treatment of gastro-oesophageal reflux disease in infants and children. J Gastroenterol Hepatol 15, 593603

Verbrugge LM, Jette AM (1994): The disablement process. Soc Sci Med $\underline{38}, 1-$ 14

Vellappally S, Gardens SJ, Al Kheraif A-AA, Krishna M, Babu S, Hashem M, Jacob V, Anil S (2014): The prevalence of malocclusion and its association with dental caries among 12-18-year-old disabled adolescents. BMC Oral Health $\underline{14}, 123$

Waller N, John MT, Feuerstahler L, Baba K, Larsson P, Peršić S, Kende D, Reißmann DR, Rener-Sitar K (2016): A 7-day recall period for a clinical application of the oral health impact profile questionnaire. Clin Oral Investig $\underline{20}, 91-99$

Wang Y-C, Lin I-H, Huang C-H, Fan S-Z (2012): Dental anesthesia for patients with special needs. Acta Anaesthesiol Taiwan $\underline{50}, 122-125$

Wilson IB, Cleary PD (1995): Linking clinical variables with health-related quality of life. A conceptual model of patient outcomes. JAMA 273, 59-65

Wilson-Genderson M, Broder HL, Phillips C (2007): Concordance between caregiver and child reports of children's oral health-related quality of life. Community Dent Oral Epidemiol 35, 32-40

Winter K, Baccaglini L, Tomar S (2008): A review of malocclusion among individuals with mental and physical disabilities. Spec Care Dentist $\underline{28}, 19$ 26

Winter L: Barrierefreie Kommunikation: Leichte Sprache und Teilhabe für Menschen mit Lernschwierigkeiten. Diplomica Verlag, Hamburg 2014

Wolf HF, Rateitschak EM, Rateitschak KH: Parodontologie. 3. Auflage; Georg Thieme Verlag, Stuttgart 2004

WHO, World Health Organization quality of life assessment (1995): Position paper from the World Health Organization. Soc Sci Med 41, 1403-1409

WHO, World Bank: World report on disability. WHO Geneva, 2011. http://www.who.int/disabilities/world_report/2011/report/en/; Zugriff am 07.09.2018 
Wöstmann B, Simon T, Neuhäuser-Berthold M, Rehmann P (2016): Pilot Study on the Influence of Nutritional Counselling and Implant Therapy on the Nutritional Status in Dentally Compromised Patients. PloS One 11, e0147193

Zhu HW, McGrath C, McMillan AS, Li LSW (2008): Can caregivers be used in assessing oral health-related quality of life among patients hospitalized for acute medical conditions? Community Dent Oral Epidemiol $\underline{36}, 27-33$ 


\section{Danksagung}

Ich bedanke mich bei allen Personen, die an den Befragungen zur Mundgesundheitsbezogenen Lebensqualität teilgenommen haben und mir immer wieder neue Einblicke in die Lebensrealitäten von Personen mit kognitiver Einschränkung ermöglicht haben. Mein herzlichster Dank gilt Prof. Dr. Annette Wiegand, für die fokussierte Betreuung dieses komplexen Themas. Vielen Dank für die wissenschaftliche Unterstützung und die stets freundlichen, schnellen und zielführenden Hilfestellungen während der gesamten Bearbeitungsphase meiner Dissertation. Ebenfalls bedanke ich mich bei den Mitarbeitern der Poliklinik für Präventive Zahnmedizin, Parodontologie und Kariologie im Zentrum Zahn-, Mund- und Kieferheilkunde der Universitätsmedizin Göttingen, für die Unterstützungen während der Datenerhebung.

Besonderer Dank gilt Dr. Wilhelm Bomfleur und Dr. Imke Kaschke, die mich bereits vor Beginn meiner Dissertation mit ihrem Einsatz für die zahnmedizinische Betreuung von Menschen mit Behinderung beeindruckt und geprägt haben. 